\title{
The kinetic limit of a system of coagulating Brownian particles
}

\author{
Alan Hammond ${ }^{1}$ \\ Department of Statistics \\ University of California \\ Berkeley, California 94720 \\ Fraydoun ReZakhanlod 1 \\ Department of Mathematics \\ University of California \\ Berkeley, California 94720-3840
}

\section{INTRODUCTION}

Understanding the evolution in time of macroscopic quantities such as pressure or temperature is a central task in non-equilibrium statistical mechanics. We study this problem rigorously for a model of mass-bearing Brownian particles that are prone to coagulate when they are close, where the macroscopic quantity in this case is the density of particles of a given mass. Brownian motion arises in the particles of a colloid, due to the random agitation of the much smaller molecules that form the ambient environment. As such, our model could be considered as one of a colloid, where the dominant interaction between particles is that of coagulation. A theoretical discussion of coagulation in colloids was undertaken by Smoluchowski in [8].

In the model that we consider, a large number $N$ of particles, each carrying some integer-valued mass, are, at some initial time, scattered in $\mathbb{R}^{d}$, whose dimension $d$ satisfies $d \geq 3$ for the purposes of this paper. These particles then perform Brownian motions. There is an $N$-dependent parameter $\epsilon$ that specifies the range of interaction of any particle in the model: a pair of particles is liable to coagulate (to form a new particle that combines the mass of the old two) when the distance between the two is of order $\epsilon$. The choice of $\epsilon$ as a function of $N$ is dictated by insisting that the so-called mean free path is bounded away from zero and infinity in the limit $N \rightarrow \infty$ of high particle number. (The mean free path is the mean time until the first collision of a particle drawn uniformly at random at the initial time. A scaling that produces a bounded mean free path is called a kinetic limit.) Our model incorporates a significant degree of physical realism, absent from earlier work on this type of problem, in the sense that we permit the diffusion rate of the particle to depend on its mass, including the case where this rate is taken to be decreasing in the mass (it is physically reasonable to suppose that the diffusion rate of a Brownian particle is inversely proportional to the mass). As we will later describe in precise terms, we also introduce a parameter into the mechanism of reaction which allows us to study such reactions over a natural range of their strengths.

We study the macroscopic evolution of this particle system by measuring the density of particles of a given mass $m$ in the vicinity of a macroscopic location $x$ and at some time $t$. We will prove

\footnotetext{
${ }^{1}$ Research supported in part by NSF grant DMS0307021
} 
that, when the initial number of particles is chosen to be high, this density typically evolves as the solution of the Smoluchowski system of PDE,

$$
\frac{\partial f_{n}}{\partial t}(x, t)=d(n) \Delta f_{n}(x, t)+Q_{1}^{n}(f)(x, t)-Q_{2}^{n}(f)(x, t) \quad n=1,2, \ldots
$$

with initial data $f_{n}(\cdot, 0)=h_{n}(\cdot)$, to be specified in more detail shortly. The first term on the right-hand-side of (1.1) corresponds to the diffusion among particles of mass $n$, with $d(n)$ being one-half of the diffusion rate of such particles. The terms in (1.1) corresponding to the interaction of pairs of particles are given by the gain term

$$
Q_{1}^{n}(f)(x, t)=\frac{1}{2} \sum_{m=1}^{n} \beta(m, n-m) f_{m}(x, t) f_{n-m}(x, t),
$$

and the loss term

$$
Q_{2}^{n}(f)=f_{n}(x, t) \sum_{m=1}^{\infty} \beta(m, n) f_{m}(x, t) .
$$

Here, the collection of constants $\beta: \mathbb{N}^{2} \rightarrow(0, \infty)$ quantify the macroscopic propensity of mass at a pair of values to combine. As well as deriving the system (1.1) as the typical macroscopic profile of our random model, we prove in this paper the precise relation between the macroscopic constants $\beta$ and the microscopic mechanism of reaction.

We will be concerned with weak solutions of the system (1.1), defined by the equality of the left- and right-hand sides of (1.1) after multiplication by $J_{n}: \mathbb{R}^{d} \times[0, \infty) \rightarrow[0, \infty)$, integration in space-time, and integration by parts. The equality is demanded over all choices of sequences of compactly supported smooth functions $\left\{J_{n}: n \in \mathbb{N}\right\}$ such that only finitely many terms in the sequence are not identically zero.

We now give a precise definition of the microscopic process. We in fact define a sequence of such models, indexed according to the initial number $N$ of particles in them. We define a range of interaction, $\epsilon$, according to $N \epsilon^{d-2}=Z$, where the exact value of the positive constant $Z$ will shortly be given. (We will explain heuristically at the beginning of Section 2 why this relation between $N$ and $\epsilon$ determines the regime of bounded mean free path). In defining the model, the main elements to describe are the initial random choice of particle locations and masses, the diffusive dynamics, and the mechanism for coagulation.

To describe each of these, we require notation for labelling the particles in this time-dependent model. Let a countable set $I$ of symbols be given. A configuration $q$ of particles is an $\mathbb{R}^{d} \times \mathbb{N}$-valued function on a finite subset $I_{q}$ of $I$. For any $i \in I_{q}, q(i)$ may be written as $\left(x_{i}, m_{i}\right)$. The particle labelled by $i$ has mass $m_{i}$ and location $x_{i}$. In practice, the index set $I_{q}$ will be a function of time, with a change occurring only at collision events, of which there are finitely many in any given sample of one of the random models.

As for the dynamics of the process, the action on $F$ of the infinitesmal generator $\mathbb{L}$ is given by

$$
(\mathbb{L} F)(q)=\mathbb{A}_{0} F(q)+\mathbb{A}_{C} F(q),
$$


where $F:\left\{\mathbb{R}^{d} \times \mathbb{N}\right\}^{I} \rightarrow[0, \infty)$ denotes a smooth function, its domain being given the product topology. In (1.4), the diffusion operator $\mathbb{A}_{0}$ is given by

$$
\mathbb{A}_{0} F(q)=\sum_{i \in I_{q}} d\left(m_{i}\right) \Delta_{x_{i}} F
$$

while the collision operator $\mathbb{A}_{C}$ is specified by

$$
\begin{array}{rl}
\mathbb{A}_{C} F(q)=\frac{1}{2} \sum_{i, j \in I_{q}} \epsilon^{-2} & V\left(\frac{x_{i}-x_{j}}{\epsilon}\right) \alpha\left(m_{i}, m_{j}\right) \\
\times & {\left[\frac{m_{i}}{m_{i}+m_{j}} F\left(S_{i, j}^{1} q\right)+\frac{m_{j}}{m_{i}+m_{j}} F\left(S_{i, j}^{2} q\right)-F(q)\right] .}
\end{array}
$$

Here,

- the collection of constants $\alpha: \mathbb{N}^{2} \rightarrow[0, \infty)$ are the parameters of strength of interaction between pairs of particles of given integer mass, to which we earlier alluded.

- the function $V: \mathbb{R}^{d} \rightarrow[0, \infty)$ is assumed to be continuous, of compact support, and with $\int_{\mathbb{R}^{d}} V(x) d x=1$. Its role is to include among the models we consider a rule for coagulation time that may be rather arbitrary, beyond the insistence that it be Markovian and cause reaction of a pair of particles at some time when this pair are to be found within an order of the range of interaction $\epsilon$.

- we denote by $S_{i, j}^{1} q$ that configuration formed from $q$ by removing the indices $i$ and $j$ from $I_{q}$, and adding a new index from $I$ to which $S_{i, j}^{1} q$ assigns the value $\left(x_{i}, m_{i}+m_{j}\right)$. The configuration $S_{i, j}^{2} q$ is defined in the same way, except that it assigns the value $\left(x_{j}, m_{i}+m_{j}\right)$ to the new index. The specifics of the collision event then are that the new particle appears in one of the locations of the two particles being removed, with the choice being made randomly with weights proportional to the mass of the two colliding particles.

While this choice of new particle location causes a pleasing cancellation of terms at numerous times in our proof, it is inessential: the same of method of proof yields our main theorem for any choice of location for a new particle that is microscopically close - that is, within an order of $\epsilon$ - from the place where the two old particles reacted.

We explain further our reasons for choosing the form of the collision term in (1.6), and the interpretation of the various terms. Suppose that two particles $\left(x_{i}, m_{i}\right)$ and $\left(x_{j}, m_{j}\right)$ are such that $x_{i}-x_{j}$ enters the support of $V_{\epsilon}(\cdot):=\epsilon^{-2} V\left(\frac{\dot{\epsilon}}{\epsilon}\right)$ at time $t_{0}$. They are likely to remain at a displacement of order $\epsilon$ for a time period of order $\epsilon^{2}$. Given that the dimension $d$ is at least three, it is likely that after a time that is large compared to $\epsilon^{2}$, the particles have moved apart to a greater distance, and that they will not return to an $\epsilon$-vicinity of each other at any later time. This means that the integral

$$
I_{T}=\int_{t=t_{0}}^{T} \alpha\left(m_{i}, m_{j}\right) V_{\epsilon}\left(x_{i}-x_{j}\right) d t
$$

whose derivative in $T$ is the infinitesimal rate at time $T$ for coagulation between the pair, and which increases as $T$ rises, is likely to have gained its eventual value as a function of $T$ when $T$ is of the order of $t_{0}+C \epsilon^{2}$. Thus, the normalisation by $\epsilon^{-2}$ that appears in (1.6) ensures that the integral $I_{T}$ reaches an eventual value that is of unit order. Neglecting the effects of other particles, 
the probability of collision between the pair before time $T$ is equal to $1-\exp \left\{-I_{T}\right\}$. We are thus prescribing a dynamics in which the fraction of instances of pairs of particles of displacement of order $\epsilon$ that result in a coagulation of that pair is bounded away from 0 and 1, uniformly in small values of $\epsilon$. The role of the constants $\alpha$ is to determine whether this fraction is high or not. The reason then for introducing the constants $\alpha$ is that they range over the right scale to determine precisely how liable mass-pairs are to react microscopically. The choice of the value of these constants transmits to the macroscopic reaction propensities appearing in the interaction terms (1.2) and (1.3) of the Smoluchowski PDE. The recipe for determining $\beta$ from $\alpha$ is as follows: there exists a solution $u=u_{n, m}: \mathbb{R}^{d} \rightarrow(0, \infty)$ of the equation

$$
\Delta u_{n, m}(x)=\frac{\alpha(n, m)}{d(n)+d(m)} V(x)\left[1+u_{n, m}(x)\right]
$$

that is unique subject to the decay condition $u_{n, m}(x)=O\left(|x|^{2-d}\right)$ as $|x| \rightarrow \infty$. The quantities $\beta: \mathbb{N} \times \mathbb{N} \rightarrow(0, \infty)$ in (1.1) are then specified by the formula

$$
\beta(n, m)=\alpha(n, m) \int_{\mathbb{R}^{d}} V(x)\left(1+u_{n, m}(x)\right) d x .
$$

Our main result is conveniently expressed in terms of the empirical measures on the locations of particles of a given mass. For each $n \in \mathbb{N}$ and $t \in[0, \infty)$, we write $g_{n}(d x, t)$ for the random measure on $\mathbb{R}^{d}$ given by

$$
g_{n}(d x, t)=\varepsilon^{d-2} \sum_{i \in I_{q(t)}} \delta_{x_{i}(t)}(d x) \mathbb{1}\left(m_{i}(t)=n\right) .
$$

We write $g$ for the random measure on space-mass-time $\mathbb{R}^{d} \times \mathbb{N} \times[0, \infty)$ such that, for each $t \geq 0$, its time- $t$ marginal $g(\cdot, t)$ is given by

$$
g(\cdot, t)=\varepsilon^{d-2} \sum_{i \in I_{q(t)}} \delta_{\left(x_{i}(t), m_{i}(t)\right)} .
$$

We also require a mild hypothesis on the diffusion coefficients $d: \mathbb{N} \rightarrow(0, \infty)$. Namely, we suppose that there exists a function $\gamma: \mathbb{N}^{2} \rightarrow(0, \infty)$ such that $\alpha \leq \gamma$, with $\gamma$ satisfying

$$
n_{2} \gamma\left(n_{1}, n_{2}+n_{3}\right) \max \left\{1,\left[\frac{d\left(n_{2}+n_{3}\right)}{d\left(n_{2}\right)}\right]^{\frac{3 d-2}{2}},\left[\frac{d\left(n_{2}+n_{3}\right)}{d\left(n_{2}\right)}\right]^{2 d-1}\right\} \leq\left(n_{2}+n_{3}\right) \gamma\left(n_{1}, n_{2}\right) .
$$

The initial random configuration of $N$ particles is formed by scattering particles of numerous masses independently in $\mathbb{R}^{d}$ according to densities that are prescribed for each mass. These densities will be chosen as continuous functions $\left\{h_{n}: \mathbb{R}^{d} \rightarrow[0, \infty), n \in \mathbb{N}\right\}$, and should satisfy some fairly weak bounds. To be specific, we insist that

- $\bar{k} *(-\Delta)^{-1} k \in L_{l o c}^{\infty}\left(\mathbb{R}^{d}\right)$ where $k:=\sum_{n=1}^{\infty} n h_{n}$ and $\bar{k}(x)=k(-x)$

- For every $m, \sum_{n=1}^{\infty} d(n)^{d / 2-1 / 3} \gamma(m, n) \hat{h}_{n} \in L_{l o c}^{\infty}\left(\mathbb{R}^{d}\right)$ where $\hat{h}_{n}(x)=\int h_{n}(y)|x-y|^{-d+2 / 3} d y$.

- For every $\mathrm{m}, \sum_{n=1}^{\infty} d(n)^{d / 2-1 / 4} \gamma(m, n) \tilde{h}_{n} \in L_{l o c}^{\infty}\left(\mathbb{R}^{d}\right)$ where $\tilde{h}_{n}(x)=\int h_{n}(y)|x-y|^{-d+1 / 2} d y$.

We then set $Z=\sum_{n=1}^{\infty} \int_{\mathbb{R}^{d}} h_{n} \in(0, \infty)$ and choose $N$ points in $\mathbb{R}^{d} \times \mathbb{N}$ independently according to a law whose density at $(x, n)$ is equal to $h_{n}(x) / Z$. Selecting arbitrarily a set of $N$ symbols 
$\left\{i_{j}: j \in\{1, \ldots, N\}\right\}$ from $I$, we define the initial configuration $q_{0}$ by insisting that $q_{0}\left(i_{j}\right)$ is equal to the $j$-th of the randomly chosen members of $\mathbb{R}^{d} \times \mathbb{N}$.

Remark. It is not hard to show that our assumptions on the initial data $\left\{h_{n}\right\}$ are satisfied if $k$ is bounded, $k$ has a bounded support, $d(\cdot)$ is bounded, and $\gamma(m, n) \leq C(m) n$ for a function $C(\cdot)$. Indeed if $k$ is bounded and has a bounded support, then $\bar{k} *(-\Delta)^{-1} k \in L_{l o c}^{\infty}$ and $\hat{h}_{n}, \tilde{h}_{n} \in L_{l o c}^{\infty}$ for every $n$. It is worth mentioning that if $k$ belongs to the negative Sobolev Space $H^{-1}=W^{-1,2}$, then $\bar{k} *(-\Delta)^{-1} k \in L^{\infty}$.

The next statement is the main result of this paper.

Theorem 1.1. Let $d \geq 3$ and suppose that (1.9) holds. Let $\mathcal{P}_{N}$ denote the law on measures on $\mathbb{R}^{d} \times \mathbb{N} \times[0, \infty)$ given by the law of $g$ under $\mathbb{P}_{N}$; recall that $\epsilon$ is related to $N$ by means of the formula $N \epsilon^{d-2}=Z$, with the constant $Z \in(0, \infty)$ being given by the expression $Z=\sum_{n \in \mathbb{N}} \int_{\mathbb{R}^{d}} h_{n}$.

The sequence $\left\{\mathcal{P}_{N}\right\}$ is tight. Moreover, any limit point $\mathcal{P}$ of the sequence $\left\{\mathcal{P}_{N}\right\}$ is concentrated on the space of measures taking the form $\sum_{n=0}^{\infty} f_{n}(x, t) d x \times \delta_{n} \times d t$ where $\left\{f_{n}: n \in \mathbb{N}\right\}$ ranges over weak solutions of (1.1) that satisfy the initial condition $f_{n}(\cdot, 0)=h_{n}(\cdot)$; recall that the collection of constants $\beta: \mathbb{N}^{2} \rightarrow[0, \infty)$ is given by (1.8).

Theorem 1.1 describes the evolution of the density profiles of particles of various masses in the limit of large particle number by means of the Smoluchowski PDE. Note that, if the weak solution of this system of PDE is not known to be unique, we merely demonstrate convergence in a subsequential sense to the space of solutions. For example, admitting the possibility that the system (1.1) has two distinct weak solutions $\left\{f_{n}^{\prime}: n \in \mathbb{N}\right\}$ and $\left\{\hat{f}_{n}: n \in \mathbb{N}\right\}$ with initial condition $f_{n}(\cdot, 0)=h_{n}(\cdot)$, each of the following behaviours is consistent with Theorem 1.1;

- the empirical densities under the microscopic models $\mathbb{P}_{N}$ may converge weakly to the solution $\left\{f_{n}^{\prime}: n \in \mathbb{N}\right\}$ as $N \rightarrow \infty$ along the subsequence of even integers, and to $\left\{\hat{f}_{n}: n \in \mathbb{N}\right\}$ as $N \rightarrow \infty$ along the subsequence of odd integers;

- it may be that evolution of these densities is accurately approximated by flipping a fair coin, with the densities converging weakly to $\left\{f_{n}^{\prime}: n \in \mathbb{N}\right\}$ as $N \rightarrow \infty$ should the outcome be heads, and to $\left\{\hat{f}_{n}: n \in \mathbb{N}\right\}$ as $N \rightarrow \infty$ should the outcome be tails.

These peculiar scenarios are excluded if uniqueness of solutions to (1.1) is known. Some conditions for uniqueness are furnished by Proposition 2.6 of [11]; subsequently to the first appearance of the present work, [4 provided uniqueness under quite weak hypotheses. Indeed, as Remark 1.2 of [4] discusses, the next proposition is a consequence of Theorems 1.1, 1.2, 1.3 and 1.4 of [4].

Proposition 1. Let the dimension satisfy $d \geq 1$. For $a, b>0$ such that $a+b<1$, and for positive constants $c_{1}$ and $c_{2}$, assume that $\beta(n, m) \leq c_{1}\left(n^{a}+m^{a}\right)$ and $d(n) \geq c_{2} n^{-b}$ for all $n, m \in \mathbb{N}$. Also assume that $d: \mathbb{N} \rightarrow(0, \infty)$ is non-increasing. There exists $e>0$ such that $\sum_{n} n^{e}\left\|h_{n}\right\|_{L^{\infty}\left(\mathbb{R}^{d}\right)}<\infty$ and $\left\|\sum_{n} n^{e} h_{n}\right\|_{L^{1}\left(\mathbb{R}^{d}\right)}<\infty$ imply that (1.1) has a unique weak solution. (In fact, this solution conserves mass, in the sense that $I:[0, \infty) \rightarrow[0, \infty)$ given by $I(t)=\sum_{m \in \mathbb{N}} m \int_{\mathbb{R}^{d}} f_{m}(x, t) d x$ satisfies $I(t)=I(0)$ for all $t \in[0, \infty)$.) 
It is a simple corollary of Theorem 1.1 and Proposition 1 that convergence to (1.1) in fact holds in the following stronger sense.

Corollary 1. Let $d \geq 3$ and suppose that (1.9) and the assumptions of Proposition 1 are in force. Let $J: \mathbb{R}^{d} \times[0, \infty) \rightarrow \mathbb{R}$ be a bounded and continuous test function. Then, for each $n \in \mathbb{N}$ and $t \in(0, \infty)$,

$$
\limsup _{N \rightarrow \infty} \mathbb{E}_{N}\left|\int_{\mathbb{R}^{d}} J(x, t)\left(g_{n}(d x, t)-f_{n}(x, t) d x\right)\right|=0,
$$

where again $N \epsilon^{d-2}=Z$, with $Z=\sum_{n \in \mathbb{N}} \int_{\mathbb{R}^{d}} h_{n}$. In (1.10), $\left\{f_{n}: \mathbb{R}^{d} \times[0, \infty) \rightarrow[0, \infty), n \in \mathbb{N}\right\}$ denotes the unique weak solution to the system of partial differential equations (1.1), with $\beta: \mathbb{N}^{2} \rightarrow$ $[0, \infty)$ again given by (1.8).

\section{Remarks.}

- Included in the space of parameter values that satisfy (1.9) is the case where the diffusion rate $d$ is a decreasing function of the mass, and the coagulation propensities $\alpha$ satisfy $\alpha(n, m) \leq C n m$. In fact for a nonincreasing $d(\cdot)$, the condition (1.9) is equivalent to saying that $\alpha(n, m) \leq C(n) m$ for a function $C(n)$. Also, if the microscopic coagulation rate $\alpha$ is identically constant, then the condition (1.9) is equivalent to saying that the function $d(n) n^{1 /(2-3 d)}$ is nonincreasing.

- We believe that the derivation that we have undertaken is of particular interest, because the technique of proof that we adopt is robust under numerous changes in the details of the mechanism of interaction. An alternative model which we may treat is that where the mechanism of interaction is hard-core. In this variant, the reaction mechanism is deterministic, a pair of particles coagulating at the moment when their locations differ by $2 \epsilon$. The hard-core model with constant diffusion rates $d(\cdot) \equiv 1$ was treated in dimension $d=3$ in [6], with the macroscopic reaction propensity $\beta$ being identically given by the capacity $2 \operatorname{cap}\left(B_{0}(1)\right)$ of the unit-ball. Although we have not written the proof in this paper using a notation that includes the case of hard-core interaction, the method of proof yields the results of [6]. Indeed, we can prove the analogue of Theorem 1.1 for hard-core interaction in any dimension $d \geq 3$, provided that we may invoke the hypothesis

$$
n_{2} \max \left\{1,\left[\frac{d\left(n_{2}+n_{3}\right)}{d\left(n_{2}\right)}\right]^{\frac{3 d-2}{2}},\left[\frac{d\left(n_{2}+n_{3}\right)}{d\left(n_{2}\right)}\right]^{2 d-1}\right\} \leq n_{2}+n_{3} .
$$

The relation $\beta(n, m)=(d(n)+d(m)) \operatorname{cap}\left(B_{0}(1)\right)$ holds. The hard-core model is anyway approximated in terms of its macroscopic evolution by the stochastic one, with high choices for the constants $\alpha$, as we will see in Theorem [6.2 if the support of $V$ is the unit ball

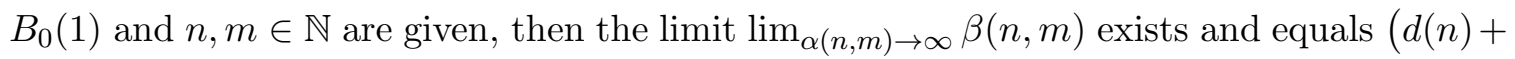
$d(m)) \operatorname{cap}\left(B_{0}(1)\right)$, the limit being taken with $d(n)$ and $d(m)$ fixed.

- Our technique of proof also yields a kinetic limit derivation for the model in which particles are assumed to have a range of interaction that is mass-dependent. To give an example of such variants, suppose that each particle of mass $m$ has a radius $r(m)$, where $r(m)=m^{\chi}$, with $\chi$ a fixed value satisfying $\chi \in(0,1 /(d-2))$. Assume, for the sake of brevity, that the diffusion rate $d$ is uniformly bounded above and below. We stipulate that particles of mass 
$m$ and $n$ are liable to react when their displacement reaches the order of $(r(m)+r(n)) \epsilon$. More precisely, we modify the definition (1.6) of the collision operator $\mathbb{A}_{C}$ by replacing the appearance of $V$ by $(r(n)+r(m))^{-2} V(\cdot /(r(n)+r(m)))$, (the factor that multiplies $V$ being introduced so that, roughly speaking, the altered collision mechanism respects the spatial-temporal scaling of Brownian motion). The statement of Theorem 1.1 is altered in that the macroscopic reaction propensities are given by (1.8) with $u_{n, m}$ solving (1.7) where $V$ is replaced by $(r(n)+r(m))^{-2} V(\cdot /(r(n)+r(m)))$. (The condition that $\chi$ be bounded above as stated is required in the proof of Lemma 3.3. Varying the details of this proof is the principal step required in extending our investigation to this altered model.) We mention also that investigating this model in the case where the radius $r$ is chosen to be a more rapidly increasing function of mass may be highly interesting, because it may be used to describe interacting systems of polymers having a fractal structure, in which a gelation effect occurs macroscopically, in the sense that the system of equations (1.1) lacks a well-defined solution after some finite time before which particles of high mass acculumate.

- In Section 2, we will discuss how the product of densities that appears in the interaction terms (1.2) and (1.3) of the macroscopic evolution is a consequence of the asymptotic independence for each positive time of particle locations in the large random models. As we will discuss, this independence fails to hold in a large microscopic vicinity of the location of a given particle: it is less probable that at any given positive time, a particle is present at a distance of the order of $\epsilon$ from a given particle than at a randomly chosen point in space, because the mechanism of coagulation has the effect of clearing space about any given particle. The endeavour of deriving the relation (1.8) between the macroscopic and microscopic reaction propensities has been a formidable one, since in so doing, we have determined precisely the nature of this negative microscopic correlation which forms a correction to the overall asymptotic independence of particle locations at positive times. The authors of [2] accomplished an analogous task for the quantum mechanical problem of a Bose-Einstein condensate with a quite singular choice of pair potential, in which the negative microscopic correlation corrects the Born approximation. An assumption that removed interaction between particles in small regions where several were present was employed.

We conclude this introduction by mentioning some previous work related to the problem.

As we just mentioned, the project of deriving the kinetic limit of the particle densities that we undertake was treated for the hard-core model, in the case where the diffusion rates are identically equal to a constant, in [6]. The approach of this paper was that by which Lanford [5] had derived for short times Boltzmann's equation as a similar limit of a system of balls that move at constant velocities and experienced elastic collisions. That is, the authors verified that the correlation functions of the particle locations satisfied the BBGKY hierarchy, a system of equations that may be derived formally for such systems by assuming sufficient independence in the location of particles. Similarly to Lanford's approach, the derivation in [6] was made on a short time interval. However, an iteration allowed it to be extended for all time. 
A related problem has been studied by Sznitman [9]. Brownian spheres diffuse and annihilate as soon as they touch. The partial differential equation by which the density of particles evolves was derived for the kinetic limit, in each dimension $d \geq 2$.

The stochastic coalescent is a random model in which particles - elements of an abstract set - are assigned a non-negative mass lying in $[0, \infty)$, and pairs of particles of mass $x$ and $y$ coagulate at an exponential rate $K(x, y)$. Norris [7] showed that for many choices of interaction rate $K$, in the limit of large initial particle number, the density of particles evolves in time according to a spatially homogeneous analogue of (1.1), where the densities depend on time alone and the Laplacian term is absent. The coagulation rate $\beta$ in this case is the same as in the random model: $\beta(x, y)=K(x, y)$. The derivation was valid provided that this system of partial differential equations had an unique solution, sufficient conditions for which were proved.

In [3], a derivation of a system similar to (1.1) is made from a microscopic model with a finite number of possible mass types, and a mechanism for interaction that is over a long-range compared to the microscopic scale.

In the next section, we give an overview of the proof of Theorem 1.1, describing the organisation of the paper as we do so.

Acknowledgment. The first author would like to thank James Norris for introducing him to the topic of diffusive coagulating systems and for valuable discussions.

\section{A SKETCH OF THE PROOF}

An essential element in the proof of Theorem 1.1 is to verify that the distribution of particles of two distinct masses is independent in the high $N$ limit. This is the so-called Stosszahlansatz of Boltzmann. Before sketching how we perform this task, we illustrate how assuming a form of the Stosszahlansatz tells us that the choice of scaling $N=\epsilon^{2-d} Z$ in the model identifies the regime of the bounded mean free path: that is, why the number of collisions experienced per unit time by a typical particle is of unit order, when $N$ is taken to be very large. In this regard, recall that the volume swept out by a ball of radius $\epsilon$ about the location of a particle in the first unit of time is that of the 'Wiener sausage' of parameter $\epsilon$, and is of the order of $\epsilon^{d-2}$. The number of collisions is of the order of the number of instances that, in this unit of time, other particles lie in this swept-out volume. The other particles are distributed independently at later times - at least, if we admit some form of the Stosszahlansatz - so that we expect the particle to encounter of the order of $N \epsilon^{d-2}$ other particles. The scaling of $N$ and $\epsilon$ ensures that this remains bounded above and away from zero as $N$ increases.

In the form in which we prove it, the Stosszahlansatz asserts that the total "propensity to coagulate' between particles of mass $M_{1}$ and $M_{2}$, weighted spatially by some time-dependent test functions $J, \bar{J}: \mathbb{R}^{d} \rightarrow[0, \infty) \times \mathbb{N} \times[0, \infty)$ in the first $T$ units of time,

$$
\frac{\epsilon^{d-2}}{2} \int_{0}^{T} \sum_{i, j \in I_{q}} V_{\epsilon}\left(x_{i}-x_{j}\right) \alpha\left(m_{i}, m_{j}\right) J\left(x_{i}, M_{1}, t\right) \bar{J}\left(x_{j}, M_{2}, t\right) \mathbb{1}\left\{m_{i}=M_{1}, m_{j}=M_{2}\right\} d t
$$


is close, when $N$ is high enough, to an expression involving a space-time integral of products of the candidate densities:

$$
\frac{\beta\left(M_{1}, M_{2}\right)}{2} \int_{0}^{T} \int_{\mathbb{R}^{d}} f^{\delta}\left(M_{1}, x, t\right) f^{\delta}\left(M_{2}, x, t\right) J\left(x, M_{1}, t\right) \bar{J}\left(x, M_{2}, t\right) d x d t .
$$

The precise statement of this claim appears in Section 3.5 as Proposition 2, In seeking to verify the claim, we introduce the random variables $Q(z)$, or, more fully, $Q\left(z, M_{1}, M_{2}, t\right)$, given by

$$
\begin{aligned}
\frac{\epsilon^{d-2}}{2} \sum_{i, j \in I_{q}} V_{\epsilon}\left(x_{i}(t)-x_{j}(t)+z\right) \alpha\left(m_{i}(t), m_{j}(t)\right) \\
J\left(x_{i}(t), M_{1}, t\right) \bar{J}\left(x_{j}(t), M_{2}, t\right) \mathbb{1}\left\{m_{i}(t)=M_{1}, m_{j}(t)=M_{2}\right\},
\end{aligned}
$$

where $z \in \mathbb{R}^{d}$. In the case of $z=0, Q(0)$ measures the average propensity per particle at time $t$ for particles of mass $M_{1}$ and $M_{2}$ to interact (with a spatial weighting due to the test functions). It is an average due to the appearance of the factor of $\epsilon^{d-2}$ in (2.3), whose reciprocal is of the order of the number of particles in the system initially. The total number of particles is expected to remain of this order at any given later time, because a typical particle collides with only finitely many others per unit time. This means that, ignoring a constant factor, we may think of $Q(0)$ as the average propensity to interact for any given value $t$ of the time parameter. We also consider expressions $\bar{Q}$, defined by the formula in (2.3), with the interaction kernel $V_{\epsilon}$ being replaced by another, $\hat{V}_{\epsilon}$, having similar properties. If $z$ is fixed at a small and constant value, while the parameter $\epsilon$ is taken to be much smaller, then any such $\bar{Q}(z)$ measures the extent of appearances at time $t$ of particles of mass $M_{2}$ lying in a microscopic ball of radius $\epsilon$ centred about a small macroscopic displacement $z$ from some mass $M_{1}$ particle. Instances of such pairs of particles are tentative candidates for collision after the passing of a short period of macroscopic time. As such, the assertion that, for fixed $t \in(0, \infty)$, the expression $\bar{Q}\left(z_{1}\right)-\bar{Q}\left(z_{2}\right)$ is small if $z_{1}$ and $z_{2}$ are small, captures the near independence of the locations of particles of distinct masses that is in essence the Stosszahlansatz . Indeed, we will demonstrate that, for a particular variant $\bar{Q}$ of $Q$,

$$
\lim _{z \downarrow 0} \mathbb{E}_{N}\left|\int_{0}^{T}(\bar{Q}(z)-Q(0)) d t\right|=0,
$$

the limit being taken in such a way that $\epsilon /|z| \rightarrow 0$ as $z \rightarrow 0$. Then, choosing $\eta^{\delta}$ to be a sequence that approximates the Dirac $\delta$ function, we find that $\int_{0}^{T} Q(0) d t$ is likely to be close to

$$
\int_{0}^{T} \int_{\mathbb{R}^{d}} \int_{\mathbb{R}^{d}} \bar{Q}\left(z_{1}-z_{2}\right) \eta^{\delta}\left(z_{1}\right) \eta^{\delta}\left(z_{2}\right) d z_{1} d z_{2} d t
$$

By substituting the expression in (2.3) (evaluated with the choice $z=z_{1}-z_{2}$, and with $\hat{V}_{\epsilon}$ replacing $V_{\epsilon}$ ) for $\bar{Q}\left(z_{1}-z_{2}\right)$, we find that the expression in (2.5) takes the form appearing in (2.2), that is, a time-averaged product of candidate densities. (The details, which appear in the proof of Proposition 2. are given in (3.74) ). We now turn to sketch how a statement of the form (2.4) is derived.

In a first effort to understand the difference in the behaviour of the time integral of the coagulation rate $Q(0)$ and its $z$-displaced counterpart $Q(z)$, we write down a $z$-dependent functional $S_{z}$ on configuration space for which the action of the diffusion operator $\mathbb{A}_{0}$ on $X_{z}$ produces, among 
others, the term $Q(z)$. For each pair $(n, m) \in \mathbb{N}^{2}$, we define $\phi_{n, m}^{\epsilon}: \mathbb{R}^{d} \rightarrow(0, \infty)$ by the stipulation that $\Delta \phi_{n, m}^{\epsilon}(x)=\alpha^{\prime}(n, m) \epsilon^{-d} V(z / \epsilon)$, where we write

$$
\alpha^{\prime}(n, m)=\alpha(n, m) /(d(n)+d(m)) .
$$

We then set

$$
S_{z}(q)=\epsilon^{2(d-2)} \sum_{i, j \in I_{q}} \phi_{M_{1}, M_{1}}^{\epsilon}\left(x_{i}-x_{j}+z\right) J\left(x_{i}, M_{1}, t\right) \bar{J}\left(x_{j}, M_{2}, t\right) \mathbb{1}\left\{m_{i}=M_{1}, m_{j}=M_{2}\right\} .
$$

When the operator $\mathbb{A}_{0}$ acts, we find that the term $Q(z)-Q(0)$ is obtained when the operator $\mathbb{A}_{0}$ acts on $S_{z}-S_{0}$ with each of the two derivatives of the Laplacian falling on $\phi^{\epsilon}$ rather than the test functions. Consider the identity

$$
\begin{gathered}
\left(S_{z}-S_{0}\right)(T)=\left(S_{z}-S_{0}\right)(0)+\int_{0}^{T}\left(\frac{\partial}{\partial t}+\mathbb{A}_{0}\right)\left(S_{z}-S_{0}\right)(t) d t \\
+\int_{0}^{T} \mathbb{A}_{C}\left(S_{z}-S_{0}\right)(t) d t+M_{T},
\end{gathered}
$$

and note that the process $\left\{M_{T}: T \geq 0\right\}$ is a martingale. We know that two terms lie inside this identity that remain of unit order as we take a limit in low $z$ (with $\epsilon$ tending to 0 much more quickly). These are $\int_{0}^{T} Q(z) d t$ and $-\int_{0}^{T} Q(0) d t$. Were the only terms to remain of unit order to be those two, we would deduce a conclusion of the form we seek, namely (2.4) where $\bar{Q}$ is simply $Q$. However, (2.4) cannot be valid for this choice of $\bar{Q}$. Indeed, imagine that $\alpha\left(M_{1}, M_{2}\right)$ is a very large constant. Particles of order $\epsilon$ apart would typically coagulate in a very small fraction of the order $\epsilon^{2}$ of time in which they are likely to remain at a distance of order $\epsilon$. At the moment that the pair of particles vanishes, it ceases to contribute to the expression $Q(0)$, meaning that, at any particular time $t \in[0, \infty), Q(0)$ need not be large, even if the constant $\alpha\left(M_{1}, M_{2}\right)$ is. However, for fixed $z$, pairs of particles, one of which lies in an $\epsilon$-ball about the $z$-displacement of the other, may remain in this state for a significant constant multiple of $\epsilon^{2}$ of upcoming time. This means that $Q(z)$ is likely to be huge, if $\alpha\left(M_{1}, M_{2}\right)$ is.

In fact, there is another term that remains of unit order in (2.8) in the low $z$ limit. This term arises from the action of the collision operator $\mathbb{A}_{C}$ on $S_{0}$. Two or three particles may index the summands of such terms. Two such particles are those that may coagulate, appearing in the sum associated to the collision operator. There may or may not be another occurring as a third particle in the term $S_{0}$. It is the case of the term corresponding to only two particles that remains of unit order. It takes the form

$$
\epsilon^{2(d-2)} \alpha\left(M_{1}, M_{2}\right) \sum_{k, l \in I_{q}} V_{\epsilon}\left(x_{k}-x_{l}\right) \phi_{M_{1}, M_{2}}^{\epsilon}\left(x_{k}-x_{l}\right) J\left(x_{k}, M_{1}\right) \bar{J}\left(x_{l}, M_{2}\right) .
$$

This term witnesses the abrupt curtailment of the propensity to coagulate of a pair of particles at that moment when they do coagulate. Given the argument just presented against the possibility that $\bar{Q}(0)$ is equal to $Q(0)$ in (2.4), it is not surprising that this extra term should arise in this way.

There are numerous other terms in (2.8). It turns out that each of them vanishes in the limit of low z. Accepting for the time being that this is so, we have however not quite arrived at a 
conclusion of the form we seek. What we have found is that $\int_{0}^{T} Q(z) d t$ is close, when $z$ is small, to the expression

$$
\begin{aligned}
\epsilon^{2(d-2)} \alpha\left(M_{1},\right. & \left.M_{2}\right) \int_{0}^{T} d t \sum_{i, j \in I_{q}} V_{\epsilon}\left(x_{k}-x_{l}\right) \\
& \times\left[1-\phi_{M_{1}, M_{2}}^{\epsilon}\left(x_{i}-x_{j}\right)\right] J\left(x_{i}, M_{1}\right) \bar{J}\left(x_{j}, M_{2}\right) \mathbb{1}\left\{m_{i}=M_{1}, m_{j}=M_{2}\right\} .
\end{aligned}
$$

The ' 1 ' that appears in the square bracket corresponds to $Q(0)$, and the other term to the unit order term (2.9). It was our aim to show that $\int_{0}^{T} Q(0) d t$ was close to an expression of the form $\int_{0}^{T} \bar{Q}(z) d t$, but our first try gave us such a statement with the roles of $Q$ and $\bar{Q}$ reversed. We are led therefore to consider an altered functional $X_{z}$ of the configuration space:

$$
X_{z}(q)=\epsilon^{2(d-2)} \sum_{i, j \in I_{q}} u_{M_{1}, M_{2}}^{\epsilon}\left(x_{i}-x_{j}+z\right) J\left(x_{i}, M_{1}, t\right) \bar{J}\left(x_{j}, M_{2}, t\right) \mathbb{1}\left\{m_{i}=M_{1}, m_{j}=M_{2}\right\} .
$$

We have replaced the appearance of $\phi_{m_{i}, m_{j}}^{\epsilon}\left(x_{i}-x_{j}+z\right)$ by that of $u_{m_{i}, m_{j}}^{\epsilon}\left(x_{i}-x_{j}+z\right)$ to move from (2.7) to (2.11). Note that the analogue of the expression (2.10) in this case is given by

$$
\begin{gathered}
\epsilon^{2(d-2)} \sum_{i, j \in I_{q}}\left[\left(d\left(M_{1}\right)+d\left(M_{2}\right)\right) \Delta u_{M_{1}, M_{2}}^{\epsilon}\left(x_{i}-x_{j}\right)-\alpha\left(M_{1}, M_{2}\right) V_{\epsilon}\left(x_{i}-x_{j}\right) u^{\epsilon}\left(x_{i}-x_{j}\right)\right] \\
J\left(x_{i}, M_{1}\right) \bar{J}\left(x_{j}, M_{2}\right) \mathbb{1}\left\{m_{i}=M_{1}, m_{j}=M_{2}\right\} .
\end{gathered}
$$

We aim to define the function $u_{M_{1}, M_{2}}^{\epsilon}$ in such a way that this term is equal to $Q(0)$. This means that $u_{M_{1}, M_{2}}^{\epsilon}$ should satisfy

$$
\left(d\left(M_{1}\right)+d\left(M_{2}\right)\right) \Delta u_{M_{1}, M_{2}}^{\epsilon}(x)=\alpha\left(M_{1}, M_{2}\right) V_{\epsilon}(x) u^{\epsilon}(x)+\alpha\left(M_{1}, M_{2}\right) V^{\epsilon}(x)
$$

for each $x \in \mathbb{R}^{d}$. Writing $u_{M_{1}, M_{2}}^{\epsilon}(x)=\epsilon^{2-d} u_{M_{1}, M_{2}}(x / \epsilon)$ and recalling the definition (2.6) of the constants $\alpha^{\prime}\left(M_{1}, M_{2}\right)$, the equation (2.13) for any, or all, $\epsilon>0$, is equivalent to the requirement that $u_{M_{1}, M_{2}}: \mathbb{R}^{d} \rightarrow \mathbb{R}$ satisfies

$$
\Delta u_{M_{1}, M_{2}}(x)=\alpha^{\prime}\left(M_{1}, M_{2}\right) V(x)\left[1+u_{M_{1}, M_{2}}(x)\right] .
$$

In Section 6, we prove that the equation (2.14) has an unique solution in $C^{2}\left(\mathbb{R}^{d}\right)$. This piece of theory allows us to define the functional $X_{z}$ so that it has the desired effect: the term $\int_{0}^{T} Q(0) d t$ is close to $\int_{0}^{T} \bar{Q}(z) d t$ for small $z$. The form of $\bar{Q}(z)$ is given by

$\epsilon^{2(d-2)} \sum_{i, j \in I_{q}}\left(d\left(M_{1}\right)+d\left(M_{2}\right)\right) \Delta u_{M_{1}, M_{2}}^{\epsilon}\left(x_{i}-x_{j}+z\right) J\left(x_{i}, M_{1}\right) \bar{J}\left(x_{j}, M_{2}\right) \mathbb{1}\left\{m_{i}(t)=M_{1}, m_{j}(t)=M_{2}\right\}$.

This expression explains the form of the modification as we pass from the microscopic propensity to coagulate, given by $\alpha\left(M_{1}, M_{2}\right)$, to its macroscopic analogue $\beta\left(M_{1}, M_{2}\right)$. The recipe (1.8) for computing $\beta$ is nothing other than

$$
\beta\left(M_{1}, M_{2}\right)=\left(d\left(M_{1}\right)+d\left(M_{2}\right)\right) \int_{\mathbb{R}^{d}} \Delta u_{M_{1}, M_{2}}(x) d x
$$

an equation that arises because for small $z$, the significant behaviour in (2.15) is given by that of $u(x-y)$ on its diagonal $x=y$. 
Our task then becomes that of examining the analogue of the identity (2.8) in the case where $S_{z}$ is replaced by $X_{z}$. Estimates are required to prove that the remaining terms, including that of the martingale, vanish in the low $z$-limit. It would suffice in this regard to demonstrate the following form of independence in the behaviour of the particles: that there exists a large constant $C$ such that, for any $n \in \mathbb{N}$ and $t \in(0, \infty)$, if $n$ particles are picked independently at random from the initial configuration, then the probability that all of them lie in a given small ball of radius $r$ at time $t$ is bounded above by $C r^{n}$. Although this statement may seem no easier to prove than the Stosszahlansatz, there is a special case for which it may be obtained by a coupling argument. This case is the one treated by [6], when all of the particles are supposed to have the same diffusion rate (although the argument presented here does not require the mechanism of coagulation to be of hard-core type). With this assumption, consider an altered model in which there are particles of two types, those living and those perished. Each particle is living at the beginning. Collisions may occur only between living particles. When a collision occurs, one particle remains alive and the other perishes, with each particle having an equal chance of surviving, independently of other randomness. Particles of either type diffuse according to independent Brownian motions at one given rate. The ensemble of all particles is a system of independently evolving Brownian motions, and is at any given time a superset of the living particles, which evolve according to our collision dynamics. The estimate mentioned follows straightaway from this fact.

In general, however, no such approach is available. After labelling the various terms arising from (2.8) at the beginning of Section 3, we instead establish various weakened statements in Subsection 3.1. of which that in Lemma 3.3 is an example whose proof is now discussed. It asserts that

$$
\mathbb{E}_{N} \int_{0}^{T} d t \sum_{k, l, i \in I_{q}} K_{n_{1}, n_{2}, n_{3}}\left(x_{k}, x_{l}, x_{i}\right) \mathbb{1}\left\{m_{k}=n_{1}, m_{i}=n_{3}\right\} \leq C \epsilon^{6-2 d},
$$

where $K$ is an instance of a smooth function mapping $\mathbb{R}^{3 d}$ to $[0, \infty)$, and where $C$ is a constant that depends on $n_{1}, n_{3}$ and $K$. The approach to proving such statements as (2.16) has much in common with the global one by which (2.4) is being obtained. We define a function $A_{n_{1}, n_{2}, n_{3}}^{\epsilon}: \mathbb{R}^{3 d} \rightarrow[0, \infty)$ on which the action of the diffusion operator $\mathbb{A}_{0}$ yields the generic summand in (2.16). That is, we insist that

$$
\left(d\left(n_{1}\right) \Delta_{1}+d\left(n_{2}\right) \Delta_{2}+d\left(n_{3}\right) \Delta_{3}\right) A_{n_{1}, n_{2}, n_{3}}^{\epsilon}\left(x_{1}, x_{2}, x_{3}\right)=-K_{n_{1}, n_{2}, n_{3}}^{\epsilon}\left(x_{1}, x_{2}, x_{3}\right) .
$$

We consider the action of the whole Markov generator $\mathbb{A}_{0}+\mathbb{A}_{C}$ on the sum $A_{n_{1}, n_{2}, n_{3}}\left(x_{i}, x_{j}, x_{k}\right)$ over those triples of indices $(i, j, k)$ for which $m_{i}=n_{1}$ and $m_{k}=n_{3}$. We work inductively in the lexicographical ordering in $\mathbb{N}^{2}$ for the pair $\left(n_{1}, n_{3}\right)$. The action of $\mathbb{A}_{0}$ on this sum naturally provides the expression that we seek to bound in (2.16). The action of the collision operator $\mathbb{A}_{C}$ produces three types of terms, that might be called gains, losses or internal terms. The gains and losses arise from collisions that produce or destroy particles of mass $n_{1}$ or $n_{3}$ that contribute to the expression in (2.16) in its first or third arguments. The gain term appears in an upper bound for this expression, and so must be bounded from above. Such a bound is provided by the sum of the loss terms from particles of lower mass, terms which are themselves bounded by the inductive hypothesis. The internal term arises from coagulations that remove two particles that contribute terms in the left-hand-side of (2.16) by means of the unrestricted second argument of $K$, and produce a new particle that itself contributes such a term. We argue that the internal term is 
non-positive, because the term corresponding to the new particle is less than the sum of the terms corresponding to the two that are disappearing. It is at this point that the hypothesis (1.9) on the model's parameters is used.

Some additional lemmas presenting straightforward bounds on the function $u^{\epsilon}$ are given in Subsection 3.2. For the analogue of (2.8) for the process $X_{z}-X_{0}$, estimates showing that numerous terms are negligible in the low $\epsilon$ limit are given in Subsection 3.3. The martingale term is similarly shown to be small in Subsection 3.4. In this way, the statement (2.4) is derived. The derivation of the Stosszahlansatz, as described after (2.4), is given in Subsection 3.5.

We aim to show that the macroscopic mass densities evolve according to the system of partial differential equations (1.1) as follows. In Section 5, we define the functional

$$
Y_{q}=\epsilon^{d-2} \sum_{i \in I_{q}} J\left(x_{i}, m_{i}, t\right)
$$

where $J: \mathbb{R}^{d} \times \mathbb{N} \times[0, \infty) \rightarrow \mathbb{R}$ denotes a test function. Consider the identity

$$
Y_{q}(T)=Y_{q}(0)+\int_{0}^{T} \mathbb{A}_{C}\left(Y_{q}\right)(t) d t+\int_{0}^{T}\left(\frac{\partial}{\partial t}+\mathbb{A}_{0}\right)\left(Y_{q}\right)(t) d t+M_{T}
$$

with the process $\left\{M_{T}: T \geq 0\right\}$ being a martingale. After demonstrating that the martingale term is negligible by arguments similar to those employed for the martingale arising from the previous functional $X_{z}$, we consider the identity obtained from (2.17) in the limit of low $\epsilon$. We expect to derive the system (1.1) in weak form, with the function $J$ playing the role of the test function. As discussed after (2.4), the Stosszahlansatz enables us to replace the term involving the collision operator in (1.1) by a time-averaged product of microscopic candidates for the density, that take the form of the empirical measure for particles of a given mass mollified on some scale $\delta$. To pass to the limit in low $\delta$ after $\epsilon$ has vanished and in this way obtain the weak form of (1.1), we make use of the fact that these empirical measures concentrate in the limit of low $\epsilon$ on measures having densities that are uniformly bounded with respect to Lebesgue measure. This fact is proved in Section 4 ,

We conclude this overview by elaborating the analogy between this work and that of [2] that was mentioned in a remark after Theorem 1.1. The authors of [2] consider a Bose-Einstein condensate with a potential between each pair of particles of the form $N^{2} V\left(N\left(x_{i}-x_{j}\right)\right)$, these particles having locations $x_{i}$ and $x_{j}$ in 3 -space. It is proved that the densities matrices in the problem satisfy an infinite BBGKY hierarchy of equations known as the Gross-Pitaevskii hierarchy. In our context, the assumption that the Stosszahlansatz holds in the naive form that $Q(z)-Q(0)$ is small for small $z$ would lead to a derivation of the Smoluchowski PDE (1.1) with macroscopic reaction rates $\beta(n, m)$ being incorrectly computed as being equal to the microscopic ones $\alpha(n, m)$. In [2], an analogously crude assumption that the two-particle density matrices may be expressed as a product of one-particle matrices yields the GP-hierarchy with an incorrect coupling constant equal to that which arises by making use of the Born approximation. In each of these contexts, it is necessary to understand the nature of the microscopic adjustment to particle independence in the vicinity of each particle to determine correctly the revelant macroscopic constants. The form of the 
adjustment is very similar between the two problems. It would be of much interest to determine why this is so.

\section{Establishing the Stosszahlansatz}

Recall that, for any given pair $(n, m) \in \mathbb{N}^{2}, u^{\epsilon}=u_{n, m}^{\epsilon}: \mathbb{R}^{d} \rightarrow[0, \infty)$ is the unique function whose existence is ensured by Theorem 6.1 that lies in $C^{2}\left(\mathbb{R}^{d}\right)$ satisfying $\lim _{x \rightarrow \infty} u_{n, m}^{\epsilon}(x)=0$ and

$$
\Delta u_{n, m}^{\epsilon}(x)=\alpha^{\prime}(n, m)\left[V_{\epsilon}(x) u_{n, m}^{\epsilon}(x)+V^{\epsilon}(x)\right],
$$

where the constants $\alpha^{\prime}$ were defined in (2.6) . We are using the notations

$$
V^{\epsilon}(x)=\epsilon^{-d} V\left(\frac{x}{\epsilon}\right), \quad V_{\epsilon}(x)=\epsilon^{-2} V\left(\frac{x}{\epsilon}\right) .
$$

Note that $u_{n, m}^{\epsilon}: \mathbb{R}^{d} \rightarrow[0, \infty)$ is given by

$$
u_{n, m}^{\epsilon}(x)=-c_{0}(d) \int_{\mathbb{R}^{d}} \frac{V^{\epsilon}(y)}{|x-y|^{d-2}} \alpha^{\prime}(n, m)\left[u_{n, m}\left(\frac{y}{\epsilon}\right)+1\right] d y,
$$

where $c_{0}=c_{0}(d)=(d-2)^{-1} \omega_{d}^{-1}$ with $\omega_{d}$ denoting the surface area of the unit sphere $S^{d-1}$. We are denoting by $u_{n, m}$ the function obtained by the choice $\epsilon=1$. We present the conditions on the two test functions $J, \bar{J}: \mathbb{R}^{d} \times \mathbb{N} \times[0, \infty) \rightarrow \mathbb{R}$ that appear in (2.7). It suffices to work with functions that take non-zero values for only one value in the second argument, such functions measuring the presence of particles of a given mass. By a temporary abuse of notation, we write

$$
J\left(x, M_{1}, t\right)=J(x, t) \mathbb{1}\left\{m=M_{1}\right\} \quad \text { and } \quad \bar{J}\left(x, M_{1}, t\right)=\bar{J}(x, t) \mathbb{1}\left\{m=M_{2}\right\},
$$

where on the right-hand-side, $J$ and $\bar{J}$ denote smooth maps from $\mathbb{R}^{d} \times[0, \infty)$ to $\mathbb{R}$ of compact support. We will suppress the appearance of the $t$-variable when writing the arguments of $J$ and $\bar{J}$.

Numerous terms arise when the operators $\mathbb{A}_{0}$ and $\mathbb{A}_{C}$ act on the expression $X_{z}-X_{0}$ (recall that the functions of configurations $X_{z}$, indexed by $z \in \mathbb{R}^{d}$, were defined in (2.8) ). We now label these terms. Unless stated otherwise, we will adopt a notation whereby all the index labels appearing in sums should be taken to be distinct. This includes the case of multiple sums. For example, $\sum_{k, l \in I_{q}} \sum_{i \in I_{q}} f\left(x_{k}, x_{l}, x_{i}\right)$ denotes the sum of the evaluation of the function $f$ over all arguments that are triples $\left(x_{k}, x_{l}, x_{i}\right)$ where $k, l$ and $i$ are distinct indices in $I$. Note also that, unless otherwise stated, whenever the symbol $u^{\epsilon}$ appears in a summand, we mean $u_{M_{1}, M_{2}}^{\epsilon}$.

Firstly, we label those terms arising from the action of the diffusion operator. To do so, note that, for a time-dependent functional $F$ of the configuration space, this action is given by

$$
\left(\frac{\partial}{\partial t}+\mathbb{A}_{0}\right) F=\frac{\partial}{\partial t} F+\sum_{i \in I_{q}} d\left(m_{i}\right) \Delta_{x_{i}} F .
$$

Thus, we label as follows:

$$
\left(\frac{\partial}{\partial t}+\mathbb{A}_{0}\right)\left(X_{z}-X_{0}\right)(q(t))=H_{11}+H_{12}+H_{13}+H_{14}+H_{2}+H_{3}+H_{4}
$$


with

$$
\begin{aligned}
H_{11}= & \epsilon^{2(d-2)} \sum_{i, j \in I_{q}} \alpha\left(m_{i}, m_{j}\right)\left[V^{\epsilon}\left(x_{i}-x_{j}+z\right)-V^{\epsilon}\left(x_{i}-x_{j}\right)\right] J\left(x_{i}, m_{i}, t\right) \bar{J}\left(x_{j}, m_{j}, t\right) \\
H_{12}= & -\epsilon^{2(d-2)} \sum_{i, j \in I_{q}} \alpha\left(m_{i}, m_{j}\right) V_{\epsilon}\left(x_{i}-x_{j}\right) u^{\epsilon}\left(x_{i}-x_{j}\right) J\left(x_{i}, m_{i}, t\right) \bar{J}\left(x_{j}, m_{j}, t\right) \\
H_{13}= & \epsilon^{2(d-2)} \sum_{i, j \in I_{q}} \alpha\left(m_{i}, m_{j}\right) V_{\epsilon}\left(x_{i}-x_{j}+z\right) u^{\epsilon}\left(x_{i}-x_{j}+z\right) J\left(x_{i}, m_{i}, t\right) \bar{J}\left(x_{j}, m_{j}, t\right), \\
H_{14}= & \epsilon^{2(d-2)} \sum_{i, j \in I_{q}}\left[u^{\epsilon}\left(x_{i}-x_{j}+z\right)-u^{\epsilon}\left(x_{i}-x_{j}\right)\right] \\
& {\left[J_{t}\left(x_{i}, m_{i}, t\right) \bar{J}\left(x_{j}, m_{j}, t\right)+J\left(x_{i}, m_{i}, t\right) \bar{J}_{t}\left(x_{j}, m_{j}, t\right)\right], }
\end{aligned}
$$

along with

$$
\begin{aligned}
& H_{2}=2 \epsilon^{2(d-2)} \sum_{i, j \in I_{q}} d\left(m_{i}\right) \bar{J}\left(x_{j}, m_{j}, t\right)\left[u_{x}^{\epsilon}\left(x_{i}-x_{j}+z\right)-u_{x}^{\epsilon}\left(x_{i}-x_{j}\right)\right] \cdot J_{x}\left(x_{i}, m_{i}, t\right) \\
& H_{3}=2 \epsilon^{2(d-2)} \sum_{i, j \in I_{q}} d\left(m_{j}\right) J\left(x_{i}, m_{i}, t\right)\left[u_{x}^{\epsilon}\left(x_{i}-x_{j}+z\right)-u_{x}^{\epsilon}\left(x_{i}-x_{j}\right)\right] \cdot \bar{J}_{x}\left(x_{j}, m_{j}, t\right),
\end{aligned}
$$

and

$$
\begin{aligned}
H_{4}=\epsilon^{2(d-2)} \sum_{i, j \in I_{q}} & {\left[u^{\epsilon}\left(x_{i}-x_{j}+z\right)-u^{\epsilon}\left(x_{i}-x_{j}\right)\right] } \\
& {\left[d\left(m_{i}\right) \Delta_{x} J\left(x_{i}, m_{i}, t\right) \bar{J}\left(x_{j}, m_{j}, t\right)+d\left(m_{j}\right) J\left(x_{i}, m_{i}, t\right) \Delta_{x} \bar{J}\left(x_{j}, m_{j}, t\right)\right], }
\end{aligned}
$$

where $f_{x}$ denotes the gradient of $f$, and - the scalar product. As for those terms arising from the action of the collision operator,

$$
\mathbb{A}_{C}\left(X_{z}-X_{0}\right)(q)=G_{z}(1)+G_{z}(2)-G_{0}(1)-G_{0}(2),
$$


where $G_{z}(1)$ is set equal to

$$
\begin{gathered}
\frac{1}{2} \sum_{k, l \in I_{q}} \alpha\left(m_{k}, m_{l}\right) V_{\epsilon}\left(x_{k}-x_{l}\right) \epsilon^{2(d-2)} \sum_{i \in I_{q}} \\
\left\{\frac { m _ { k } } { m _ { k } + m _ { l } } \left[u^{\epsilon}\left(x_{k}-x_{i}+z\right) J\left(x_{k}, m_{k}+m_{l}, t\right) \bar{J}\left(x_{i}, m_{i}, t\right)\right.\right. \\
\left.+u^{\epsilon}\left(x_{i}-x_{k}+z\right) J\left(x_{i}, m_{i}, t\right) \bar{J}\left(x_{k}, m_{k}+m_{l}, t\right)\right] \\
+\frac{m_{l}}{m_{k}+m_{l}}\left[u^{\epsilon}\left(x_{l}-x_{i}+z\right) J\left(x_{l}, m_{k}+m_{l}, t\right) \bar{J}\left(x_{i}, m_{i}, t\right)\right. \\
\left.+u^{\epsilon}\left(x_{i}-x_{l}+z\right) J\left(x_{i}, m_{i}, t\right) \bar{J}\left(x_{l}, m_{k}+m_{l}, t\right)\right] \\
-\left[u^{\epsilon}\left(x_{k}-x_{i}+z\right) J\left(x_{k}, m_{k}, t\right) \bar{J}\left(x_{i}, m_{i}, t\right)\right. \\
\left.+u^{\epsilon}\left(x_{i}-x_{k}+z\right) J\left(x_{i}, m_{i}, t\right) \bar{J}\left(x_{k}, m_{k}, t\right)\right] \\
-\left[u^{\epsilon}\left(x_{l}-x_{i}+z\right) J\left(x_{l}, m_{l}, t\right) \bar{J}\left(x_{i}, m_{i}, t\right)\right. \\
\left.\left.+u^{\epsilon}\left(x_{i}-x_{l}+z\right) J\left(x_{i}, m_{i}, t\right) \bar{J}\left(x_{l}, m_{l}, t\right)\right]\right\}
\end{gathered}
$$

and where

$$
G_{z}(2)=-\epsilon^{2(d-2)} \sum_{k, l \in I_{q}} \alpha\left(m_{k}, m_{l}\right) V_{\epsilon}\left(x_{k}-x_{l}\right) u^{\epsilon}\left(x_{k}-x_{l}+z\right) J\left(x_{k}, m_{k}, t\right) \bar{J}\left(x_{l}, m_{l}, t\right) .
$$

The expression in (2.9) is an analogue of $G_{0}(2)$. The terms in $G_{z}(1)$ arise from the changes in the functional $X_{z}$ when a collision occurs due to the influence of the appearance and disppearance of particles on other particles that are not directly involved. Those in $G_{z}(2)$ are due to the absence after collision of the summand in $X_{z}$ indexed by the colliding particles.

Note that

$$
H_{12}+G_{0}(2)=0
$$

The process $\left\{\left(X_{z}-X_{0}\right)(t): t \geq 0\right\}$ satisfies

$$
\begin{gathered}
\left(X_{z}-X_{0}\right)(T)=\left(X_{z}-X_{0}\right)(0)+\int_{0}^{T}\left(\frac{\partial}{\partial t}+\mathbb{A}_{0}\right)\left(X_{z}-X_{0}\right)(t) d t \\
+\int_{0}^{T} \mathbb{A}_{C}\left(X_{z}-X_{0}\right)(t) d t+M(T)
\end{gathered}
$$


with $\{M(t): t \geq 0\}$ being a martingale. By using the labels for the various terms that we just introduced, we find from (3.8) by use of (3.7) that

$$
\begin{aligned}
& \left|\int_{0}^{T} H_{11}(t) d t+\int_{0}^{T} H_{13}(t) d t\right| \\
\leq & \left|X_{z}-X_{0}\right|(q(T))+\left|X_{z}-X_{0}\right|(q(0)) \\
& +\int_{0}^{T}\left|H_{14}\right|(t) d t+\int_{0}^{T}\left|H_{2}\right|(t) d t+\int_{0}^{T}\left|H_{3}\right|(t) d t+\int_{0}^{T}\left|H_{4}\right|(t) d t \\
& +\int_{0}^{T}\left|G_{z}(1)-G_{0}(1)\right|(t) d t+\int_{0}^{T}\left|G_{z}(2)\right|(t) d t+|M(T)| .
\end{aligned}
$$

Since $J$ is of compact support, we have that $X_{z}(q(T))=0$ for $T$ sufficiently large.

We denote by $\mathbb{P}_{N}$ the measure on functions from $t \in[0, \infty)$ to the configurations determined by the process at time $t$. Its expectation will be denoted $\mathbb{E}_{N}$. We aim to prove the following estimates: for each $T>0$,

$$
\begin{aligned}
& \int_{0}^{T} \mathbb{E}_{N}\left|H_{14}\right|(t) d t \leq C|z|^{\frac{2}{d+1}}, \\
& \int_{0}^{T} \mathbb{E}_{N}\left|H_{2}\right|(t) d t \leq C|z|^{\frac{1}{d+1}}, \\
& \int_{0}^{T} \mathbb{E}_{N}\left|H_{3}\right|(t) d t \leq C|z|^{\frac{1}{d+1}}, \\
& \int_{0}^{T} \mathbb{E}_{N}\left|H_{4}\right|(t) d t \leq C|z|^{\frac{2}{d+1}} \\
& \int_{0}^{T} \mathbb{E}_{N}\left|G_{z}(1)-G_{0}(1)\right|(t) d t \leq C|z|^{\frac{2}{d+1}}, \\
& \int_{0}^{T} \mathbb{E}_{N}\left|G_{z}(2)\right|(t) d t \leq C\left(\frac{\epsilon}{|z|}\right)^{d-2}, \\
& \mathbb{E}_{N}\left|X_{z}-X_{0}(0)\right| \leq C|z| .
\end{aligned}
$$

Later we apply the limit $|z| \rightarrow 0$ in such a way that $\frac{\epsilon}{|z|} \rightarrow 0$. We will also show that, for each $T \in(0, \infty)$,

$$
\mathbb{E}_{N}\left[M(T)^{2}\right] \leq C \varepsilon^{d-2}
$$

3.1. Lemmas bounding collision propensity. We prove three lemmas:

Lemma 3.1. For any $T \in[0, \infty)$,

$$
\epsilon^{d-2} \mathbb{E}_{N} \int_{0}^{T} d t \sum_{i, j \in I_{q}} \alpha\left(m_{i}, m_{j}\right) V_{\epsilon}\left(x_{i}-x_{j}\right) \leq 2 Z .
$$


Lemma 3.2. Let $J: \mathbb{R}^{d} \rightarrow[0, \infty)$ be continuous, and let $H: \mathbb{R}^{d} \rightarrow[0, \infty)$ be a twice differentiable function such that $-\Delta H=J$. Then we have the following inequalities,

$$
\begin{gathered}
\mathbb{E}_{N} \int_{0}^{T} \epsilon^{2(d-2)} \sum_{i, j \in I_{q}} J\left(x_{i}-x_{j}\right) m_{i} m_{j}\left(d\left(m_{i}\right)+d\left(m_{j}\right)\right) d t \leq \epsilon^{2(d-2)} \mathbb{E}_{N} \sum_{i, j \in I_{q(0)}} H\left(x_{i}-x_{j}\right) m_{i} m_{j}, \\
\mathbb{E}_{N} \int_{0}^{T} \epsilon^{2(d-2)} \sum_{i, j \in I_{q}} V_{\epsilon}\left(x_{i}-x_{j}\right) \alpha\left(m_{i}, m_{j}\right) m_{i} m_{j} H\left(x_{i}-x_{j}\right) d t \leq \epsilon^{2(d-2)} \mathbb{E}_{N} \sum_{i, j \in I_{q(0)}} H\left(x_{i}-x_{j}\right) m_{i} m_{j} .
\end{gathered}
$$

Remark The fact that the dimension $d$ is at least three implies that $H \geq 0$.

Lemma 3.3. Assume that the function $\gamma: \mathbb{N}^{2} \rightarrow(0, \infty)$ satisfies

$$
n_{2} \gamma\left(n_{1}, n_{2}+n_{3}\right) \max \left\{1,\left[\frac{d\left(n_{2}+n_{3}\right)}{d\left(n_{2}\right)}\right]^{\frac{3 d-2}{2}}\right\} \leq\left(n_{2}+n_{3}\right) \gamma\left(n_{1}, n_{2}\right) .
$$

There exists a collection of constants $C: \mathbb{N}^{2} \rightarrow(0, \infty)$, such that, for any smooth function $J$ : $\mathbb{R}^{2 d} \rightarrow[0, \infty)$, and any given $n_{1}, n_{3} \in \mathbb{N}$,

$$
\begin{aligned}
& \mathbb{E}_{N} \int_{0}^{T} d t \sum_{k, j, i \in I_{q(t)}} \gamma\left(m_{i}, m_{j}\right) V_{\epsilon}\left(x_{i}-x_{j}\right) J\left(x_{i}, x_{k}\right) \mathbb{1}\left\{m_{i}=n_{1}, m_{k}=n_{3}\right\} \\
\leq & \epsilon^{3(2-d)} C_{n_{1}, n_{3}} \sum_{n_{2} \in \mathbb{N}} \int A_{n_{1}, n_{2}, n_{3}}^{\epsilon}\left(x_{1}, x_{2}, x_{3}\right) h_{n_{1}}\left(x_{1}\right) h_{n_{2}}\left(x_{2}\right) h_{n_{3}}\left(x_{3}\right) d x_{1} d x_{2} d x_{3},
\end{aligned}
$$

where, also given $\epsilon>0$ and $n_{2} \in \mathbb{N}$, the function $A_{n_{1}, n_{2}, n_{3}}^{\epsilon}: \mathbb{R}^{3 d} \rightarrow[0, \infty)$ is defined by

$$
\begin{gathered}
\left(d\left(n_{1}\right) \Delta_{x_{1}}+d\left(n_{2}\right) \Delta_{x_{2}}+d\left(n_{3}\right) \Delta_{x_{3}}\right) A_{n_{1}, n_{2}, n_{3}}^{\epsilon}\left(x_{1}, x_{2}, x_{3}\right) \\
\quad=-\gamma\left(n_{1}, n_{2}\right) V_{\epsilon}\left(x_{1}-x_{2}\right) J\left(x_{1}, x_{3}\right) .
\end{gathered}
$$

Proof of Lemma 3.1 Let $X(t)$ denote the total number of particles present in the model at time $t$. Then, for any $T>0$,

$$
\mathbb{E}_{N} X(T)=\mathbb{E}_{N} X(0)+\int_{0}^{T} \mathbb{E}_{N} \mathbb{A}_{0} X(t) d t+\int_{0}^{T} \mathbb{E}_{N} \mathbb{A}_{C} X(t) d t
$$

From $A_{0} X=0$, it follows that

$$
-\int_{0}^{T} \mathbb{E}_{N} A_{C} X(t) d t=\mathbb{E}_{N} \int_{0}^{T} d t \sum_{i, j \in I_{q}} \alpha\left(m_{i}, m_{j}\right) V_{\epsilon}\left(x_{i}-x_{j}\right) \leq \mathbb{E}_{N} X(0)=N .
$$

The result follows from the fact that $N=Z \epsilon^{2-d}$.

\section{Proof of Lemma 3.2 Set}

$$
X_{q}=\epsilon^{2(d-2)} \sum_{i, j \in I_{q}} H\left(x_{i}-x_{j}\right) m_{i} m_{j}
$$

Recall the mechanism of the dynamics at collision: the location of the newly created particle is one of the two locations of the colliding particles, with weights proportional to the masses of the 
incident particles. We see that when $\mathbb{A}_{C}$ acts on $X_{q}$, all those terms indexed by pairs of particles one of which is not involved in the collision cancel. Thus,

$$
\mathbb{A}_{C} X=-\epsilon^{2(d-2)} \sum_{i, j \in I_{q}} V_{\epsilon}\left(x_{i}-x_{j}\right) \alpha\left(m_{i}, m_{j}\right) m_{i} m_{j} H\left(x_{i}-x_{j}\right) .
$$

Note also that

$$
\mathbb{A}_{0} X=\epsilon^{2(d-2)} \sum_{i, j \in I_{q}} \Delta H\left(x_{i}-x_{j}\right) m_{i} m_{j}\left(d\left(m_{i}\right)+d\left(m_{j}\right)\right) .
$$

From the non-positivity of $\mathbb{A}_{C} X$, apparent from (3.15), and the non-negativity of $X$, follows

$$
-\mathbb{E}_{N} \int_{0}^{T} \mathbb{A}_{C} X(t) d t-\mathbb{E}_{N} \int_{0}^{T} \mathbb{A}_{0} X(t) d t \leq \mathbb{E}_{N} X(0)
$$

Substituting the form for $\mathbb{A}_{0} X$ and $\mathbb{A}_{C} X$ in (3.16) yields the result.

Proof of Lemma 3.3 Note that

$$
\begin{aligned}
& A_{n_{1}, n_{2}, n_{3}}^{\epsilon}\left(x_{1}, x_{2}, x_{3}\right) \\
&= c_{0}(3 d) \int_{\mathbb{R}^{d}} \int_{\mathbb{R}^{d}} \int_{\mathbb{R}^{d}}\left(\frac{\left|x_{1}-z\right|^{2}}{d\left(n_{1}\right)}+\frac{\left|x_{2}-y\right|^{2}}{d\left(n_{2}\right)}+\frac{\left|x_{3}-y^{\prime}\right|^{2}}{d\left(n_{3}\right)}\right)^{\frac{-3 d+2}{2}} \gamma\left(n_{1}, n_{2}\right) \\
& J\left(z, y^{\prime}\right) V_{\epsilon}(z-y) d z d y d y^{\prime},
\end{aligned}
$$

where $c_{0}$ was defined right after (3.1). Define

$$
X_{n_{1}, n_{2}, n_{3}}(q)=\sum_{i, j, k \in I_{q}} A_{n_{1}, n_{2}, n_{3}}^{\epsilon}\left(x_{i}, x_{j}, x_{k}\right) \mathbb{1}\left\{m_{i}=n_{1}, m_{j}=n_{2}, m_{k}=n_{3}\right\}
$$

and

$$
Y_{n_{1}, n_{3}}(q)=\sum_{n_{2} \in \mathbb{N}} X_{n_{1}, n_{2}, n_{3}}(q)
$$

Note that $\mathbb{A}_{0} Y_{n_{1}, n_{3}}$ is given by

$$
\begin{aligned}
& \sum_{n_{2} \in \mathbb{N}} \sum_{i, j, k \in I_{q}}\left(d\left(n_{1}\right) \Delta_{x_{i}}+d\left(n_{2}\right) \Delta_{x_{j}}+d\left(n_{3}\right) \Delta_{x_{k}}\right) A_{n_{1}, n_{2}, n_{3}}^{\epsilon}\left(x_{i}, x_{j}, x_{k}\right) \\
= & \left.-\sum_{n_{2} \in \mathbb{N}} \sum_{i, j, k \in I_{q}} \gamma\left(m_{1}, n_{2}\right) n_{\epsilon}, m_{j}=n_{2}, m_{k}=x_{j}\right) J\left(x_{i}, x_{k}\right) \mathbb{1}\left\{m_{i}=n_{1}, m_{j}=n_{2}, m_{k}=n_{3}\right\} .
\end{aligned}
$$

Note also that

$$
\mathbb{A}_{C} Y_{n_{1}, n_{3}}=I_{n_{1}, n_{3}}+G_{n_{1}, n_{3}}-L_{n_{1}, n_{3}}
$$

where $I_{n_{1}, n_{3}}$ is given by

$$
\begin{aligned}
& \frac{1}{2} \sum_{k, l \in I_{q}} V_{\epsilon}\left(x_{k}-x_{l}\right) \alpha\left(m_{k}, m_{l}\right) \\
& \sum_{i, j \in I_{q}}\left[\frac{m_{k}}{m_{k}+m_{l}} A_{n_{1}, m_{k}+m_{l}, n_{3}}^{\epsilon}\left(x_{i}, x_{k}, x_{j}\right)+\frac{m_{l}}{m_{k}+m_{l}} A_{n_{1}, m_{k}+m_{l}, n_{3}}^{\epsilon}\left(x_{i}, x_{l}, x_{j}\right)\right. \\
& \left.\quad-A_{n_{1}, m_{k}, n_{3}}^{\epsilon}\left(x_{i}, x_{k}, x_{j}\right)-A_{n_{1}, m_{l}, n_{3}}^{\epsilon}\left(x_{i}, x_{l}, x_{j}\right)\right] \mathbb{1}\left\{m_{i}=n_{1}, m_{j}=n_{3}\right\} .
\end{aligned}
$$


The term $G_{n_{1}, n_{3}}$ is set equal to

$$
\begin{gathered}
\frac{1}{2} \sum_{M_{1}=1}^{n_{1}-1} \sum_{k, l \in I_{q}} V_{\epsilon}\left(x_{k}-x_{l}\right) \alpha\left(m_{k}, m_{l}\right) \mathbb{1}\left\{m_{k}=M_{1}, m_{l}=n_{1}-M_{1}\right\} \\
\sum_{n_{2} \in \mathbb{N}} \sum_{i, j \in I_{q}}\left[\frac{m_{k}}{m_{k}+m_{l}} A_{n_{1}, n_{2}, n_{3}}^{\epsilon}\left(x_{k}, x_{i}, x_{j}\right)\right. \\
\left.+\frac{m_{l}}{m_{k}+m_{l}} A_{n_{1}, n_{2}, n_{3}}^{\epsilon}\left(x_{l}, x_{i}, x_{j}\right)\right] \mathbb{1}\left\{m_{i}=n_{2}, m_{j}=n_{3}\right\} \\
+\frac{1}{2} \sum_{M_{3}=1}^{n_{3}-1} \sum_{k, l \in I_{q}} V_{\epsilon}\left(x_{k}-x_{l}\right) \alpha\left(m_{k}, m_{l}\right) \mathbb{1}\left\{m_{k}=M_{3}, m_{l}=n_{3}-M_{3}\right\} \\
\sum_{n_{2} \in \mathbb{N}} \sum_{i, j \in I_{q}}\left[\frac{m_{k}}{m_{k}+m_{l}} A_{n_{1}, n_{2}, n_{3}}^{\epsilon}\left(x_{i}, x_{j}, x_{k}\right)\right. \\
\left.+\frac{m_{l}}{m_{k}+m_{l}} A_{n_{1}, n_{2}, n_{3}}^{\epsilon}\left(x_{i}, x_{j}, x_{l}\right)\right] \mathbb{1}\left\{m_{i}=n_{1}, m_{j}=n_{2}\right\} .
\end{gathered}
$$

The term $L_{n_{1}, n_{3}}$ may be written as a sum of $L_{n_{1}, n_{3}}^{1}$ and $L_{n_{1}, n_{3}}^{2}$, where $L_{n_{1}, n_{3}}^{1}$ is given by

$$
\begin{aligned}
& \frac{1}{2} \sum_{n_{2} \in \mathbb{N}} \sum_{k, l \in I_{q}} V_{\epsilon}\left(x_{k}-x_{l}\right) \alpha\left(m_{k}, m_{l}\right) \\
& {\left[\sum_{i \in I_{q}} A_{n_{1}, n_{2}, n_{3}}^{\epsilon}\left(x_{i}, x_{k}, x_{l}\right) \mathbb{1}\left\{m_{i}=n_{1}, m_{k}=n_{2}, m_{l}=n_{3}\right\}\right.} \\
& \quad+\sum_{i \in I_{q}} A_{n_{1}, n_{2}, n_{3}}^{\epsilon}\left(x_{k}, x_{i}, x_{l}\right) \mathbb{1}\left\{m_{k}=n_{1}, m_{i}=n_{2}, m_{l}=n_{3}\right\} \\
& \left.\quad+\sum_{i \in I_{q}} A_{n_{1}, n_{2}, n_{3}}^{\epsilon}\left(x_{k}, x_{l}, x_{i}\right) \mathbb{1}\left\{m_{k}=n_{1}, m_{l}=n_{2}, m_{i}=n_{3}\right\}\right],
\end{aligned}
$$

and where $L_{n_{1}, n_{3}}^{2}$ is given by

$$
\begin{aligned}
& \frac{1}{2} \sum_{n_{2} \in \mathbb{N}} \sum_{k, l \in I_{q}} V_{\epsilon}\left(x_{k}-x_{l}\right) \alpha\left(m_{k}, m_{l}\right) \\
& {\left[\sum_{i, j \in I_{q}} A_{n_{1}, n_{2}, n_{3}}^{\epsilon}\left(x_{i}, x_{j}, x_{k}\right) \mathbb{1}\left\{m_{i}=n_{1}, m_{j}=n_{2}, m_{k}=n_{3}\right\}\right.} \\
& \left.\quad+\sum_{i, j \in I_{q}} A_{n_{1}, n_{2}, n_{3}}^{\epsilon}\left(x_{k}, x_{i}, x_{j}\right) \mathbb{1}\left\{m_{k}=n_{1}, m_{i}=n_{2}, m_{j}=n_{3}\right\}\right] .
\end{aligned}
$$

The term $L_{n_{1}, n_{3}}^{1}$ arises due to the loss at collision of particles indexed by $k$ and $l$ in the contribution to $Y_{n_{1}, n_{3}}$ of summands where two of the three arguments of $A^{\epsilon}$ are occupied by $x_{k}$ and $x_{l}$. The term $L_{n_{1}, n_{3}}^{2}$ carries those summands where only one of the pair of particles, $x_{k}$ and $x_{l}$, appears in the arguments of $A^{\epsilon}$. The case where only one of the pair appears, and does so in the unrestricted second argument of $A^{\epsilon}$, is not included here, but instead in the 'internal' term, $I_{n_{1}, n_{3}}$.

By the hypothesis (3.12), we see from the form (3.17) of $A^{\epsilon}$ that $I_{n_{1}, n_{3}} \leq 0$. From (3.18), the lemma will follow provided that we establish the following assertion. 
Claim There exist a collection of constants $C: \mathbb{N}^{2} \rightarrow[0, \infty)$, such that, for each pair $\left(n_{1}, n_{3}\right) \in \mathbb{N}^{2}$, and for all smooth $J: \mathbb{R}^{d} \rightarrow[0, \infty)$, and $\epsilon, T>0$,

$$
\mathbb{E}_{N} \int_{0}^{T} L_{n_{1}, n_{3}}(t) d t \leq R_{n_{1}, n_{3}}, \quad-\mathbb{E}_{N} \int_{0}^{T} \mathbb{A}_{0} Y_{n_{1}, n_{3}}(t) d t \leq R_{n_{1}, n_{3}},
$$

where

$$
R_{n_{1}, n_{3}}=C_{n_{1}, n_{3}} \epsilon^{3(2-d)} \sum_{n_{2} \in \mathbb{N}} \int A_{n_{1}, n_{2}, n_{3}}^{\epsilon}\left(x_{1}, x_{2}, x_{3}\right) h_{n_{1}}\left(x_{1}\right) h_{n_{2}}\left(x_{2}\right) h_{n_{3}}\left(x_{3}\right) d x_{1} d x_{2} d x_{3} .
$$

We establish the claim by an induction on the parameters $n_{1}, n_{3} \in \mathbb{N}$. We proceed to the generic step, at which the claim may be assumed for parameter values $\left(M_{1}, M_{3}\right)$, where $M_{1}<n_{1}$, or $M_{1}=n_{1}, M_{3}<n_{3}$. This step includes the initial case where $n_{1}=n_{3}=1$. From

$$
\mathbb{E}_{N} Y_{n_{1}, n_{3}}(T)=\mathbb{E}_{N} Y_{n_{1}, n_{3}}(0)+\int_{0}^{T} \mathbb{E}_{N}\left(A_{0} Y_{n_{1}, n_{3}}\right)(t) d t+\int_{0}^{T} \mathbb{E}_{N}\left(A_{C} Y_{n_{1}, n_{3}}\right)(t) d t,
$$

and the negativity of the terms $-\mathbb{E}_{N} \int_{0}^{T} L_{n_{1}, n_{3}}(t) d t$ and $\mathbb{E}_{N} \int_{0}^{T} I_{n_{1}, n_{3}}(t) d t$, follows

$$
-\int_{0}^{T} \mathbb{E}_{N}\left(A_{0} Y_{n_{1}, n_{3}}\right)(t) d t \leq \mathbb{E}_{N} Y_{n_{1}, n_{3}}(0)+\mathbb{E}_{N} \int_{0}^{T} G_{n_{1}, n_{3}}(t) d t
$$

Note that

$$
\mathbb{E}_{N} \int_{0}^{T} G_{n_{1}, n_{3}}(t) d t \leq C_{n_{1}} \sum_{M_{1}=1}^{n_{1}-1} \mathbb{E}_{N} \int_{0}^{T} L_{M_{1}, n_{3}}(t) d t+C_{n_{3}} \sum_{M_{3}=1}^{n_{3}-1} \mathbb{E}_{N} \int_{0}^{T} L_{n_{1}, M_{3}}(t) d t
$$

where here we have used such facts as

$$
A_{n_{1}, n_{2}, n_{3}}^{\epsilon} \leq \max \left\{\frac{d\left(n_{1}\right)}{d(j)}, \frac{d(j)}{d\left(n_{1}\right)}\right\}^{\frac{3 d-2}{2}} A_{j, n_{2}, n_{3}}^{\epsilon} \text { for } j \in\left\{1, \ldots, n_{1}-1\right\} .
$$

We do not require any hypotheses on the parameters in this last step, since there are only finitely many possible values of mass for particles that coagulate to form a new particle of some given mass, and we can take care of each of these cases by choosing the value of the constants $C_{n_{1}, n_{3}}$. From (3.23), the inductive hypotheses, the elementary inequalities

$$
\begin{aligned}
& A_{j, n_{2}, n_{3}}^{\epsilon} \leq \max \left\{\frac{d\left(n_{1}\right)}{d(j)}, \frac{d(j)}{d\left(n_{1}\right)}\right\}^{\frac{3 d-2}{2}} A_{n_{1}, n_{2}, n_{3}}^{\epsilon} \text { for } j \in\left\{1, \ldots, n_{1}-1\right\}, \\
& A_{n_{1}, n_{2}, j}^{\epsilon} \leq \max \left\{\frac{d\left(n_{3}\right)}{d(j)}, \frac{d(j)}{d\left(n_{3}\right)}\right\}^{\frac{3 d-2}{2}} A_{n_{1}, n_{2}, n_{3}}^{\epsilon} \text { for } j \in\left\{1, \ldots, n_{3}-1\right\},
\end{aligned}
$$

and the fact that the particles are initially independent, we find that the expression

$$
-\int_{0}^{T} \mathbb{E}_{N}\left(A_{0} Y_{n_{1}, n_{3}}\right)(t) d t
$$

is bounded above by

$$
C_{n_{1}, n_{3}} \epsilon^{3(2-d)} \sum_{n_{2} \in \mathbb{N}} \int A_{n_{1}, n_{2}, n_{3}}^{\epsilon}\left(x_{1}, x_{2}, x_{3}\right) h_{n_{1}}\left(x_{1}\right) h_{n_{2}}\left(x_{2}\right) h_{n_{3}}\left(x_{3}\right) d x_{1} d x_{2} d x_{3},
$$

which is the second part of the claim. To establish the first part, note that

$$
\int_{0}^{T} \mathbb{E}_{N}\left(L_{n_{1}, n_{3}}\right)(t) d t \leq \mathbb{E}_{N} Y_{n_{1}, n_{3}}(0)+\mathbb{E}_{N} \int_{0}^{T} G_{n_{1}, n_{3}}(t) d t
$$


Hence, the term to which the first part of the claim refers satisfies the same upper bound as the one that was just bounded.

The following corollary to Lemma 3.2 will be used in Section 5 .

Lemma 3.4. There exists a constant $C$ such that for any $T \in[0, \infty)$,

$$
\epsilon^{d-2} \mathbb{E}_{N} \int_{0}^{T} d t \sum_{i, j \in I_{q}} m_{i} m_{j} \alpha\left(m_{i}, m_{j}\right) V_{\epsilon}\left(x_{i}-x_{j}\right) \leq C .
$$

Proof On account of Lemma 3.2, it suffices to find a pair of functions $(J, H)=\left(J^{\varepsilon}, H^{\epsilon}\right)$ and a positive constant $\delta$ such that $J \geq 0,-\Delta J=H, \epsilon^{d-2} H^{\epsilon}(x) \geq \delta$ whenever $V_{\epsilon}(x) \neq 0$ and that the expression

$$
X_{N}=\epsilon^{2(d-2)} \mathbb{E}_{N} \sum_{i, j \in I_{q(0)}} H^{\epsilon}\left(x_{i}-x_{j}\right) m_{i} m_{j}
$$

is bounded in $N$. For this, let us pick a nonnegative smooth function $W$ of compact support such that $W(x) \geq 1$ whenever $|x| \leq 2 \eta$ where $\eta$ is chosen so that the support of $V$ is contained in the ball $B_{\eta}(0)$. We then set $J^{\epsilon}(x)=\epsilon^{-d} W(x / \epsilon)$ and $H^{\epsilon}(x)=\epsilon^{2-d} a(x / \epsilon)$ where the function $a$ solves $-\Delta a=W$. More precisely, $a(x)=c_{0}(d) \int W(x-y)|y|^{2-d} d y$. By restricting the domain of $y$-integration to the case $|y| \leq \eta$, it is not hard to deduce that if $|x| \leq \eta$, then $a(x) \geq(d-2)^{-1} \eta$. Also, $a(x) \leq \bar{c}|x|^{2-d}$ for a constant $\bar{c}$. The proof of this is very similar to the proof of the first part of Lemma 3.5 below and omitted. The boundedness of the sequence $\left\{X_{N}\right\}$ is now a straightforward consequence of our assumption $\bar{k} *(-\Delta k)^{-1}(0)<\infty$ on the initial density.

3.2. Bounds on functionals of $u_{n, m}$. We will verify the assertions presented in (3.10). The following lemma provides the bounds on the behaviour of the functions $\left\{u_{n, m}^{\epsilon}: \mathbb{R}^{d} \rightarrow[0, \infty)\right.$ : $(n, m) \in \mathbb{N}\}$ and other functions that will be used in this section. Recall that $u_{n, m}^{\varepsilon}(x)=u^{\varepsilon}(x)=$ $\varepsilon^{2-d} u(x / \varepsilon)$ where $u$ satisfies (1.8). We choose the constant $C_{0}$ so that $V(x)=0$ whenever $|x| \geq C_{0}$. Recall that $k=\sum_{n} n h_{n}$.

Lemma 3.5. There exists a collection of constants $C: \mathbb{N}^{2} \rightarrow(0, \infty)$ for which the following bounds hold.

- for $x \in \mathbb{R}^{d},-u_{n, m}(x) \leq \frac{C_{n, m}}{|x|^{d-2}}$ and $\left|\nabla u_{n, m}(x)\right| \leq \frac{C_{n, m}}{|x|^{d-1}}$.

- for $x \in \mathbb{R}^{d}$ satisfying $|x| \geq \max \left\{2|z|+C_{0} \epsilon, 2 C_{0} \epsilon\right\}$,

$$
\left|u_{n, m}^{\epsilon}(x+z)-u_{n, m}^{\epsilon}(x)\right| \leq \frac{C_{n, m}|z|}{|x|^{d-1}}
$$

and

$$
\left|\nabla u_{n, m}^{\epsilon}(x+z)-\nabla u_{n, m}^{\epsilon}(x)\right| \leq \frac{C_{n, m}|z|}{|x|^{d}} .
$$

- letting $H=H_{n, m}: \mathbb{R}^{d} \rightarrow[0, \infty)$ be given by

$$
-\Delta H_{n, m}(x)=u_{n, m}^{\epsilon}(x+z) \mathbb{1}\{|x| \leq \rho\},
$$


we have

$$
\int H_{n, m}\left(x_{1}-x_{2}\right) k\left(x_{1}\right) k\left(x_{2}\right) d x_{1} d x_{2} \leq C_{n, m}(\rho+|z|)^{2} .
$$

- for $z \in \mathbb{R}^{d}$, let $\hat{H}_{z}\left(=\hat{H}_{z, n, m}\right): \mathbb{R}^{d} \rightarrow[0, \infty)$ be given by

$$
-\Delta \hat{H}_{z}(x)=\left|\nabla u_{n, m}^{\epsilon}(x+z)\right| \mathbb{1}\{|x| \leq \rho\} \text {. }
$$

Then,

$$
\int \hat{H}_{z}\left(x_{1}-x_{2}\right) k\left(x_{1}\right) k\left(x_{2}\right) d x_{1} d x_{2} \leq C_{n, m}(\rho+|z|) .
$$

- for any positive integers $n$ and $m$ and a smooth function $\bar{J}$ of compact support, there exists a constant $C_{n, m}(\bar{J})$ such that for any given $z \in \mathbb{R}^{d}$, the function $A_{n_{1}, n_{2}, n_{3}}^{\epsilon}: \mathbb{R}^{3 d} \rightarrow[0, \infty)$ defined by

$$
\begin{aligned}
& \left(d\left(n_{1}\right) \Delta_{x_{1}}+d\left(n_{2}\right) \Delta_{x_{2}}+d\left(n_{3}\right) \Delta_{x_{3}}\right) A_{n_{1}, n_{2}, n_{3}}^{\epsilon}\left(x_{1}, x_{2}, x_{3}\right) \\
& \quad=-\gamma\left(n_{1}, n_{2}\right) u_{n, m}^{\varepsilon}\left(x_{1}-x_{3}+z\right) \varepsilon^{-2} V\left(\frac{x_{1}-x_{2}}{\epsilon}\right) \mathbb{1}\left(\left|x_{1}-x_{3}\right| \leq \rho\right) \bar{J}\left(x_{3}\right)
\end{aligned}
$$

satisfies

$$
\begin{gathered}
\sum_{n_{1}, n_{2}, n_{3}} \int A_{n_{1}, n_{2}, n_{3}}^{\epsilon}\left(x_{1}, x_{2}, x_{3}\right) h_{n_{1}}\left(x_{1}\right) h_{n_{2}}\left(x_{2}\right) h_{n_{3}}\left(x_{3}\right) d x_{1} d x_{2} d x_{3} \\
\leq C_{n, m}(\bar{J}) \epsilon^{d-2}(\rho+|z|)^{2} .
\end{gathered}
$$

Proof Throughout the proof, we write $u$ for the function $u_{n, m}$ and $\alpha^{\prime}$ for the constant $\alpha^{\prime}(n, m)$ of (2.6). The dependence of the constants on $n$ and $m$ arises from that of $\alpha^{\prime}$, and is also omitted. To prove the first part of the Lemma, we will show that

$$
0 \leq-u(x) \leq c \min \left\{|x|^{2-d}, 1\right\}, \quad 0 \leq|\nabla u(x)| \leq c \min \left\{|x|^{1-d}, 1\right\} .
$$

Note firstly that

$$
u(x)=-c_{0} \alpha^{\prime} \int_{\mathbb{R}^{d}} \frac{V(y)}{|x-y|^{d-2}}[u(y)+1] d y
$$

By Theorem 6.1, $u(x) \in[-1,0]$ for all $x \in \mathbb{R}^{d}$. From this we find that

$$
-u(x) \leq c_{0} \alpha^{\prime} \int_{\mathbb{R}^{d}} \frac{V(y)}{|x-y|^{d-2}} d y
$$

If $|x|$ is large, then $|x-y| \geq|x| / 2$ whenever $V(y) \neq 0$, so that

$$
\int_{\mathbb{R}^{d}} \frac{V(y)}{|x-y|^{d-2}} d y \leq 2^{d-2} \int_{\mathbb{R}^{d}} \frac{V(y)}{|x|^{d-2}} d y=2^{d-2}|x|^{2-d} .
$$

If $|x|$ is small, then

$$
\int_{\mathbb{R}^{d}} \frac{V(y)}{|x-y|^{d-2}} d y \leq \int_{|x-y| \leq C} \frac{V(y)}{|x-y|^{d-2}} d y \leq \int_{0}^{C} \rho d \rho=\frac{C^{2}}{2},
$$

proving the first inequlity in (3.29). In the same way we can deduce the second inequality in (3.29). Indeed

$$
\nabla u(x)=c_{0} \alpha^{\prime}(2-d) \int_{\mathbb{R}^{d}} V(y) \frac{x-y}{|x-y|^{d}}[u(y)+1] d y .
$$


If $|x|$ is large, then $|x-y| \geq|x| / 2$, and

$$
\left|\frac{x-y}{|x-y|^{d}}\right|=\frac{1}{|x-y|^{d-1}} \leq c|x|^{1-d},
$$

whereas, if $|x|$ is small, then

$$
|\nabla u(x)| \leq c \int_{|y| \leq c_{1}} \frac{1}{|x-y|^{d-1}} d y \leq c \int_{0}^{c_{1}} \rho^{d-1} \rho^{1-d} d \rho \leq c_{2} .
$$

Thus,

$$
|\nabla u(x)| \leq c_{0} \min \left\{1,|x|^{1-d}\right\}
$$

and so $|\nabla u(x)| \leq c|x|^{1-d}$.

To prove (3.24) in the second part of the lemma, note that

$$
\begin{aligned}
& \left|u^{\epsilon}(x+z)-u^{\epsilon}(x)\right| \\
\leq & C \int_{\mathbb{R}^{d}} \epsilon^{-d} V\left(\frac{y}{\epsilon}\right)\left|\frac{1}{|x+z-y|^{d-2}}-\frac{1}{|x-y|^{d-2}}\right| d y \\
\leq & C \int_{\mathbb{R}^{d}} \epsilon^{-d} V\left(\frac{y}{\epsilon}\right)\left|\frac{|| x-\left.y\right|^{d-2}-|x+z-y|^{d-2} \mid}{|x+z-y|^{d-2}|x-y|^{d-2}}\right| d y
\end{aligned}
$$

Note that

$$
|x+z-y|^{d-2}-|x-y|^{d-2} \leq(|x-y|+|z|)^{d-2}-|x-y|^{d-2},
$$

and that

$$
|x-y|^{d-2}-|x+z-y|^{d-2} \leq|x-y|^{d-2}-(|x-y|-|z|)^{d-2} .
$$

The right-hand-sides of (3.31) and (3.32) take the form $c \alpha^{d-3}|z|$, for some $\alpha \in[|x-y|-|z|, \mid x-$ $y|+| z \mid]$. Note that if $V(y / \varepsilon) \neq 0$, then

$$
|x-y|-|z| \geq|x|-C_{0} \epsilon-|z| \geq 0,
$$

by our assumption on $|x|$. As a result, we have that $|x-y|+|z| \leq 2|x-y|$, so that

$$
|| x-\left.y\right|^{d-2}-|x+z-y|^{d-2}|\leq C| z|| x-\left.y\right|^{d-3} \text {. }
$$

Hence,

$$
\left|u^{\epsilon}(x+z)-u^{\epsilon}(x)\right| \leq C|z| \int_{\mathbb{R}^{d}} V^{\epsilon}(y) \frac{|x-y|^{-1}}{|x+z-y|^{d-2}} d y .
$$

From the inequality $|x| \geq \max \left\{2|z|+C_{0} \epsilon, 2 C_{0} \epsilon\right\}$, we deduce that $|x+z-y| \geq|x-y| / 2$ and $|x-y| \geq|x| / 2$. We obtain (3.24).

In seeking to prove (3.25), note that

$$
\frac{x+z-y}{|x+z-y|^{d}}-\frac{x-y}{|x-y|^{d}}=\frac{(x+z-y)|x-y|^{d}-(x-y)|x+z-y|^{d}}{|x+z-y|^{d}|x-y|^{d}} \text {. }
$$


Note that, for any $a \in \mathbb{R}^{d}$,

$$
\left.|(a+z)| a\right|^{d}-|a+z|^{d} a|\leq| a|||a+z|^{d}-\left.|a|^{d}|+| z|| a\right|^{d} \leq c_{1}|z||a|^{d},
$$

so long as $|z| \leq|a|$. Given that

$$
\nabla u^{\epsilon}(x)=c_{0}(2-d) \alpha^{\prime} \int_{\mathbb{R}^{d}} V^{\epsilon}(y) \frac{x-y}{|x-y|^{d}}[u(y)+1] d y,
$$

we may apply (3.33) with the choice $a=x-y$ to obtain

$$
\left|\nabla u^{\epsilon}(x+z)-\nabla u^{\epsilon}(x)\right| \leq C|z| \int_{\mathbb{R}^{d}} \frac{V^{\varepsilon}(y)}{|x+z-y|^{d}} d y .
$$

From the inequality $|x| \geq \max \left\{2|z|+C_{0} \epsilon, 2 C_{0} \epsilon\right\}$, we deduce that $|x+z-y| \geq|x-y| / 2$ and $|x-y| \geq|x| / 2$. We conclude that

$$
\left|\nabla u^{\epsilon}(x+z)-\nabla u^{\epsilon}(x)\right| \leq \frac{C|z|}{|x|^{d}}
$$

as required.

To prove the third part of the Lemma, note that

$$
|H(x)| \leq c_{1} \int_{\mathbb{R}^{d}}|x-y|^{2-d}|y+z|^{2-d} \mathbb{1}\{|y| \leq \rho\}
$$

by the first part of the Lemma. Hence,

$$
\begin{aligned}
& \int\left|H\left(x_{1}-x_{2}\right)\right| k\left(x_{1}\right) k\left(x_{2}\right) d x_{1} d x_{2} \\
\leq & c_{1} \int_{|y| \leq \rho}|y+z|^{2-d} \int\left|x_{1}-x_{2}-y\right|^{2-d} k\left(x_{1}\right) k\left(x_{2}\right) d x_{1} d x_{2} d y \\
= & c_{2} \int_{|y| \leq \rho}|y+z|^{2-d}\left(\bar{k} *(-\Delta)^{-1} k\right)(-y) d y \\
\leq & c_{3} \int_{|y| \leq \rho}|y+z|^{2-d} d y \leq c_{4}(\rho+|z|)^{2},
\end{aligned}
$$

where we used our assumption $\bar{k} *(-\Delta)^{-1} k \in L_{l o c}^{\infty}$ for the third inequality. This establishes the third part of the Lemma.

To prove the fourth part of the Lemma, we firstly find a bound for $\nabla u^{\epsilon}(x)=\epsilon^{1-d} \nabla u(x / \epsilon)$. Using the first part of the Lemma,

$$
\hat{H}_{z}(x) \leq c \int_{\mathbb{R}^{d}}|x-y|^{2-d}|y+z|^{1-d} \mathbb{1}\{|y| \leq \rho\}
$$

it follows that

$$
\begin{aligned}
& \int\left|\hat{H}_{z}\left(x_{1}-x_{2}\right)\right| k\left(x_{1}\right) k\left(x_{2}\right) d x_{1} d x_{2} \\
\leq & c_{1} \int_{|y| \leq \rho}|y+z|^{1-d} \int\left|x_{1}-x_{2}-y\right|^{2-d} k\left(x_{1}\right) k\left(x_{2}\right) d x_{1} d x_{2} d y \\
\leq & c_{2} \int_{|y| \leq \rho}|y+z|^{1-d} d y \leq c_{3}(\rho+|z|) .
\end{aligned}
$$


We have deduced (3.26) .

As for the fifth part of the Lemma, let us write $J(a)$ for $\mathbb{1}(|a| \leq \rho)$ and define the quantity $I$ to be

$$
\begin{gathered}
c_{0}(3 d) \sum_{n_{1}, n_{2}, n_{3}} \int d x_{1} d x_{2} d x_{3} \int_{\mathbb{R}^{3 d}} \gamma\left(n_{1}, n_{2}\right)\left(\frac{\left|x_{1}-z^{\prime}\right|^{2}}{d\left(n_{1}\right)}+\frac{\left|x_{2}-y\right|^{2}}{d\left(n_{2}\right)}+\frac{\left|x_{3}-y^{\prime}\right|^{2}}{d\left(n_{3}\right)}\right)^{\frac{-3 d+2}{2}} \\
h_{n_{1}}\left(x_{1}\right) h_{n_{2}}\left(x_{2}\right) h_{n_{3}}\left(x_{3}\right) u^{\varepsilon}\left(z^{\prime}-y^{\prime}+z\right) \varepsilon^{-2} V\left(\frac{z^{\prime}-y}{\epsilon}\right) J\left(z^{\prime}-y^{\prime}\right) \bar{J}\left(y^{\prime}\right) d z^{\prime} d y d y^{\prime} .
\end{gathered}
$$

We write

$$
I=c_{0}(3 d) \int_{\mathbb{R}^{3 d}} u^{\varepsilon}\left(z^{\prime}-y^{\prime}+z\right) \varepsilon^{-2} V\left(\frac{z^{\prime}-y}{\epsilon}\right) J\left(z^{\prime}-y^{\prime}\right) \bar{J}\left(y^{\prime}\right) G\left(z^{\prime}, y, y^{\prime}\right) d z^{\prime} d y d y^{\prime},
$$

where $G\left(z^{\prime}, y, y^{\prime}\right)$ is given by

$$
\sum_{n_{1}, n_{2}, n_{3}} \gamma\left(n_{1}, n_{2}\right) \int\left(\frac{\left|x_{1}-z^{\prime}\right|^{2}}{d\left(n_{1}\right)}+\frac{\left|x_{2}-y\right|^{2}}{d\left(n_{2}\right)}+\frac{\left|x_{3}-y^{\prime}\right|^{2}}{d\left(n_{3}\right)}\right)^{\frac{-3 d+2}{2}} h_{n_{1}}\left(x_{1}\right) h_{n_{2}}\left(x_{2}\right) h_{n_{3}}\left(x_{3}\right) d x_{1} d x_{2} d x_{3} .
$$

Using the elementary inequality $a b c \leq\left(a^{2}+b^{2}+c^{2}\right)^{3 / 2}$ we deduce that $G\left(z^{\prime}, y, y^{\prime}\right)$ is at most

$$
\begin{gathered}
\sum_{n_{1}, n_{2}, n_{3}} \gamma\left(n_{1}, n_{2}\right)\left(d\left(n_{1}\right) d\left(n_{2}\right) d\left(n_{3}\right)\right)^{d / 2-1 / 3} \int\left|x_{1}-z^{\prime}\right|^{-d+2 / 3}\left|x_{2}-y\right|^{-d+2 / 3}\left|x_{3}-y^{\prime}\right|^{-d+2 / 3} \\
h_{n_{1}}\left(x_{1}\right) h_{n_{2}}\left(x_{2}\right) h_{n_{3}}\left(x_{3}\right) d x_{1} d x_{2} d x_{3} .
\end{gathered}
$$

From our asumptions on $h_{n}$ we deduce that $G \in L_{l o c}^{\infty}$. Hence,

$$
I \leq C \int_{\mathbb{R}^{3 d}} u^{\varepsilon}\left(z^{\prime}-y^{\prime}+z\right) \varepsilon^{-2} V\left(\frac{z^{\prime}-y}{\epsilon}\right) J\left(z^{\prime}-y^{\prime}\right) \bar{J}\left(y^{\prime}\right) d z^{\prime} d y d y^{\prime} .
$$

Note that, for fixed $\left(z^{\prime}, y^{\prime}\right) \in \mathbb{R}^{2 d}$,

$$
\int_{\mathbb{R}^{d}} V\left(\frac{z^{\prime}-y}{\epsilon}\right) d y=\epsilon^{d}
$$

Thus,

$$
\begin{aligned}
I & \leq C \epsilon^{d-2} \int_{\mathbb{R}^{2 d}} u^{\varepsilon}\left(z^{\prime}-y^{\prime}+z\right) J\left(z^{\prime}-y^{\prime}\right) \bar{J}\left(y^{\prime}\right) d z^{\prime} d y^{\prime} \\
& \leq C \epsilon^{d-2} \int_{K} \int u^{\varepsilon}\left(z^{\prime}-y^{\prime}+z\right) J\left(z^{\prime}-y^{\prime}\right) d z^{\prime} d y^{\prime} \\
& \leq C \epsilon^{d-2} \int_{K} d y^{\prime} \int \frac{1}{\left|z^{\prime}-y^{\prime}+z\right|^{d-2}} J\left(z^{\prime}-y^{\prime}\right) d z^{\prime} \\
& \leq C \epsilon^{d-2} \int_{|a| \leq \rho+|z|} \frac{1}{|a|^{d-2}} d a \leq C \epsilon^{d-2}(\rho+|z|)^{2},
\end{aligned}
$$

where $K \subseteq \mathbb{R}^{d}$ denotes a compact set containing the support $\bar{J}$, and where we made use of the first part of the Lemma in the third inequality. This is the bound stated in (3.28).

\subsection{Estimating the terms.}


3.3.1. The case of $H_{14}$. Note that

$$
\begin{gathered}
\mathbb{E}_{N}\left|\int_{0}^{T} H_{14}(t) d t\right| \leq C \epsilon^{2(d-2)} \mathbb{E}_{N} \int_{0}^{T} \sum_{i, j \in I_{q}}\left|u^{\epsilon}\left(x_{i}-x_{j}+z\right)-u^{\epsilon}\left(x_{i}-x_{j}\right)\right| \\
\mathbb{1}\left\{m_{i}=M_{1}, m_{j}=M_{2}\right\},
\end{gathered}
$$

where the constant $C$ depends on the $L^{\infty}$ bounds satisifed by $J, \bar{J}$ and their time derivatives. Hence

$$
\mathbb{E}_{N}\left|\int_{0}^{T} H_{14}(t) d t\right| \leq K_{1}+K_{2}
$$

where $K_{1}$ is given by

$$
C \epsilon^{2(d-2)} \mathbb{E}_{N} \int_{0}^{T} d t \sum_{i, j \in I_{q}:\left|x_{i}-x_{j}\right|>\rho}\left|u^{\epsilon}\left(x_{i}-x_{j}+z\right)-u^{\epsilon}\left(x_{i}-x_{j}\right)\right| \mathbb{1}\left\{m_{i}=M_{1}\right\} \mathbb{1}\left\{m_{j}=M_{2}\right\}
$$

and $K_{2}$ is given by

$$
\epsilon^{2(d-2)} \mathbb{E}_{N} \int_{0}^{T} d t \sum_{i, j \in I_{q}:\left|x_{i}-x_{j}\right| \leq \rho}\left|u^{\epsilon}\left(x_{i}-x_{j}+z\right)-u^{\epsilon}\left(x_{i}-x_{j}\right)\right| \mathbb{1}\left\{m_{i}=M_{1}\right\} \mathbb{1}\left\{m_{j}=M_{2}\right\} .
$$

Firstly, we treat $K_{1}$. Note that

$$
\begin{aligned}
& \leq \frac{C|z| \epsilon^{2(d-2)}}{\rho^{d-1}} \mathbb{E}_{N} \int_{0}^{T} d t \sum_{i, j \in I_{q}:\left|x_{i}-x_{j}\right|>\rho} \mathbb{1}\left\{m_{i}=M_{1}\right\} \mathbb{1}\left\{m_{j}=M_{2}\right\} \\
& \leq \frac{C|z|}{\rho^{d-1}} \epsilon^{2(d-2)} \mathbb{E}_{N} \int_{0}^{T} d t \sum_{i, j \in I_{q}:\left|x_{i}-x_{j}\right|>\rho} m_{i} m_{j} \leq \frac{C|z| T}{\rho^{d-1}}\left(\epsilon^{d-2} \sum_{i \in I_{q(0)}} m_{i}\right)^{2} \leq \frac{C|z| T}{\rho^{d-1}},
\end{aligned}
$$

where the first inequality follows from the second part of Lemma 3.5, and the final one from an hypothesis on the initial conditions of the system. We now treat the term $K_{2}$. By writing,

$$
\begin{gathered}
K_{2} \leq C \epsilon^{2(d-2)} \mathbb{E}_{N} \int_{0}^{T} d t \sum_{\substack{i, j \in I_{q}:\left|x_{i}-x_{j}\right| \leq \rho \\
1}}\left[u^{\epsilon}\left(x_{i}-x_{j}+z\right)+u^{\epsilon}\left(x_{i}-x_{j}\right)\right] \\
\mathbb{1}\left\{m_{i}=M_{1}\right\} \mathbb{1}\left\{m_{j}=M_{2}\right\},
\end{gathered}
$$

we obtain an expression on the right-hand-side which may be bounded by applying Lemma 3.2 . The relevant estimate is provided by the third part of Lemma 3.5. We find that $K_{2} \leq C(\rho+|z|)^{2}$. Hence,

$$
\mathbb{E}_{N}\left|\int_{0}^{T} H_{14}(t)\right| d t \leq K_{1}+K_{2} \leq \frac{C|z| T}{\rho^{d-1}}+C(\rho+|z|)^{2}
$$

Making the choice $\rho=|z|^{\frac{1}{d+1}}$, we find that

$$
\mathbb{E}_{N}\left|\int_{0}^{T} H_{14}(t)\right| d t \leq C|z|^{\frac{2}{d+1}}
$$


3.3.2. The cases of $H_{2}$ and $H_{3}$. The estimate of $\mathbb{E}_{N} \int_{0}^{T}\left|H_{3}(t)\right| d t$ is derived in an identical fashion to that of $\mathbb{E}_{N} \int_{0}^{T}\left|H_{2}(t)\right| d t$. Picking $\rho \in \mathbb{R}$ that satisfies $\rho \geq \max \left\{2|z|+C_{0} \epsilon, 2 C_{0} \epsilon\right\}$, we write

$$
\int_{0}^{T} \mathbb{E}_{N}\left|H_{2}(t)\right| d t \leq R_{1}+R_{2}
$$

where

$$
\begin{array}{r}
R_{1}=2 \epsilon^{2(d-2)} \mathbb{E}_{N} \int_{0}^{T} \sum_{i, j \in I_{q}:\left|x_{i}-x_{j}\right|>\rho} d\left(m_{i}\right)\left|\bar{J}\left(x_{j}, m_{j}, t\right)\right| \\
\left|u_{x}^{\epsilon}\left(x_{i}-x_{j}+z\right)-u_{x}^{\epsilon}\left(x_{i}-x_{j}\right)\right|\left|J_{x}\left(x_{i}, m_{i}, t\right)\right| d t,
\end{array}
$$

and

$$
\begin{array}{r}
R_{2}=2 \epsilon^{2(d-2)} \mathbb{E}_{N} \int_{0}^{T} \sum_{i, j \in I_{q}:\left|x_{i}-x_{j}\right| \leq \rho} d\left(m_{i}\right)\left|\bar{J}\left(x_{j}, m_{j}, t\right)\right| \\
\left|u_{x}^{\epsilon}\left(x_{i}-x_{j}+z\right)-u_{x}^{\epsilon}\left(x_{i}-x_{j}\right)\right|\left|J_{x}\left(x_{i}, m_{i}, t\right)\right| d t .
\end{array}
$$

Firstly, we examine the sum $R_{1}$. Recalling that we consider test functions $J$ and $\bar{J}$ respectively supported on particles of mass $M_{1}$ and $M_{2}$,

$$
R_{1} \leq C \epsilon^{2(d-2)} \frac{|z|}{\rho^{d}} d\left(M_{1}\right)\left|\left\{(i, j) \in I_{q}^{2}: m_{i}=M_{1}, m_{j}=M_{2}\right\}\right|,
$$

where the lower bound on $\rho$ allowed us to apply the second part of Lemma 3.5. Thus, $R_{1} \leq C|z| / \rho^{d}$.

Secondly, we bound the sum $R_{2}$. Note that

$$
\begin{gathered}
R_{2} \leq 2 \epsilon^{2(d-2)}\left\|J_{x}\right\|\|\bar{J}\| d\left(M_{1}\right) \mathbb{E}_{N} \int_{0}^{T} \sum_{i, j \in I_{q}:\left|x_{i}-x_{j}\right| \leq \rho} \\
\left|u_{x}^{\epsilon}\left(x_{i}-x_{j}+z\right)-u_{x}^{\epsilon}\left(x_{i}-x_{j}\right)\right| \mathbb{1}\left\{m_{i}=M_{1}, m_{j}=M_{2}\right\} \\
\leq C \epsilon^{2(d-2)} \mathbb{E}_{N} \int_{0}^{T} \sum_{i, j \in I_{q}} m_{i} m_{j}\left(d\left(m_{i}\right)+d\left(m_{j}\right)\right) \\
{\left[\left|u_{x}^{\epsilon}\left(x_{i}-x_{j}+z\right)\right|+\left|u_{x}^{\epsilon}\left(x_{i}-x_{j}\right)\right|\right]}
\end{gathered}
$$

where $\|\cdot\|$ denotes the $L^{\infty}$ norm and the constant $C$ depends on the test functions $J$ and $\bar{J}$. The expression (3.44) is written in a form to which Lemma 3.2 may be applied. Doing so yields

$$
R_{2} \leq C \epsilon^{2(d-2)} \mathbb{E}_{N} \sum_{i, j \in I_{q}} m_{i} m_{j}\left(\hat{H}_{0}\left(x_{i}-x_{j}\right)+\hat{H}_{z}\left(x_{i}-x_{j}\right)\right),
$$

where the functions $\left\{\hat{H}_{z}: z \in \mathbb{R}^{d}\right\}$ appear in the fourth part of Lemma 3.5. From that result and our assumptions on the initial data, we deduce that $R_{2} \leq C(\rho+|z|)$. Hence,

$$
\int_{0}^{T} \mathbb{E}_{N}\left|H_{2}(t)\right| d t \leq R_{1}+R_{2} \leq C \frac{|z|}{\rho^{d}}+C(\rho+|z|),
$$


for $\rho \leq 1$. By making the choice $\rho=|z|^{\frac{1}{d+1}}$, we find that

$$
\int_{0}^{T} \mathbb{E}_{N}\left|H_{2}(t)\right| d t \leq C|z|^{\frac{1}{d+1}}
$$

3.3.3. The case of $\mathrm{H}_{4}$. Note that

$$
\begin{array}{ll}
\int_{0}^{T} \mathbb{E}_{N}\left|H_{4}(t)\right| d t \leq & \left(d\left(M_{1}\right)\|\Delta J\|\|\bar{J}\|+d\left(M_{2}\right)\|J\|\|\Delta \bar{J}\|\right) \epsilon^{2(d-2)} \\
& \quad \mathbb{E}_{N} \int_{0}^{T} d t \sum_{i, j \in I_{q}} \mathbb{1}\left\{m_{i}=M_{1}, m_{j}=M_{2}\right\}\left|u^{\epsilon}\left(x_{i}-x_{j}+z\right)-u^{\epsilon}\left(x_{i}-x_{j}\right)\right| .
\end{array}
$$

This quantity is bounded as in the case of $H_{14}$.

3.3.4. The case of $G_{z}(1)-G_{0}(1)$. We now estimate the term

$$
\int_{0}^{T} \mathbb{E}_{N}\left|G_{z}(1)-G_{0}(1)\right|(t) d t
$$

To ease the notation, we do not display the dependece of $J$ and $\bar{J}$ on the variable $t$. Note that

$$
\int_{0}^{T} \mathbb{E}_{N}\left|G_{z}(1)-G_{0}(1)\right|(t) d t \leq \sum_{i=1}^{8} D_{i}
$$

where

$$
\begin{aligned}
D_{1}=\frac{1}{2} \mathbb{E}_{N} \int_{0}^{T} d t \sum_{k, l \in I_{q}} \alpha\left(m_{k}, m_{l}\right) V_{\epsilon}\left(x_{k}-x_{l}\right)\left|J\left(x_{k}, m_{k}\right)\right| \\
\epsilon^{2(d-2)} \sum_{i \in I_{q}}\left|\bar{J}\left(x_{i}, m_{i}\right)\right|\left|u^{\epsilon}\left(x_{k}-x_{i}+z\right)-u^{\epsilon}\left(x_{k}-x_{i}\right)\right|,
\end{aligned}
$$

each of the other seven terms on the right-hand-side of (3.46) differing from $D_{1}$ only in an inessential way. Given this. the estimates involved for each of the eight cases are in essence identical, and we examine only the case of $D_{1}$. We write $D_{1}=D^{1}+D^{2}$, where we have decomposed the inner $i$-indexed sum according to the respective index sets

$$
\left\{i \in I_{q}, i \neq k, l,\left|x_{k}-x_{i}\right|>\rho\right\} \text { and }\left\{i \in I_{q}, i \neq k, l,\left|x_{k}-x_{i}\right| \leq \rho\right\} .
$$

Here, $\rho$ is a positive parameter that satisfies the bound $\rho \geq \max \left\{2|z|+C_{0} \epsilon, 2 C_{0} \epsilon\right\}$. By the second part of Lemma 3.5, we have that

$$
D^{1} \leq \frac{C|z| \epsilon^{d-2}}{\rho^{d-1}} \mathbb{E}_{N} \int_{0}^{T} d t \sum_{k, l \in I_{q}} \alpha\left(m_{k}, m_{l}\right) V_{\epsilon}\left(x_{k}-x_{l}\right),
$$

where we have also used the fact that the test functions $J$ and $\bar{J}$ are each supported on the set of particles of respective masses $M_{1}$ and $M_{2}$, and the fact that the total number of particles living at any given time is bounded above by $Z \epsilon^{2-d}$. From the bound on the collision that is provided by Lemma 3.1, follows

$$
D^{1} \leq \frac{C|z|}{\rho^{d-1}}
$$


To bound the term $D^{2}$, note that $D^{2}$ is bounded above by

$$
\begin{gathered}
C \mathbb{E}_{N} \int_{0}^{T} d t \sum_{k, l \in I_{q}} \alpha\left(m_{k}, m_{l}\right) V_{\epsilon}\left(x_{k}-x_{l}\right) \mathbb{1}\left\{m_{k}=M_{1}\right\} \epsilon^{2(d-2)} \sum_{i \in I_{q}} \\
\mathbb{1}\left\{\left|x_{i}-x_{k}\right| \leq \rho\right\} \mathbb{1}\left\{m_{i}=M_{2}\right\}\left|u^{\epsilon}\left(x_{k}-x_{i}+z\right)-u^{\epsilon}\left(x_{k}-x_{i}\right)\right|\left|J\left(x_{i}, m_{i}\right)\right| \\
\leq C \mathbb{E}_{N} \int_{0}^{T} d t \sum_{k, l \in I_{q}} \alpha\left(m_{k}, m_{l}\right) V_{\epsilon}\left(x_{k}-x_{l}\right) \mathbb{1}\left\{m_{k}=M_{1}\right\} \epsilon^{2(d-2)} \sum_{i \in I_{q}} \\
\mathbb{1}\left\{\left|x_{i}-x_{k}\right| \leq \rho\right\} \mathbb{1}\left\{m_{i}=M_{2}\right\}\left[\left|u^{\epsilon}\left(x_{k}-x_{i}+z\right)\right|+\left|u^{\epsilon}\left(x_{k}-x_{i}\right)\right|\right]\left|J\left(x_{i}, m_{i}\right)\right| .
\end{gathered}
$$

Note that the last expectation is bounded by Lemma 3.3 because by our assumption on $\alpha$, we can find $\gamma$ such that $\alpha \leq \gamma$ and $\gamma$ satisfies the assumption of Lemma 3.3. The upper bound provided by this Lemma in this particular application is computed in the fifth part of Lemma 3.5. We find that $D^{2} \leq C(\rho+|z|)^{2}$.

Combining these estimates yields

$$
D_{1} \leq D^{1}+D^{2} \leq C \frac{|z|}{\rho^{d-1}}+C(\rho+|z|)^{2} .
$$

Making the choice $\rho=|z|^{\frac{1}{d+1}}$ leads to the inequality $D_{1} \leq|z|^{\frac{2}{d+1}}$. Since each of the cases of $\left\{D_{i}: i \in\{1, \ldots, 8\}\right\}$ may be treated by a nearly verbatim proof, we deduce that

$$
\int_{0}^{T} \mathbb{E}_{N}\left|G_{z}(1)-G_{0}(1)\right|(t) d t \leq C|z|^{\frac{2}{d+1}} .
$$

3.3.5. The case of $G_{z}(2)$. Recall that

$$
G_{z}(2)=-\epsilon^{2(d-2)} \sum_{k, l \in I_{q}} \alpha\left(m_{k}, m_{l}\right) V_{\epsilon}\left(x_{k}-x_{l}\right) u^{\epsilon}\left(x_{k}-x_{l}+z\right) J\left(x_{k}, m_{k}\right) \bar{J}\left(x_{l}, m_{l}\right) .
$$

If $k, l \in I_{q}$ satisfy $V_{\epsilon}\left(x_{k}-x_{l}\right) \neq 0$, then $\left|x_{k}-x_{l}\right| \leq C_{0} \epsilon$, and so

$$
\left|x_{k}-x_{l}+z\right| \geq|z|-C_{0} \epsilon \geq|z| / 2
$$

provided that $|z| \geq 2 C_{0} \varepsilon$. This implies that

$$
u^{\epsilon}\left(x_{k}-x_{l}+z\right) \leq \frac{C}{\left|x_{k}-x_{l}+z\right|^{d-2}} \leq \frac{C}{|z|^{d-2}},
$$

where in the first inequality, we used the first part of Lemma 3.5. Applying this bound, we find that

$$
\int_{0}^{T} \mathbb{E}_{N}\left|G_{z}(2)\right| d t \leq \frac{C \epsilon^{2(d-2)}}{|z|^{d-2}} \mathbb{E}_{N} \int_{0}^{T} \sum_{k, l \in I_{q}} \alpha\left(m_{k}, m_{l}\right) V_{\epsilon}\left(x_{k}-x_{l}\right) d t
$$

whose right-hand-side is bounded above by $C \epsilon^{d-2} /|z|^{d-2}$, according to Lemma 3.1. As a result,

$$
\int_{0}^{T}\left|G_{z}(2)\right| d t \leq C\left(\frac{\epsilon}{|z|}\right)^{d-2}
$$


3.3.6. The case of $\mathbb{E}_{N}\left|X_{z}-X_{0}\right|$. We now turn to $\mathbb{E}_{N}\left|X_{z}-X_{0}\right|$. Assume that $|z| \geq C_{0} \varepsilon$. Using the first and second part of Lemma 3.5 we have that

$$
\begin{aligned}
\mathbb{E}_{N}\left|X_{z}-X_{0}(0)\right| \leq \quad & C|z| \iint_{L^{2}} h_{M_{1}}(x) h_{M_{2}}(y)|x-y|^{1-d} d x d y \\
& +C \iint_{L^{2}} h_{M_{1}}(x) h_{M_{2}}(y)|x-y|^{2-d} \mathbb{1}(|x-y| \leq 3|z|) d x d y \\
& +C \iint_{L^{2}} h_{M_{1}}(x) h_{M_{2}}(y)|x-y+z|^{2-d} \mathbb{1}(|x-y+z| \leq 4|z|) d x d y
\end{aligned}
$$

where $L$ is a bounded set that contains the support of $J$ and $\bar{J}$. Using our assumptions on the initial data $h_{n}$ we obtain the bound $C|z|$ for the first term and $C|z|^{2 / 3}$ for the other two terms on the right-hand-side. This evidently implies our bound on $\mathbb{E}_{N}\left|X_{z}-X_{0}\right|(0)$ in (3.10).

3.4. The martingale term. This section is devoted to proving the estimate (3.11). Note that

$$
\left.M_{z}(T)=X_{z}(q(T), T)-X_{z}(q(0), 0)-\int_{0}^{T}\left(\frac{\partial}{\partial t}+\mathbb{L}\right) X_{z}(q(t), t)\right) d t
$$

is a martingale which satisfies

$$
\mathbb{E}_{N}\left[M_{z}(T)^{2}\right]=\sum_{i=1}^{3} \mathbb{E}_{N} \int_{0}^{T} A_{i}(q(t), t) d t
$$

where $A_{1}(q, t)$ is set equal to

$$
2 \epsilon^{4(d-2)} \sum_{i \in I_{q}, m_{i}=M_{1}} d\left(M_{1}\right)\left[\nabla_{x_{i}} \sum_{j \in I_{q}, m_{j}=M_{2}} u^{\epsilon}\left(x_{i}-x_{j}+z\right) J\left(x_{i}, M_{1}, t\right) \bar{J}\left(x_{j}, M_{2}, t\right)\right]^{2},
$$

with $A_{2}$ being given by the same expression with instances of $M_{1}$ and $M_{2}$ being interchanged, including those implicitly appearing in the function $u^{\epsilon}=u_{M_{1}, M_{2}}^{\epsilon}$. The term $A_{3}$ is given by

$$
\begin{aligned}
& \frac{1}{2} \epsilon^{4(d-2)} \sum_{i, j \in I_{q}} \alpha\left(m_{i}, m_{j}\right) V_{\epsilon}\left(x_{i}-x_{j}\right) \\
& \left\{\sum _ { k \in I _ { q } } \left[\frac{m_{i}}{m_{i}+m_{j}} u^{\epsilon}\left(x_{i}-x_{k}+z\right) J\left(x_{i}, m_{i}+m_{j}\right) \bar{J}\left(x_{k}, m_{k}\right)\right.\right. \\
& +\frac{m_{i}}{m_{i}+m_{j}} u^{\epsilon}\left(x_{k}-x_{i}+z\right) J\left(x_{k}, m_{k}\right) \bar{J}\left(x_{i}, m_{i}+m_{j}\right) \\
& +\frac{m_{j}}{m_{i}+m_{j}} u^{\epsilon}\left(x_{j}-x_{k}+z\right) J\left(x_{j}, m_{i}+m_{j}\right) \bar{J}\left(x_{k}, m_{k}\right) \\
& +\frac{m_{j}}{m_{i}+m_{j}} u^{\epsilon}\left(x_{k}-x_{j}+z\right) J\left(x_{k}, m_{k}\right) \bar{J}\left(x_{j}, m_{i}+m_{j}\right) \\
& -u^{\epsilon}\left(x_{i}-x_{k}+z\right) J\left(x_{i}, m_{i}\right) \bar{J}\left(x_{k}, m_{k}\right)-u^{\epsilon}\left(x_{k}-x_{i}+z\right) J\left(x_{k}, m_{k}\right) \bar{J}\left(x_{i}, m_{i}\right) \\
& \left.-u^{\epsilon}\left(x_{j}-x_{k}+z\right) J\left(x_{j}, m_{j}\right) \bar{J}\left(x_{k}, m_{k}\right)-u^{\epsilon}\left(x_{k}-x_{j}+z\right) J\left(x_{k}, m_{k}\right) \bar{J}\left(x_{j}, m_{j}\right)\right] \\
& \left.-u^{\epsilon}\left(x_{i}-x_{j}+z\right) J\left(x_{i}, m_{i}\right) \bar{J}\left(x_{j}, m_{j}\right)\right\}^{2} .
\end{aligned}
$$


Recall that we by our convention, we do not display the dependence of $J$ and $\bar{J}$ on the $t$-variable. To bound these terms, we require two variants of Lemma 3.3.

Lemma 3.6. There exists a collection of constants $C: \mathbb{N}^{2} \rightarrow(0, \infty)$ such that, for any continuous functions $t, v, a_{1}, a_{2}, a_{3}: \mathbb{R}^{d} \rightarrow[0, \infty)$ and any $z \in \mathbb{R}^{d}$,

$$
\begin{aligned}
\mathbb{E}_{N} \int_{0}^{T} d t & \sum_{i, j, k \in I_{q}(t)} \gamma\left(m_{i}, m_{j}\right) t\left(\frac{x_{i}-x_{j}+z}{\epsilon}\right) v\left(\frac{x_{i}-x_{k}+z}{\epsilon}\right) \\
& a_{1}\left(x_{i}\right) a_{2}\left(x_{j}\right) a_{3}\left(x_{k}\right) \mathbb{1}\left\{m_{i}=n_{1}, m_{k}=n_{3}\right\} \\
\leq & C_{n_{1}, n_{3}} \epsilon^{3(2-d)} \sum_{n_{2}} \mathbb{E}_{N} \sum_{i, j, k \in I_{q(0)}} A_{n_{1}, n_{2}, n_{3}}^{\epsilon}\left(x_{i}, x_{j}, x_{k}\right),
\end{aligned}
$$

where $A_{n_{1}, n_{2}, n_{3}}^{\epsilon}: \mathbb{R}^{3 d} \rightarrow[0, \infty)$ is given by

$$
\begin{aligned}
A_{n_{1}, n_{2}, n_{3}}^{\epsilon}\left(x_{1}, x_{2}, x_{3}\right)= & c_{0}(3 d) \gamma\left(n_{1}, n_{2}\right) \int_{\mathbb{R}^{3 d}}\left(\frac{\left|x_{1}-z^{\prime}\right|^{2}}{d\left(n_{1}\right)}+\frac{\left|x_{2}-y\right|^{2}}{d\left(n_{2}\right)}+\frac{\left|x_{3}-y^{\prime}\right|^{2}}{d\left(n_{3}\right)}\right)^{\frac{-3 d+2}{2}} \\
& t\left(\frac{z^{\prime}-y+z}{\epsilon}\right) v\left(\frac{z^{\prime}-y^{\prime}+z}{\epsilon}\right) a_{1}\left(z^{\prime}\right) a_{2}(y) a_{3}\left(y^{\prime}\right) d z^{\prime} d y d y
\end{aligned}
$$

with $\gamma$ as in Lemma 3.3.

Lemma 3.7. There exists a collection of constants $C: \mathbb{N}^{3} \rightarrow[0, \infty)$ such that, for any $z \in \mathbb{R}^{d}$, any continuous functions $v, w: \mathbb{R}^{d} \rightarrow[0, \infty)$ and another $\left(a_{1}, a_{2}, a_{3}\right): \mathbb{R}^{3 d} \rightarrow[0, \infty)$,

$$
\begin{gathered}
\mathbb{E}_{N} \int_{0}^{T} d t \sum_{k, l, i, j \in I_{q}} \gamma\left(n_{i}, n_{j}\right) V_{\varepsilon}\left(x_{i}-x_{j}\right) v\left(\frac{x_{i}-x_{k}+z}{\epsilon}\right) w\left(\frac{x_{i}-x_{l}+z}{\epsilon}\right) a_{1}\left(x_{i}\right) a_{2}\left(x_{k}\right) a_{3}\left(x_{l}\right) \\
\mathbb{1}\left\{m_{i}=n_{1}, m_{k}=n_{3}, m_{l}=n_{4}\right\} \\
\leq C_{n_{1}, n_{3}, n_{4}} \epsilon^{4(2-d)} \sum_{n_{2}} \mathbb{E}_{N} \sum_{i, j, k, l \in I_{q(0)}} B_{m_{i}, m_{j}, m_{k}, m_{l}}^{\epsilon}\left(x_{i}, x_{j}, x_{k}, x_{l}\right) \\
\mathbb{1}\left\{m_{i} \leq n_{1}, m_{j} \leq n_{2}, m_{k} \leq n_{3}, m_{l} \leq n_{4}\right\}
\end{gathered}
$$

where $B_{n_{1}, n_{2}, n_{3}, n_{4}}^{\epsilon}: \mathbb{R}^{4 d} \rightarrow[0, \infty)$ is given by

$$
\begin{aligned}
& B_{n_{1}, n_{2}, n_{3}, n_{4}}^{\epsilon}\left(x_{1}, x_{2}, x_{3}, x_{4}\right) \\
= & \int_{\mathbb{R}^{4 d}}\left(\frac{\left|x_{1}-\hat{z}\right|^{2}}{d\left(n_{1}\right)}+\frac{\left|x_{2}-z^{\prime}\right|^{2}}{d\left(n_{2}\right)}+\frac{\left|x_{3}-y\right|^{2}}{d\left(n_{3}\right)}+\frac{\left|x_{4}-y^{\prime}\right|^{2}}{d\left(n_{4}\right)}\right)^{-2 d+1} \\
& \gamma\left(n_{1}, n_{2}\right) V_{\varepsilon}\left(\hat{z}-z^{\prime}\right) v\left(\frac{\hat{z}-y+z}{\epsilon}\right) w\left(\frac{z^{\prime}-y^{\prime}+z}{\epsilon}\right) a_{1}(\hat{z}) a_{2}(y) a_{3}\left(y^{\prime}\right) d \hat{z} d z^{\prime} d y d y^{\prime},
\end{aligned}
$$

where the function $\gamma: \mathbb{N}^{2} \rightarrow(0, \infty)$ satisfies

$$
n_{2} \gamma\left(n_{1}, n_{2}+n_{3}\right) \max \left\{1,\left[\frac{d\left(n_{2}+n_{3}\right)}{d\left(n_{2}\right)}\right]^{2 d-1}\right\} \leq\left(n_{2}+n_{3}\right) \gamma\left(n_{1}, n_{2}\right),
$$

The proof of Lemma 3.5 is identical to that of Lemma 3.3. The proof of Lemma 3.6 is very similar to the proof of Lemma 3.3 and is omitted. 
We now bound the three terms. Of the first two, we treat only $A_{1}$, the other being bounded by an identical argument. By multiplying out the brackets appearing in the definition of $A_{1}$ and using the facts that

and

$$
u^{\epsilon}\left(x_{i}-x_{j}+z\right)=\epsilon^{2-d} u\left(\frac{x_{i}-x_{j}+z}{\epsilon}\right)
$$

$$
\nabla_{x_{i}} u^{\epsilon}\left(x_{i}-x_{j}+z\right)=\epsilon^{1-d} \nabla u\left(\frac{x_{i}-x_{j}+z}{\epsilon}\right),
$$

we obtain that $A_{1}$ is bounded above by

$$
\begin{array}{r}
C \epsilon^{2(d-3)} \sum_{i, j, k \in I_{q}}|\nabla u|\left(\frac{x_{i}-x_{j}+z}{\epsilon}\right)|\nabla u|\left(\frac{x_{i}-x_{k}+z}{\epsilon}\right) J^{2}\left(x_{i}, m_{i}\right) \\
\left|\bar{J}\left(x_{j}, m_{j}\right)\right|\left|\bar{J}\left(x_{k}, m_{k}\right)\right| \mathbb{1}\left\{m_{i}=M_{1}, m_{j}=m_{k}=M_{2}\right\} \\
+C \epsilon^{2(d-2)} \sum_{i, j, k \in I_{q}} u\left(\frac{x_{i}-x_{j}+z}{\epsilon}\right) u\left(\frac{x_{i}-x_{k}+z}{\epsilon}\right)\left|\nabla J\left(x_{i}, m_{i}\right)\right|^{2} \\
\left|\bar{J}\left(x_{j}, m_{j}\right)\right|\left|\bar{J}\left(x_{k}, m_{k}\right)\right| \mathbb{1}\left\{m_{i}=M_{1}, m_{j}=m_{k}=M_{2}\right\} .
\end{array}
$$

Let us assume that $z=0$ because this will not affect out arguments. We are required to bound the quantity appearing in the statement of Lemma 3.6, for each of the following cases:

$$
\left(t, v, a_{1}, a_{2}, a_{3}\right) \in\left\{\left(|\nabla u|,|\nabla u|, J^{2},|\bar{J}|,|\bar{J}|\right),\left(u, u,|\nabla J|^{2},|\bar{J}|,|\bar{J}|\right)\right\} .
$$

Recall that each of the test functions $J, \bar{J}$, and their gradients, is assumed to be uniformly bounded with compact support. To each of the two cases, Lemma 3.6 applies. For either of them, the righthand-side of the inequality in Lemma 3.6 may be written as a finite sum of the expectations appearing there, with the sum being taken over triples of given masses $n_{1}, n_{2}$ and $n_{3}$. Such an expectation is bounded above by

$$
\begin{aligned}
C \epsilon^{3(2-d)} & \sum_{n_{2}} \iint_{K^{3}}\left(\frac{\left|x_{1}-z^{\prime}\right|^{2}}{d\left(n_{1}\right)}+\frac{\left|x_{2}-y\right|^{2}}{d\left(n_{2}\right)}+\frac{\left|x_{3}-y^{\prime}\right|^{2}}{d\left(n_{3}\right)}\right)^{\frac{-3 d+2}{2}} \\
& t\left(\frac{z^{\prime}-y}{\epsilon}\right) v\left(\frac{z^{\prime}-y^{\prime}}{\epsilon}\right) h_{n_{1}}\left(x_{1}\right) h_{n_{2}}\left(x_{2}\right) h_{n_{3}}\left(x_{3}\right) d z^{\prime} d y d y^{\prime} d x_{1} d x_{2} d x_{3},
\end{aligned}
$$

where $K=\{x:|x| \leq \ell\} \subseteq \mathbb{R}^{d}$ is chosen to contain the support of $J$ and $\bar{J}$. We bound (3.57) for the first of the two cases in (3.56), the other three being similar. In this case, $t=v=|\nabla u|$, the expression (3.57) equals

$$
C \epsilon^{3(2-d)}\left[\int_{K^{3}}|\nabla u|\left(\frac{z^{\prime}-y}{\epsilon}\right)|\nabla u|\left(\frac{z^{\prime}-y^{\prime}}{\epsilon}\right) G\left(z^{\prime}, y, y^{\prime}\right) d z^{\prime} d y d y^{\prime}\right],
$$

where $G\left(z^{\prime}, y, y^{\prime}\right)$ is defined to be

$$
\sum_{n_{2}} \int\left(\frac{\left|x_{1}-z^{\prime}\right|^{2}}{d\left(n_{1}\right)}+\frac{\left|x_{2}-y\right|^{2}}{d\left(n_{2}\right)}+\frac{\left|x_{3}-y^{\prime}\right|^{2}}{d\left(n_{3}\right)}\right)^{\frac{-3 d+2}{2}} h_{n_{1}}\left(x_{1}\right) h_{n_{2}}\left(x_{2}\right) h_{n_{3}}\left(x_{3}\right) d x_{1} d x_{2} d x_{3} .
$$

As in the proof of the fifth part of Lemma 3.5 we can show that $G \in L_{l o c}^{\infty}$. To bound the expression

$$
\int_{K^{3}}|\nabla u|\left(\frac{z^{\prime}-y}{\epsilon}\right)|\nabla u|\left(\frac{z^{\prime}-y^{\prime}}{\epsilon}\right) d z^{\prime} d y d y^{\prime}
$$


note that, for $z^{\prime} \in K$,

$$
\int_{K^{2}} d y d y^{\prime}|\nabla u|\left(\frac{z^{\prime}-y}{\epsilon}\right)|\nabla u|\left(\frac{z^{\prime}-y^{\prime}}{\epsilon}\right) \leq\left[\int_{|a| \leq 2 \ell}|\nabla u|\left(\frac{a}{\epsilon}\right) d a\right]^{2} .
$$

Using $|\nabla u|(x) \leq C|x|^{1-d}$ yields a bound $C \ell^{2} \epsilon^{2(d-1)}$ for (3.58). Hence, the expression appearing in (3.57) is bounded above by $C \epsilon^{3(2-d)} \epsilon^{2(d-1)}$.

Similar reasoning applies in the second case listed in (3.56). The bound on the term in (3.57) in this case is a constant multiple of $\epsilon^{3(2-d)} \epsilon^{2(d-2)}$. Applying Lemma 3.6 to the expression in (3.55), we find that

$$
\mathbb{E}_{N} \int_{0}^{T} A_{1}(q(t)) d t \leq C \epsilon^{2(d-3)} \epsilon^{3(2-d)} \epsilon^{2(d-1)}+C \epsilon^{2(d-2)} \epsilon^{3(2-d)} \epsilon^{2(d-2)} .
$$

These bounds correspond to the two cases appearing in (3.56). Thus,

$$
\mathbb{E}_{N} \int_{0}^{T} A_{1}(q(t)) d t \leq C \epsilon^{d-2}
$$

We must treat the third term, $A_{3}$. An application of the inequality

$$
\left(a_{1}+\ldots+a_{n}\right)^{2} \leq n\left(a_{1}^{2}+\ldots+a_{n}^{2}\right)
$$

to the bound on $A_{3}$ provided in (3.50) implies that

$$
A_{3}(q) \leq \frac{9}{2} \epsilon^{4(d-2)} \sum_{i, j \in I_{q}} \alpha\left(m_{i}, m_{j}\right) \epsilon^{-2} V\left(\frac{x_{i}-x_{j}}{\epsilon}\right)\left[\sum_{n=1}^{8}\left(\sum_{k \in I_{q}} Y_{n}\right)^{2}+Y_{9}^{2}\right],
$$

where $Y_{1}$ is given by

$$
\frac{m_{i}}{m_{i}+m_{j}} u^{\epsilon}\left(x_{i}-x_{k}+z\right) J\left(x_{i}, m_{i}+m_{j}\right) \bar{J}\left(x_{k}, m_{k}\right),
$$

and where $\left\{Y_{i}: i \in\{2, \ldots, 8\}\right\}$ denote the other seven expressions in (3.50) that appear in a sum over $k \in I_{q}$, while $Y_{9}$ denotes the last term in (3.50) that does not appear in this sum. There are nine cases to consider. The first eight are practically identical, and we treat only the fifth. Note that

$$
\begin{aligned}
& \epsilon^{4(d-2)} \sum_{i, j \in I_{q}} \alpha\left(m_{i}, m_{j}\right) V_{\varepsilon}\left(x_{i}-x_{j}\right)\left(\sum_{k \in I_{q}} Y_{5}\right)^{2} \\
=C \epsilon^{2 d-4} \sum_{i, j \in I_{q}} \alpha\left(m_{i}, m_{j}\right) V_{\varepsilon}\left(x_{i}-x_{j}\right) & \\
& {\left[\sum_{k, l \in I_{q}} u\left(\frac{x_{i}-x_{k}+z}{\epsilon}\right) u\left(\frac{x_{i}-x_{l}+z}{\epsilon}\right) J^{2}\left(x_{i}, m_{i}\right) \bar{J}\left(x_{k}, m_{k}\right) \bar{J}\left(x_{l}, m_{l}\right)\right] . }
\end{aligned}
$$

In the sum with indices involving $k, l \in I_{q}$, we permit the possibility that these two may be equal, though they must be distinct from each of $i$ and $j$ (which of course must themselves be distinct by the overall convention).

Note that the expression (3.60) appears in the statement of Lemma 3.7 provided that the choice

$$
\left(v, w, a_{1}, a_{2}, a_{3}\right)=\left(u, u, J^{2},|\bar{J}|,|\bar{J}|\right)
$$


is made. Again we set $z=0$ because this does not affect the estimates. Given that the support of the functions $a_{1}, a_{2}, a_{3}: \mathbb{R}^{4 d} \rightarrow[0, \infty)$ are bounded, we must bound

$$
\begin{aligned}
\sum_{n_{2}} & \iint_{L^{4}}\left(\frac{\left|x_{1}-\hat{z}\right|^{2}}{d\left(n_{1}\right)}+\frac{\left|x_{2}-z^{\prime}\right|^{2}}{d\left(n_{2}\right)}+\frac{\left|x_{3}-y\right|^{2}}{d\left(n_{3}\right)}+\frac{\left|x_{4}-y^{\prime}\right|^{2}}{d\left(n_{4}\right)}\right)^{-2 d+1} V\left(\frac{\hat{z}-z^{\prime}}{\epsilon}\right) \\
& u\left(\frac{\hat{z}-y}{\epsilon}\right) u\left(\frac{z^{\prime}-y^{\prime}}{\epsilon}\right) h_{n_{1}}\left(x_{1}\right) h_{n_{2}}\left(x_{2}\right) h_{n_{3}}\left(x_{3}\right) h_{n_{4}}\left(x_{4}\right) d \hat{z} d z^{\prime} d y d y^{\prime} d x_{1} d x_{2} d x_{3} d x_{4},
\end{aligned}
$$

for a compact set $L$. This expression is bounded above by

$$
\begin{gathered}
\int_{L^{4}} V\left(\frac{\hat{z}-z^{\prime}}{\epsilon}\right) u\left(\frac{\hat{z}-y}{\epsilon}\right) u\left(\frac{z^{\prime}-y^{\prime}}{\epsilon}\right) d \hat{z} d z^{\prime} d y d y^{\prime} \\
\sum_{n_{2}} \int_{K^{4}}\left(\frac{\left|x_{1}-\hat{z}\right|^{2}}{d\left(n_{1}\right)}+\frac{\left|x_{2}-z^{\prime}\right|^{2}}{d\left(n_{2}\right)}+\frac{\left|x_{3}-y\right|^{2}}{d\left(n_{3}\right)}+\frac{\left|x_{4}-y^{\prime}\right|^{2}}{d\left(n_{4}\right)}\right)^{-2 d+1} \\
h_{n_{1}}\left(x_{1}\right) h_{n_{2}}\left(x_{2}\right) h_{n_{3}}\left(x_{3}\right) h_{n_{4}}\left(x_{4}\right) d x_{1} d x_{2} d x_{3} d x_{4},
\end{gathered}
$$

which is less than

$$
C \int_{L^{4}} V\left(\frac{\hat{z}-z^{\prime}}{\epsilon}\right) u\left(\frac{\hat{z}-y}{\epsilon}\right) u\left(\frac{z^{\prime}-y^{\prime}}{\epsilon}\right) d \hat{z} d z^{\prime} d y d y^{\prime} .
$$

The proof of this follows the proof of the fifth part of Lemma 3.5; we use the elementary inequality $a b c d \leq\left(a^{2}+b^{2}+c^{2}+d^{2}\right)^{2}$ and the fact that the function

$$
\hat{k}(x)=\sum_{n} d(n)^{d / 2-1 / 4} \int h_{n}(y)|x-y|^{1 / 2-d} d y
$$

is locally bounded. Since $\int_{L} u\left(\frac{\hat{z}-y}{\epsilon}\right) d y$ is bounded above by $C \epsilon^{d-2}$, we have that

$$
\int_{L^{4}} V\left(\frac{\hat{z}-z^{\prime}}{\epsilon}\right) u\left(\frac{\hat{z}-y}{\epsilon}\right) u\left(\frac{z^{\prime}-y^{\prime}}{\epsilon}\right) d \hat{z} d z^{\prime} d y d y^{\prime} \leq C \epsilon^{2(d-2)} \int_{L^{2}} V\left(\frac{z-z^{\prime}}{\epsilon}\right) d \hat{z} d z^{\prime} .
$$

This is at most $C \varepsilon^{3 d-4}$. Applying Lemma 3.7, we find that the contribution to

$$
\mathbb{E}_{N} \int_{0}^{T} A_{3}(q(t)) d t
$$

arising from the fifth term in (3.59) is at most $C \epsilon^{2 d-4} \epsilon^{4(2-d)} \epsilon^{3 d-4}=\epsilon^{d}$.

We now treat the ninth term, as they are classified in (3.59). It takes the form

$$
\epsilon^{4 d-8} \sum_{i, j \in I_{q}} \alpha\left(m_{i}, m_{j}\right) V_{\varepsilon}\left(x_{i}-x_{j}\right) u^{\epsilon}\left(x_{i}-x_{j}+z\right)^{2} J\left(x_{i}, m_{i}\right)^{2} \bar{J}\left(x_{j}, m_{j}\right)^{2} .
$$

This is bounded above by

$$
C \epsilon^{2 d-4} \sum_{i, j \in I_{q}} \alpha\left(m_{i}, m_{j}\right) V_{\varepsilon}\left(x_{i}-x_{j}\right)
$$

because $u^{\varepsilon} \leq C \varepsilon^{2-d}$ by (3.20). The expected value of the integral on the interval of time $[0, T]$ of this last expression is bounded above by

$$
C \varepsilon^{2 d-4} \mathbb{E}_{N} \int_{0}^{T} d t \sum_{i, j \in I_{q}} \alpha\left(m_{i}, m_{j}\right) V_{\varepsilon}\left(x_{i}-x_{j}\right) \leq C \varepsilon^{d-2},
$$

where we used Lemma 3.1 for the last inequality. This completes the proof of (3.11). 
3.5. Using the estimates. We now use the estimates derived in the preceding sections to prove the Stosszahlansatz. The precise form of the Stosszahlansatz that we derive is now stated.

Proposition 2. Recall from (2.3) that

$$
Q(0)=\epsilon^{d-2} \sum_{(i, j) \in I_{q}} \alpha\left(m_{i}, m_{j}\right) V_{\epsilon}\left(x_{i}-x_{j}\right) J\left(x_{i}, m_{i}\right) \bar{J}\left(x_{j}, m_{j}\right)
$$

where, as before, we simply write $J\left(x_{i}, m_{i}\right)$ and $\bar{J}\left(x_{i}, m_{i}\right)$ for $J\left(x_{i}, m_{i}, t\right)$ and $\bar{J}\left(x_{i}, m_{i}, t\right)$. Let $\eta: \mathbb{R}^{d} \rightarrow[0, \infty)$ denote a smooth function of compact support for which $\int_{\mathbb{R}^{d}} \eta(x) d x=1$. We have that

$$
\begin{aligned}
& \int_{0}^{T} Q(0)(t) d t \\
= & \int_{0}^{T} d t \int_{\mathbb{R}^{d}} d \omega \beta\left(M_{1}, M_{2}\right) J\left(\omega, M_{1}\right) \bar{J}\left(\omega, M_{2}\right)\left[\epsilon^{d-2} \sum_{i \in I_{q}: m_{i}=M_{1}} \delta^{-d} \eta\left(\frac{x_{i}-\omega}{\delta}\right)\right] \\
& {\left[\epsilon^{d-2} \sum_{j \in I_{q} ; m_{j}=M_{2}} \delta^{-d} \eta\left(\frac{x_{j}-\omega}{\delta}\right)\right]+\operatorname{Err}(\epsilon, \delta), }
\end{aligned}
$$

where the constants $\beta: \mathbb{N}^{2} \rightarrow[0, \infty)$ were defined in (1.8), and where the function Err satisfies

$$
\lim _{\delta \downarrow 0} \limsup _{\epsilon \downarrow 0} \mathbb{E}_{N}|\operatorname{Err}(\epsilon, \delta)|=0
$$

Proof. Note firstly that the inequalities (3.9), (3.10) and (3.11) imply that, for large $T$,

$$
\lim _{|z| \rightarrow 0} \limsup _{\epsilon \downarrow 0} \mathbb{E}_{N}\left|\int_{0}^{T} H_{11}(t) d t+\int_{0}^{T} H_{13}(t) d t\right|=0 .
$$

That is,

$$
\begin{aligned}
& \lim _{|z| \rightarrow 0} \limsup _{\epsilon \downarrow 0} \epsilon^{2(d-2)} \mathbb{E}_{N} \mid \int_{0}^{T} d t \sum_{i, j \in I_{q(t)}} \alpha\left(m_{i}, m_{j}\right) J\left(x_{i}, m_{i}\right) \bar{J}\left(x_{j}, m_{j}\right) \\
& \left.+\int_{0}^{T} d t \sum_{i, j \in I_{q(t)}} \alpha\left(V^{\epsilon}\left(x_{i}-x_{j}+z\right)-m_{j}\right) J\left(x_{i}, m_{i}\right) \bar{J}\left(x_{j}, m_{j}\right)\right] \\
& V_{\epsilon}\left(x_{i}-x_{j}+z\right) u^{\epsilon}\left(x_{i}-x_{j}+z\right) \mid=0 .
\end{aligned}
$$


This implies that

$$
\begin{aligned}
& \epsilon^{2(d-2)} \int_{0}^{T} d t \sum_{i, j \in I_{q(t)}} \alpha\left(m_{i}, m_{j}\right) V^{\epsilon}\left(x_{i}-x_{j}\right) J\left(x_{i}, m_{i}\right) \bar{J}\left(x_{j}, m_{j}\right) \\
= & \epsilon^{2(d-2)} \int_{0}^{T} d t \sum_{i, j \in I_{q(t)}} \alpha\left(m_{i}, m_{j}\right) V^{\epsilon}\left(x_{i}-x_{j}+z\right) J\left(x_{i}, m_{i}\right) \bar{J}\left(x_{j}, m_{j}\right) \\
& {\left[1+\epsilon^{d-2} u^{\epsilon}\left(x_{i}-x_{j}+z\right)\right]+\operatorname{err}(\epsilon, z) } \\
= & \epsilon^{2(d-2)} \int_{0}^{T} d t \sum_{i, j \in I_{q(t)}} \alpha\left(m_{i}, m_{j}\right) \epsilon^{-d} U_{m_{i}, m_{j}}\left(\frac{x_{i}-x_{j}+z}{\epsilon}\right) J\left(x_{i}, m_{i}\right) \bar{J}\left(x_{j}, m_{j}\right)+\operatorname{err}(\epsilon, z),
\end{aligned}
$$

where

$$
U_{m_{1}, m_{2}}(x)=V(x)\left[1+u_{m_{1}, m_{2}}(x)\right]
$$

and where $\operatorname{err}$ satisfies

$$
\lim _{|z| \rightarrow 0} \limsup _{\epsilon \downarrow 0} \mathbb{E}_{N}|\operatorname{err}(\epsilon, z)|=0 .
$$

Writing

$$
\bar{Q}(z)=\epsilon^{d-2} \sum_{i, j \in I_{q}} \alpha\left(m_{i}, m_{j}\right) \epsilon^{-2} U_{m_{i}, m_{j}}\left(\frac{x_{i}-x_{j}+z}{\epsilon}\right) J\left(x_{i}, m_{i}\right) \bar{J}\left(x_{j}, m_{j}\right),
$$

it follows from (3.66) that

$$
\int_{0}^{T} Q(0)(t) d t=\int_{0}^{T} \bar{Q}(z)(t) d t+\operatorname{err}(\epsilon, z) .
$$

Note that

$$
\begin{aligned}
&\left|\bar{Q}\left(z_{2}-z_{1}\right)-\epsilon^{d-2} \sum_{i, j \in I_{q}} \alpha\left(m_{i}, m_{j}\right) \epsilon^{-2} U_{m_{i}, m_{j}}\left(\frac{x_{i}-x_{j}+z_{2}-z_{1}}{\epsilon}\right) J\left(x_{i}-z_{1}, m_{i}\right) \bar{J}\left(x_{j}-z_{2}, m_{j}\right)\right| \\
& \leq \epsilon^{d-2} \sum_{i, j \in I_{q}} \alpha\left(m_{i}, m_{j}\right) \epsilon^{-2} U_{m_{i}, m_{j}}\left(\frac{x_{i}-x_{j}+z_{2}-z_{1}}{\epsilon}\right) \\
&(3.71) \quad\left|\left(J\left(x_{i}-z_{1}, m_{i}\right)-J\left(x_{i}, m_{i}\right)\right) \bar{J}\left(x_{j}-z_{2}, m_{j}\right)+J\left(x_{i}, m_{i}\right)\left(J\left(x_{j}-z_{2}, m_{j}\right)-J\left(x_{j}, m_{j}\right)\right)\right| \\
& \leq \quad \epsilon^{d-2} \sum_{i, j \in I_{q}} \alpha\left(m_{i}, m_{j}\right) \epsilon^{-2} U_{m_{i}, m_{j}}\left(\frac{x_{i}-x_{j}+z_{2}-z_{1}}{\epsilon}\right)\left(\left|z_{1}\right|+\left|z_{2}\right|\right) K\left(x_{i}, m_{i}\right) \bar{K}\left(x_{j}, m_{j}\right),
\end{aligned}
$$

for small $z_{1}, z_{2}$, where $K, \bar{K}: \mathbb{N} \times \mathbb{R}^{d} \times[0, \infty) \rightarrow[0, \infty)$ are two smooth compactly supported test functions satisfying

$$
K, \bar{K} \leq 2(\|\nabla J\|+\|J\|) \mathbb{1}\left\{x \in \mathbb{R}^{d}: d(x, \operatorname{supp} J \cup \operatorname{supp} \bar{J}) \leq 1\right\} .
$$

Note that the final term in (3.71) is equal to $\left(\left|z_{1}\right|+\left|z_{2}\right|\right) \bar{Q}_{K}\left(z_{2}-z_{1}\right)$, where $Q_{K}$ and $\bar{Q}_{K}$ are defined by (3.63) and (3.69) with $K$ and $\bar{K}$ replacing $J$ and $\bar{J}$. By (3.70) applied with $Q_{K}(0)$ and $\bar{Q}_{K}(z)$ 
in place of $Q(0)$ and $\bar{Q}(z)$, and Lemma 3.1, we find that

$$
\epsilon^{d-2} \mathbb{E}_{N}\left|\int_{0}^{T} d t \sum_{i, j \in I_{q}} \alpha\left(m_{i}, m_{j}\right) \epsilon^{-2} U_{m_{i}, m_{j}}\left(\frac{x_{i}-x_{j}+z_{2}-z_{1}}{\epsilon}\right) K\left(x_{i}, m_{i}\right) \bar{K}\left(x_{j}, m_{j}\right)\right| \leq Z\|K\|\|\bar{K}\| .
$$

By (3.71) and (3.72),

$$
\begin{array}{r}
\bar{Q}\left(z_{2}-z_{1}\right)=\epsilon^{d-2} \sum_{i, j \in I_{q}} \alpha\left(m_{i}, m_{j}\right) \epsilon^{-2} U_{m_{i}, m_{j}}\left(\frac{x_{i}-x_{j}+z_{2}-z_{1}}{\epsilon}\right) \\
J\left(x_{i}-z_{1}, m_{i}\right) \bar{J}\left(x_{j}-z_{2}, m_{j}\right)+\operatorname{err}\left(\epsilon, z_{1}, z_{2}\right),
\end{array}
$$

where we have that $\mathbb{E}_{N}\left|\operatorname{err}\left(\epsilon, z_{1}, z_{2}\right)\right| \leq Z|| K|||| \bar{K}||\left(\left|z_{1}\right|+\left|z_{2}\right|\right)$. By (‥70) and (3.73),

$$
\begin{aligned}
& \int_{0}^{T} Q(0)(t) d t \\
= & \epsilon^{d-4} \int_{0}^{T} d t \sum_{i, j \in I_{q(t)}} J\left(x_{i}-z_{1}, m_{i}\right) \bar{J}\left(x_{j}-z_{2}, m_{j}\right) \alpha\left(m_{i}, m_{j}\right) \\
& \int_{\mathbb{R}^{d}} \int_{\mathbb{R}^{d}} U_{m_{i}, m_{j}}\left(\frac{\left(x_{i}-z_{1}\right)-\left(x_{j}-z_{2}\right)}{\epsilon}\right) \delta^{-d} \eta\left(\frac{z_{1}}{\delta}\right) \delta^{-d} \eta\left(\frac{z_{2}}{\delta}\right) d z_{1} d z_{2}+\operatorname{err}(\epsilon, \delta) \\
= & \epsilon^{d-4} \int_{0}^{T} d t \int_{\mathbb{R}^{2 d}} d \omega_{1} d \omega_{2} \sum_{i, j \in I_{q(t)}} U_{m_{i}, m_{j}}\left(\frac{\omega_{1}-\omega_{2}}{\epsilon}\right) \alpha\left(m_{i}, m_{j}\right) J\left(\omega_{1}, m_{i}\right) \bar{J}\left(\omega_{2}, m_{j}\right) \\
= & \epsilon^{-d} \int_{0}^{T} d t \int_{\mathbb{R}^{2 d}} d \omega_{1} d \omega_{2} U_{M_{1}, M_{2}}\left(\frac{\omega_{1}-\omega_{2}}{\epsilon}\right) \alpha\left(M_{1}, M_{2}\right) J\left(\omega_{1}, M_{1}\right) \bar{J}\left(\omega_{2}, M_{2}\right) \\
& {\left[\epsilon^{d-2} \sum_{i \in I_{q}: m_{i}=M_{1}} \delta^{-d} \eta\left(\frac{x_{i}-\omega_{1}}{\delta}\right)\right]\left[\epsilon_{j}^{d-2} \sum_{j \in I_{q} ; m_{j}=M_{2}} \delta^{-d} \eta\left(\frac{x_{j}-\omega_{2}}{\delta}\right)\right]+\operatorname{err}(\epsilon, \delta) }
\end{aligned}
$$

where the function err satisfies

$$
\lim _{\delta \downarrow 0} \limsup _{\epsilon_{\downarrow} 0} \mathbb{E}_{N}|\operatorname{err}(\epsilon, \delta)|=0,
$$

and where in the last equality, we made use of the fact that the test functions $J$ and $\bar{J}$ take non-zero values only on particles of a given mass, respectively $M_{1}$ and $M_{2}$. Recalling (1.8) and (3.67). and making use of the continuity of these test functions, we obtain the statement in the proposition with an error term of the form $\operatorname{Err}(\epsilon, \delta)=\operatorname{err}(\epsilon, \delta)+\operatorname{err}^{\prime}(\epsilon, \delta)$, where the latter error $\operatorname{err}^{\prime}=O\left(\epsilon \delta^{-2 d-1}\right)$. This completes the proof of Proposition 2 .

\section{UNIFORM BOUNDEDNESS OF THE MACROSCOPIC DENSITIES}

Before discussing the main goals of this section, let us review what has been achieved so far and what remains to be done in order to complete the proof of the main Theorem 1.1. Our Stosszahlansatz, stated in Proposition 2, allows us to replace the microscopic coagulation term with an expression that involves $\left(f_{n}^{\varepsilon} * \eta^{\delta}\right)\left(f_{m}^{\varepsilon} * \eta^{\delta}\right)$ with an error that goes to zero as $\varepsilon$ and $\delta$ go to zero 
in this order. Here $f_{n}^{\varepsilon}$ is the microscopic density of particles of size $n$ and $\eta^{\delta}$ is an approximation to the identity. After passing to the limit $\varepsilon \rightarrow 0$, we will have expressions of the form

$$
\int\left(f_{n} * \eta^{\delta}\right)\left(f_{m} * \eta^{\delta}\right) J d x+\operatorname{Err}(\delta)
$$

where $f_{n}$ now represents the macroscopic density, $J$ is a bounded continuous test function, and $\operatorname{Err}(\delta)$ represents an error term that goes to 0 as $\delta \rightarrow 0$. Recall that by the Lebesgue's theorem, the sequence $\left\{f_{n} * \eta^{\delta}\right\}$ converges to $f_{n}$ almost everywhere. To derive the PDE (1.6), we need to show that in (4.1) we can pass to the limit $\delta \rightarrow 0$ and replace $f_{n} * \eta^{\delta}$ with $f_{n}$. This can be achieved if we can establish the uniform integrability of the family

$$
\left\{\left(f_{n} * \eta^{\delta}\right)\left(f_{m} * \eta^{\delta}\right): \delta>0\right\}
$$

To prepare for this, let us consider the transformation

$$
q(\cdot) \mapsto g_{n}(d x, d t)=\varepsilon^{d-2} \sum_{i \in I_{q(t)}} \delta_{x_{i}(t)}(d x) \mathbb{1}\left(m_{i}(t)=n\right) d t \in \mathcal{M},
$$

where $\mathcal{M}$ denotes the space of measures $\mu_{n}(d x, d t)$ on the set $\mathbb{R}^{d} \times[0, T]$ such that $\mu_{n} \geq 0$ for every $n$, with $\mu_{n}\left(\mathbb{R}^{d} \times[0, T]\right) \leq T Z$. We use this transformation to define the probability measure $\mathcal{P}_{N}$ on $\mathcal{M}^{\mathbb{N}}$ as the pull-back of $\mathbb{P}_{N}$. We equip $\mathcal{M}$ with the topology of vague convergence and $\mathcal{M}^{\mathbb{N}}$ with the product topology so that $\mathcal{M}^{\mathbb{N}}$ is a compact metrizable space. We now outline our strategy for the proof of the uniform integrability of (4.2). Let $\mathcal{P}$ denote a limit point of the sequence $\mathcal{P}_{N}$.

(1) We show that $\mathcal{P}$ is concentrated on the space of measure sequences $\mu=\left(\mu_{n}: n \in \mathbb{N}\right)$ such that $\mu_{1}(d x, d t)=f_{1}(x, t) d x d t$ with $f_{1}(x, t) \leq k_{1}$ almost everywhere, where $k_{1}=\max _{x} h_{1}(x)$.

(2) Assume that for every $l<n$, there exists a constant $k_{l}$ such that the measure $\mathcal{P}$ is concentrated on the space of $\mu$ with $\mu_{l}(d x, d t)=f_{l}(x, t) d x d t$ for a function $f_{l}$ satisfying $f_{l}(x, t) \leq k_{l}$ almost everywhere for every $l<n$. We then deduce that $\mu_{n}(d x, d t)=f_{n}(x, t) d x d t$ with a function $f_{n}$ weakly satisfying

$$
\frac{\partial f_{n}}{\partial t}(x, t)=d(n) \Delta f_{n}(x, t)+Q_{1}^{n}(f)(x, t)-Y_{n},
$$

where $Q_{1}^{n}$ is defined as in (1.2) and $Y_{n} \geq 0$. In other words, we can determine the form of the coagulation gain term but all we can say about the loss term is that $Y \geq 0$.

(3) Under the assumptions of the previous step, we show that the function $f_{n}$ satisfies

$$
\int_{0}^{T} \int f_{n}(x, t)^{p} \exp (-|x|) d x d t<\infty
$$

for every $p>2, \mathcal{P}$-almost surely.

(4) Under these same assumptions, we show that with probability 1 with respect to $\mathcal{P}$, the density $f_{n}$ satisfies

$$
\frac{\partial f_{n}}{\partial t}(x, t)=d(n) \Delta f_{n}(x, t)+Q_{1}^{n}(f)(x, t)-\hat{Q}_{2}^{n}(f)(x, t),
$$

where $Q_{1}^{n}$ is defined as in (1.2) and

$$
\hat{Q}_{2}^{n}(f)=f_{n}(x, t) \sum_{m=1}^{n} \beta(m, n) f_{m}(x, t)+X_{n} .
$$


with $X_{n} \geq 0$. That is, at this stage we can only determine the form of the coagulation loss term up to mass $n$ and for the rest $X_{n}$, we can only assert that $X_{n} \geq 0$.

(5) The equation (4.5) is good enough to deduce that there exists a constant $k_{n}$ such that $f_{n}(x, t) \leq k_{n}, \mathcal{P}$-almost surely.

(6) Applying an induction on $n$ we show that $\mu_{n}(d x, d t)=f_{n}(x, t) d x d t$ with $f_{n}$ solving (1.6) weakly and that $f_{n}(x, t) \leq k_{n}$ for every $n, \mathcal{P}$-almost surely.

The main objectives of this section are Steps 1, 3 and 5 of the above outline. The remaining Steps 2, 4 and 6 will be carried out in Section 5.

Firstly, we establish the claim we made in Step 1. Throughout this Section, we let $\psi:[0, \infty) \rightarrow$ $[0, \infty)$ denote a convex and increasing function with $\psi(0)=0$.

Definition 4.1. For $n \in \mathbb{N}$, write $f^{\delta}(n, x, t)=f^{\delta}(n, x ; q(t))$ where

$$
f^{\delta}(n, x ; q)=\epsilon^{d-2} \sum_{i \in I_{q}} \eta^{\delta}\left(x-x_{i}\right) \mathbb{1}\left\{n_{i}=n\right\}
$$

where $\eta: \mathbb{R}^{d} \rightarrow[0, \infty)$ is a smooth function of compact support with $\int \eta d x=1$ and $\eta^{\delta}(z)=$ $\delta^{-d} \eta(z / \delta)$.

Note that $g_{n} *_{x} \eta^{\delta}=f^{\delta}(n, x, t) d t$ where $g_{n}$ was defined in (4.3).

Lemma 4.1. Suppose that $\psi:[0, \infty) \rightarrow[0, \infty)$ is, in addition to its other properties, chosen so that $\psi^{\prime \prime}:[0, \infty) \rightarrow[0, \infty)$ exists and has compact support. Then, for any $T, s \in[0, \infty)$ satisfying $T \geq s$,

$$
\mathbb{E}_{N} \int_{\mathbb{R}^{d}} \psi\left(f^{\delta}(1, x, T)\right) d x \leq \mathbb{E}_{N} \int_{\mathbb{R}^{d}} \psi\left(f^{\delta}(1, x, s)\right) d x+(T-s) \frac{\epsilon^{d-2}}{\delta^{d+2}} \int_{\mathbb{R}^{d}}|\nabla \eta|^{2} d x .
$$

Proof From the identity $\mathbb{E}_{N} F(q(T))=\mathbb{E}_{N} F(q(s))+\mathbb{E}_{N} \int_{s}^{T} \mathbb{L} F(q(t)) d t$, we deduce that

$$
\mathbb{E}_{N} \int_{\mathbb{R}^{d}} \psi\left(f^{\delta}(1, x, T)\right) d x=\mathbb{E}_{N} \int_{\mathbb{R}^{d}} \psi\left(f^{\delta}(1, x, s)\right) d x+A_{1}+\sum_{n=1}^{\infty} A_{1, n}
$$

where

$$
A_{1}=d(1) \mathbb{E}_{N} \int_{s}^{T} d t \int_{\mathbb{R}^{d}} \sum_{i \in I_{q(t)}} \Delta_{x_{i}} \psi\left(f^{\delta}(1, x, t)\right) \mathbb{1}\left\{m_{i}=1\right\} d x
$$

and where, for example,

$$
A_{1,1}=\alpha(1,1) \mathbb{E}_{N} \int_{s}^{T} d t \int_{\mathbb{R}^{d}} \sum_{i, j \in I_{q(t)}} V_{\epsilon}\left(x_{i}-x_{j}\right) D_{i, j}(x, t) \mathbb{1}\left\{m_{i}=m_{j}=1\right\} d x,
$$

where $D_{i, j}(x, t)$ equals

$$
\psi\left(f^{\delta}(1, x, t)-\varepsilon^{d-2} \eta^{\delta}\left(x-x_{i}\right)-\varepsilon^{d-2} \eta^{\delta}\left(x-x_{j}\right)\right)-\psi\left(f^{\delta}(1, x, t)\right) .
$$

From the monotonicity of $\psi$, we know that $D_{i, j} \leq 0$. This in turn implies that, for each $n \in \mathbb{N}$, we have that $A_{1, n} \leq 0$. 
In bounding $A_{1}$, we make use of the equality

$$
\Delta_{x_{i}} \psi\left(f^{\delta}\right)=\psi^{\prime \prime}\left(f^{\delta}\right)\left|\nabla_{x_{i}} f^{\delta}\right|^{2}+\psi^{\prime}\left(f^{\delta}\right) \Delta_{x_{i}} f^{\delta} .
$$

Since $\psi^{\prime \prime}$ is of compact support, we find that, for any given $t \in[s, T]$,

$$
\begin{aligned}
& \int_{\mathbb{R}^{d}} \mathbb{E}_{N} \sum_{i \in I_{q(t)}} \psi^{\prime \prime}\left(f^{\delta}(1, x, t)\right)\left|\nabla_{x_{i}} f^{\delta}(1, x, t)\right|^{2} \mathbb{1}\left\{m_{i}=1\right\} d x \\
\leq & C \mathbb{E}_{N} \int_{\mathbb{R}^{d}} \sum_{i \in I_{q}}\left|\nabla_{x_{i}} f^{\delta}(1, x, t)\right|^{2} \mathbb{1}\left\{m_{i}=1\right\} \\
= & C \frac{\epsilon^{2(d-2)}}{\delta^{2(d+1)}} \int_{\mathbb{R}^{d}} d x \sum_{i \in I_{q}}|\nabla \eta|^{2}\left(\frac{x-x_{i}}{\delta}\right) \mathbb{1}\left\{m_{i}=1\right\} \leq C \frac{\epsilon^{d-2}}{\delta^{d+2}} \int_{\mathbb{R}^{d}}|\nabla \eta|^{2}(x) d x,
\end{aligned}
$$

where in the last inequality, we used the fact that the total number of particles in the model is bounded above by $Z \epsilon^{2-d}$. Note also that, for $t \in[s, T]$,

$$
\begin{aligned}
& \int_{\mathbb{R}^{d}} \mathbb{E}_{N} \sum_{i \in I_{q}} \psi^{\prime}\left(f^{\delta}(1, x, t)\right) \Delta_{x_{i}} f^{\delta}(1, x, t) \mathbb{1}\left\{m_{i}=1\right\} d x \\
= & \int_{\mathbb{R}^{d}} \mathbb{E}_{N} \psi^{\prime}\left(f^{\delta}(1, x, t)\right) \Delta_{x} f^{\delta}(1, x, t) d x=-\int_{\mathbb{R}^{d}} \mathbb{E}_{N} \psi^{\prime \prime}\left(f^{\delta}(1, x, t)\right)\left|\nabla_{x} f^{\delta}(1, x, t)\right|^{2} d x \leq 0
\end{aligned}
$$

Applying the equality (4.7) to the term $A_{1}$ in (4.6) and using the bounds (4.8) and (4.9), we deduce the statement of the Lemma, each of the terms $\left\{A_{1, n}: n \in \mathbb{N}\right\}$ being non-positive.

We are now ready to establish the claim of the first step in the outline.

Lemma 4.2. Let the measure $\mathcal{P}$ be a limit point of the sequence $\left\{\mathcal{P}_{N}\right\}$. Then the measure $\mathcal{P}$ is concentrated on the space of $\mu$ such that $\mu_{1}(d x, d t)=f_{1}(x, t) d x d t$ with $f_{1}(x, t) \leq k_{1}$ almost everywhere, where $k_{1}=\max _{x} h_{1}(x)$.

Proof Fix a continuous function $\gamma:[0, \infty) \rightarrow[0, \infty)$ of compact support with $\int \gamma d t=1$. It is straightforward to show that the map

$$
\nu \mapsto F(\nu):=\int_{\mathbb{R}^{d}} \psi\left(\int \eta^{\delta}(x-y) \gamma(t) \nu(d y, d t)\right) d x,
$$

is lower semicontinuous with respect to the vague topology. By Lemma 4.1,

$$
\int \mathcal{P}_{N}(d \mu) \int_{\mathbb{R}^{d}} \psi\left(\int \eta^{\delta}(x-y) \gamma(t) \mu_{1}(d y, d t)\right) d x \leq \mathbb{E}_{N} \int \psi\left(f^{\delta}(1, x, 0)\right) d x+C \frac{\varepsilon^{d-2}}{\delta^{d+2}},
$$

where $\psi$ satisfies the conditions of Lemma 4.1 . Choose $k \in \mathbb{R}$ to satisfy $k>k_{1}=\max _{x} h_{1}(x)$. By approximation, we may choose $\psi: \mathbb{R} \rightarrow[0, \infty)$ to be $\psi(z)=(z-k)_{+}$. With this choice, we can show that the right-hand-side of (4.11) goes to 0 in the limit $N \rightarrow \infty$. The left-hand-side being non-negative, it has zero limit also. Using the lower semicontinuity of (4.10) we deduce

$$
\int \mathcal{P}(d \mu) \int_{\mathbb{R}^{d}} \psi\left(\int \eta^{\delta}(x-y) \gamma(t) \mu_{1}(d y, d t)\right) d x=0
$$


Since the function $\gamma$ is arbitrary we deduce that $\mathcal{P}(d \mu)$-almost surely, the measure $\mu_{1}$ has a density $f_{1}$ and that $f_{1}(x, t) \leq k_{1}$, for almost all $(x, t) \in \mathbb{R}^{d} \times[0, \infty)$.

We now turn to Steps 3 and 5 of the outline. The second part of Lemma 4.3 below will take care of Step 5. A microscopic version of the first part of Lemma 4.3 is out Step 5 and will be carried out in Lemma 4.4. Indeed Lemma 4.3 is a variation of a theorem of Wrzosek [10] regarding the boundedness of the solutions of (1.6). We give a detailed proof of Lemma 4.3 even though the proof is a straightforward adaptation of the arguments in [10].

Lemma 4.3. Let $J: \mathbb{R}^{d} \rightarrow(0, \infty)$ be a smooth function with $\int J d x<\infty$ such that for a constant $R>\frac{1}{2}$, we have that $|\nabla J(x)| \leq R J(x)$ for every $x$.

- Assume that $\left\{f_{j}: \mathbb{R}^{d} \times[0, \infty) \rightarrow[0, \infty), j \in\{1,2, \ldots, n\}\right\}$ denotes a collection of functions satisfying (4.4) with $Y$ nonnegative. Assume also that there exist constants $\left\{k_{1}, \ldots, k_{n-1}\right\}$ such that $\sup _{x, t} f_{n}(x, t) \leq k_{j}$ for $j<n$. Then for every $p>2$,

$$
\sup _{t} \int f_{n}(x, t)^{p} J(x) d x<\infty
$$

- In addition to the above assumptions, suppose that $f_{n}$ satisfies (4.5). Then

$$
\sup _{x, t} f_{n}(x, t)=: k_{n}<\infty
$$

Proof We first establish (4.11). Let us write $\psi_{p}(z)$ for $z^{p}$. Note that

$$
\begin{aligned}
& \frac{d}{d t} \int_{\mathbb{R}^{d}} \psi_{p}\left(f_{n}(t, x)\right) J(x) d x \\
= & \int_{\mathbb{R}^{d}} \psi_{p}^{\prime}\left(f_{n}(t, x)\right)\left[d(n)\left(\Delta f_{n}\right)(t, x)\right. \\
& \left.+\frac{1}{2} \sum_{m=1}^{n-1} \hat{\alpha}(m, n-m) f_{m}(t, x) f_{n-m}(t, x)-Y\right] J(x) d x
\end{aligned}
$$

We have that

$$
\begin{aligned}
& \int_{\mathbb{R}^{d}} \psi_{p}^{\prime}\left(f_{n}(t, x)\right)\left(\Delta f_{n}\right)(t, x) J(x) d x \\
= & -\int_{\mathbb{R}^{d}} \psi_{p}^{\prime \prime}\left(f_{n}(t, x)\right)\left|\nabla f_{n}\right|^{2}(t, x) J(x) d x \\
& -\int_{\mathbb{R}^{d}} \psi_{p}^{\prime}\left(f_{n}(t, x)\right) \nabla f_{n}(t, x) \cdot \nabla J(x) d x .
\end{aligned}
$$


We find that

$$
\begin{aligned}
& \left|\int_{\mathbb{R}^{d}} \psi_{p}^{\prime}\left(f_{n}(t, x)\right) \nabla f_{n}(t, x) \cdot \nabla J(x) d x\right| \\
\leq & R \int_{\mathbb{R}^{d}} \frac{\psi_{p}^{\prime}\left(f_{n}(t, x)\right)}{\psi_{p}^{\prime \prime}\left(f_{n}(t, x)\right)}\left|\nabla f_{n}(t, x)\right| \psi_{p}^{\prime \prime}\left(f_{n}(t, x)\right) J(x) d x \\
\leq & \frac{R}{2} \int_{\mathbb{R}^{d}} R^{2} \frac{\psi_{p}^{\prime}\left(f_{n}(t, x)\right)^{2}}{\psi_{p}^{\prime \prime}\left(f_{n}(t, x)\right)} J(x) d x+\frac{R}{2} \int_{\mathbb{R}^{d}} \frac{\left|\nabla f_{n}(t, x)\right|^{2}}{R^{2}} \psi_{p}^{\prime \prime}\left(f_{n}(t, x)\right) J(x) d x \\
\leq & R^{3} \int_{\mathbb{R}^{d}} \psi_{p}\left(f_{n}(t, x)\right) J(x) d x+\frac{1}{2 R} \int_{\mathbb{R}^{d}}\left|\nabla f_{n}(t, x)\right|^{2} \psi_{p}^{\prime \prime}\left(f_{n}(t, x)\right) J(x) d x .
\end{aligned}
$$

In the first inequality, we applied the bound $|\nabla J| \leq R J(x)$. In the third, we made use of the inequality

$$
\frac{\left|\psi_{p}^{\prime}\right|^{2}}{\psi_{p}^{\prime \prime}}=\frac{p}{p-1} \psi_{p} \leq 2 \psi_{p} .
$$

From (4.13) and (4.14), we learn that

$$
\begin{aligned}
\int_{\mathbb{R}^{d}} \psi_{p}^{\prime}\left(f_{n}(t, x)\right)\left(\Delta f_{n}\right)(t, x) J(x) d x \leq & \left(\frac{1}{2 R}-1\right) \int_{\mathbb{R}^{d}} \psi_{p}^{\prime \prime}\left(f_{n}(t, x)\right)\left|\nabla f_{n}\right|^{2}(t, x) J(x) d x \\
& +R^{3} \int_{\mathbb{R}^{d}} \psi_{p}\left(f_{n}(t, x)\right) J(x) d x \\
\leq & R^{3} \int_{\mathbb{R}^{d}} \psi_{p}\left(f_{n}(t, x)\right) J(x) d x .
\end{aligned}
$$

We find that

$$
\begin{aligned}
& \frac{d}{d t} \int_{\mathbb{R}^{d}} \psi_{p}\left(f_{n}(t, x)\right) J(x) d x \\
\leq & \frac{1}{2} \sum_{m=1}^{n-1} \hat{\alpha}(m, n-m) \int_{\mathbb{R}^{d}} \psi_{p}^{\prime}\left(f_{n}(t, x)\right) f_{m}(t, x) f_{n-m}(t, x) J(x) d x \\
& +R^{3} d(n) \int_{\mathbb{R}^{d}} \psi_{p}\left(f_{n}(t, x)\right) J(x) d x \\
\leq & A \int_{\mathbb{R}^{d}} \psi_{p}^{\prime}\left(f_{n}(t, x)\right) J(x) d x \\
& +R^{3} d(n) \int_{\mathbb{R}^{d}} \psi_{p}\left(f_{n}(t, x)\right) J(x) d x,
\end{aligned}
$$

where the uniform bounds $\sup f_{i} \leq k_{i}, i \in\{1, \ldots, n-1\}$ provided by the hyptohesis, were applied in the second inequality, and where the constant $A$ was defined by

$$
A=\frac{1}{2} \sum_{m=1}^{n-1} \hat{\alpha}(m, n-m) k_{m} k_{n-m}
$$

Note that

$$
\psi_{p}^{\prime}(z) \leq p \psi_{p}(z)+p
$$


implying that

$$
\begin{aligned}
& \frac{d}{d t} \int_{\mathbb{R}^{d}} \psi_{p}\left(f_{n}(t, x)\right) J(x) d x \\
\leq & B \int_{\mathbb{R}^{d}} \psi_{p}\left(f_{n}(t, x)\right) J(x) d x+C,
\end{aligned}
$$

where

$$
B=p A+R^{3} d(n)
$$

and

This implies that

$$
C=p A \int_{\mathbb{R}^{d}} J(x) d x
$$

$$
\sup _{t \in[0, T]} \int_{\mathbb{R}^{d}} \psi_{p}\left(f_{n}(t, x)\right) J(x) d x<\infty,
$$

and concludes the first part of the Lemma.

We now turn to the second part of the Lemma. Note that

$$
\frac{\partial}{\partial t} f_{n}(x, t)=d(n) \Delta f_{n}(x, t)+\frac{1}{2} \sum_{m=1}^{n-1} \hat{\alpha}(m, n-m) f_{m}(x, t) f_{n-m}(x, t)-\hat{\alpha}(n, n) f_{n}(x, t)^{2}-\hat{X}
$$

where $\hat{X} \geq 0$. We now choose $\psi_{p}:[0, \infty) \rightarrow[0, \infty)$ according to $\psi_{p}(z)=\left[(z-1)^{+}\right]^{p}$. A repetition of the proof of (4.15) yields

$$
\begin{aligned}
\int_{\mathbb{R}^{d}} \psi_{p}^{\prime}\left(f_{n}(t, x)\right)\left(\Delta f_{n}\right)(t, x) J(x) d x \leq & \left(\frac{1}{2 R}-1\right) \int_{\mathbb{R}^{d}} \psi_{p}^{\prime \prime}\left(f_{n}(t, x)\right)\left|\nabla f_{n}\right|^{2}(t, x) J(x) d x \\
& +R^{3} \int_{\mathbb{R}^{d}} \psi_{p}\left(f_{n}(t, x)\right) J(x) d x \\
\leq & R^{3} \int_{\mathbb{R}^{d}} \psi_{p}\left(f_{n}(t, x)\right) J(x) d x .
\end{aligned}
$$

It follows that

$$
\begin{aligned}
& \frac{d}{d t} \int_{\mathbb{R}^{d}} \psi_{p}\left(f_{n}(x, t)\right) J(x) d x \\
\leq & A \int_{\mathbb{R}^{d}} \psi_{p}^{\prime}\left(f_{n}(x, t)\right) J(x) d x+d(n) R^{3} \int_{\mathbb{R}^{d}} \psi_{p}\left(f_{n}(x, t)\right) J(x) d x \\
& -\hat{\alpha}(n, n) \int_{\mathbb{R}^{d}} f_{n}(x, t)^{2} \psi_{p}^{\prime}\left(f_{n}(x, t)\right) J(x) d x .
\end{aligned}
$$

Note that

implying that

$$
\psi_{p}^{\prime}(a) \leq r \psi_{p}(a)+\frac{p^{p}}{r^{p-1}}
$$

$$
\begin{aligned}
\frac{d}{d t} \int_{\mathbb{R}^{d}} \psi_{p}\left(f_{n}(x, t)\right) J(x) d x \leq & \left(A r+d(n) R^{3}\right) \int_{\mathbb{R}^{d}} \psi_{p}\left(f_{n}(x, t)\right) J(x) d x \\
& -\hat{\alpha}(n, n) \int_{\mathbb{R}^{d}} f_{n}(x, t)^{2} \psi_{p}^{\prime}\left(f_{n}(x, t)\right) J(x) d x . \\
& +A \frac{p^{p}}{r^{p-1}} \int_{\mathbb{R}^{d}} J(x) d x .
\end{aligned}
$$


On the other hand, $a^{2} \psi^{\prime}(a) \geq p \psi(a)$. Hence, the right-hand-side of (4.19) is bounded above by

$$
\left(A r+d(n) R^{3}\right) \int_{\mathbb{R}^{d}} \psi_{p}\left(f_{n}(x, t) J(x) d x+A \frac{p^{p}}{r^{p-1}} \int_{\mathbb{R}^{d}} J(x) d x-p \hat{\alpha}(n, n) \int_{\mathbb{R}^{d}} \psi_{p}\left(f_{n}(x, t)\right) J(x) d x .\right.
$$

It suffices to establish (4.11) for sufficiently large $p$. We choose $p$ large enough so that our choice $r=A^{-1}\left(p \hat{\alpha}(n, n)-d(n) R^{3}\right)$ for $r$ is positive. Such a choice yields

$$
\frac{d}{d t} \int_{\mathbb{R}^{d}} \psi_{p}\left(f_{n}(x, t)\right) J(x) d x \leq \frac{A^{p} p^{p}}{\left(p \hat{\alpha}(n, n)-d(n) R^{3}\right)^{p-1}} \int_{\mathbb{R}^{d}} J(x) d x .
$$

From this, it follows that

$$
\int_{\mathbb{R}^{d}} \psi_{p}\left(f_{n}(x, T)\right) J(x) d x \leq \int_{\mathbb{R}^{d}} \psi_{p}\left(h_{n}(x)\right) J(x) d x+T \frac{A^{p} p}{\left(\hat{\alpha}(n, n)-d(n) R^{3}\right)^{p-1}} \int_{\mathbb{R}^{d}} J(x) d x .
$$

By taking the $p$-th root of this inequality and then taking the value of $p$ to infinity, we deduce that

$$
\sup _{x, t} f_{n}(x, t) \leq \sup _{x} h_{n}(x)+\frac{A}{\hat{\alpha}(n, n)}
$$

We now concentrate on the third step of the outline. Recall the random measure $g_{n}$ that was defined by (4.3). For our purposes we need to regard the microscopic density as a member of the smaller space $\Lambda=L^{\infty}\left([0, T] ; \mathcal{M}_{Z}\left(\mathbb{R}^{d}\right)\right) \subset \mathcal{M}$ where $\mathcal{M}_{Z}\left(\mathbb{R}^{d}\right)$ denotes the space of nonnegative measures $\gamma$ with $\gamma\left(\mathbb{R}^{d}\right) \leq Z$. More precisely, the measure $g_{n}(d x, d t) \in \Lambda$ is absolutely continuous with respect to the Lebesgue measure for almost all $x$ and if $g_{n}(d x, d t)=g_{n}(d x, t) d t$, then $g_{n}\left(\mathbb{R}^{d}, t\right) \leq Z$ for almost all $t \in[0, T]$ and every $n$. Since we have a uniform bound $Z$ on the total measure of $g(d x, t)$ for almost every $t$, onee can readily show that indeed $\Lambda$ is a closed subset of the compact metric space $\mathcal{M}$. Consider the transformation

$$
q \mapsto(g(d x, t), X(d x, d t)) \in \hat{\mathcal{M}}=\Lambda^{\mathbb{N}} \times \mathcal{N}^{\mathbb{N}},
$$

where the random measures $(g, X)=\left(g_{n}, X_{n}: n \in \mathbb{N}\right)$ are given by

$$
\begin{aligned}
& g_{n}(t, d x)= \frac{\varepsilon^{d-2}}{2} \sum_{i \in I_{q(t)}} \delta_{x_{i}(t)}(d x) \mathbb{1}\left(m_{i}(t)=n\right), \\
& X_{n}(d x, d t)= \varepsilon^{d-2} \sum_{i, j \in I_{q(t)}} \alpha\left(m_{i}(t), m_{j}(t)\right) V_{\epsilon}\left(x_{i}(t)-x_{j}(t)\right) \mathbb{1}\left(m_{i}(t)+m_{j}(t)=n\right) \\
& \frac{1}{n}\left(m_{i}(t) \delta_{x_{i}(t)}+m_{j}(t) \delta_{x_{j}(t)}\right)(d x) d t,
\end{aligned}
$$

and $\mathcal{N}$ denotes the space of nonnegative measures on $\mathbb{R}^{d} \times[0, T]$. The transformation (4.21) defines a probability measure $\hat{\mathcal{P}}_{N}$ for each $N$ on the space $\hat{\mathcal{M}}$. This measure is an augmentation of the measure $\mathcal{P}_{N}$ that is defined on the space $\mathcal{M}^{\mathbb{N}}$. We equip $\hat{\mathcal{M}}$ with the product toplogy with $\Lambda$ and $\mathcal{N}$ both equipped with the vague topology. Certainly $\Lambda^{\mathbb{N}}$ is a compact metric space but $\mathcal{N}$ is only a separable complete metric space. As an immediate consequence of Lemma 3.1, we learn that the sequence $\left\{\hat{\mathcal{P}}_{N}\right\}$ is indeed tight. More precisely, if $\mathcal{N}_{l}$ denotes the space of measures $X \in \mathcal{N}$ such that $X\left(\mathbb{R}^{d} \times[0, T]\right) \leq l$, then $\sup _{N} \mathcal{P}_{N}\left(\mathcal{N}_{l}^{c}\right) \geq 1-C / l$, for a constant $C$. 
Lemma 4.4. Let $J$ be as in Lemma 4.3. Let $\hat{\mathcal{P}}$ be a limit point of the sequence $\left\{\hat{\mathcal{P}}_{N}\right\}$ and write $\mathcal{P}$ for the $g$-marginal of $\hat{\mathcal{P}}$. Assume that there exist constants $k_{1}, k_{2}, \ldots, k_{n-1}$, such that the measure $\mathcal{P}$ is concentrated on the set of measure sequences $g=\left(g_{n}: n \in \mathbb{N}\right)$ such that $g_{j}(d x, t)=f_{j}(x, t) d x d t$ with $\sup _{x, t} f_{j}(x, t) \leq k_{j}$ for $j=1,2, \ldots, n-1$. Then $g_{n}(d x, d t)=f_{n}(x, t) d x d t$ for $\mathcal{P}$-almost surely and

$$
\int\left\{\sup _{t \in[0, T]} \int_{\mathbb{R}^{d}} f_{n}(x, t)^{p} J(x) d x\right\} \mathcal{P}(d g)<\infty .
$$

Moreover, we have that $X_{n}(d x, d t)=\gamma_{n}(x, t) d x d t$, with

$$
\sup _{(x, t)} \gamma_{n}(x, t) \leq \sum_{m=1}^{n-1} \beta(m, n-m),
$$

$\hat{\mathcal{P}}$-almost surely.

Proof The statement of the Lemma asserts a microscopic analogue of the first part of Lemma 4.3. Again we write $\psi_{p}(z)$ for $z^{p}$. Let $\ell: \mathbb{R} \rightarrow[0, \infty)$ be a smooth function of compact support with $\int \ell=1$ and set $\hat{f}^{\delta}(n, x, t)=\int_{0}^{\infty} f^{\delta}(n, x, s) \ell(t-s) d s$. A straightforward calculation yields,

$$
\begin{aligned}
\frac{d}{d t} \hat{f}^{\delta}(n, x, t) \leq & -f^{\delta}(n, x, 0) \ell(t)+\int_{0}^{\infty} d(n)\left(\sum_{i \in I_{q}} \Delta_{x_{i}} f^{\delta}(n, x, s)\right) \ell(t-s) d s \\
+ & \int_{0}^{\infty} \frac{\varepsilon^{d-2}}{2} \sum_{m=1}^{n-1} \alpha(m, n-m) \sum_{i, j \in I_{q(s)}} V_{\epsilon}\left(x_{i}(s)-x_{j}(s)\right) \\
& \left(\frac{m_{i}}{n} \eta^{\delta}\left(x-x_{i}(s)\right)+\frac{m_{j}}{n} \eta^{\delta}\left(x-x_{j}(s)\right)\right) \\
& \mathbb{1}\left\{m_{i}(s)=m, m_{j}(s)=n-m\right\} \ell(t-s) d s \\
+ & \int_{0}^{\infty} M_{s}(x) \ell^{\prime}(t-s) d s .
\end{aligned}
$$

Here we have an inequality because we only used the gain term in applying the coagualtion operator $\mathbb{A}_{C}$, and $M_{s}$ is a martingale satisfying

$$
\mathbb{E}_{N}\left[M_{s}(x)^{2}\right]=\mathbb{E}_{N} \int_{0}^{s} A_{1}(q(t), x) d t+\mathbb{E}_{N} \int_{0}^{s} A_{2}(q(t), x) d t
$$

where $A_{1}(q, x)$ and $A_{2}(q, x)$ are respectively set equal to

$$
A_{1}(q, x)=\varepsilon^{2(d-2)} \sum_{i \in I_{q}} d(n)\left|\nabla_{x} \eta^{\delta}\right|^{2}\left(x-x_{i}\right) \mathbb{1}\left(m_{i}=n\right),
$$

and

$$
\begin{gathered}
A_{2}(q, x)=\frac{\epsilon^{2(d-2)}}{2} \sum_{i, j \in I_{q}} \alpha\left(m_{i}, m_{j}\right) V_{\epsilon}\left(x_{i}-x_{j}\right) \\
{\left[\frac{m_{i}}{m_{i}+m_{j}} K\left(x-x_{i}, m_{i}+m_{j}\right)+\frac{m_{j}}{m_{i}+m_{j}} K\left(x-x_{j}, m_{i}+m_{j}\right)\right.} \\
\left.\quad-K\left(x-x_{i}, m_{i}\right)-K\left(x-x_{j}, m_{j}\right)\right]^{2},
\end{gathered}
$$


where $K(x-y, m)=\eta^{\delta}(x-y) \mathbb{1}(m=n)$. We can readily show,

$$
\int_{\mathbb{R}^{d}} A_{1}(q, x) d x \leq C \epsilon^{2(d-2)} \delta^{-2 d-2} \sum_{i \in I_{q}} d(n) \mathbb{1}\left(m_{i}=n\right) \leq C \varepsilon^{d-2} \delta^{-d-2},
$$

$$
\begin{aligned}
\mathbb{E}_{N} \int_{0}^{s} \int_{\mathbb{R}^{d}} A_{2}(q(t), x) d x d t & \leq C \epsilon^{2(d-2)} \delta^{-2 d} \mathbb{E}_{N} \int_{0}^{s} \sum_{i, j \in I_{q(t)}} \alpha\left(m_{i}(t), m_{j}(t)\right) V_{\epsilon}\left(x_{i}(t)-x_{j}(t)\right) \\
& \leq C \varepsilon^{d-2} \delta^{-2 d},
\end{aligned}
$$

where we have used Lemma 3.1 for the last inequality.

As a result,

$$
\mathbb{E}_{N} \int_{\mathbb{R}^{d}}\left[M_{s}(x)^{2}\right] d x \leq C \varepsilon^{d-2} \delta^{-2 d-2}
$$

We certainly have,

$$
\int_{\mathbb{R}^{d}} \frac{d}{d t} \psi_{p}\left(\hat{f}^{\delta}(n, x, t)\right) J(x) d x \leq B_{1}+B_{2}+B_{3}+B_{4}
$$

where

$$
\begin{aligned}
& B_{1}=-\ell(t) \int_{\mathbb{R}^{d}} \psi_{p}^{\prime}\left(\hat{f}^{\delta}(n, x, t)\right) f^{\delta}(n, x, 0) J(x) d x \\
& B_{2}=d(n) \int_{\mathbb{R}^{d}} \psi_{p}^{\prime}\left(\hat{f}^{\delta}(n, x, t)\right)\left(\sum_{i \in I_{q}} \Delta_{x_{i}} \hat{f}^{\delta}(n, x, t)\right) J(x) d x
\end{aligned}
$$

$$
\begin{aligned}
B_{3}=\epsilon^{d-2} & \int_{0}^{\infty} d s \ell(t-s) \int_{\mathbb{R}^{d}} \psi_{p}^{\prime}\left(\hat{f}^{\delta}(n, x, t)\right) \sum_{m=1}^{n-1} \alpha(m, n-m) \\
& \sum_{i, j \in I_{q(s)}} V_{\epsilon}\left(x_{i}(s)-x_{j}(s)\right) \eta^{\delta}\left(x-x_{i}(s)\right) \mathbb{1}\left\{m_{i}(s)=m, m_{j}(s)=n-m\right\} J(x) d x,
\end{aligned}
$$

and

$$
\begin{aligned}
\mathbb{E}_{N} B_{4} & \leq \mathbb{E}_{N} \int_{0}^{\infty} d s \ell^{\prime}(t-s) \int_{\mathbb{R}^{d}} \psi_{p}^{\prime}\left(\hat{f}^{\delta}(n, x, t)\right)\left|M_{s}(x)\right| J(x) d x \\
& \leq C \delta^{-p d+d} \int_{0}^{\infty} d s \ell^{\prime}(t-s)\left\{\int_{\mathbb{R}^{d}} \mathbb{E}_{N}\left[M_{s}(x)\right]^{2} J(x) d x\right\}^{1 / 2} \\
& \leq C \ell(t) \varepsilon^{d / 2-1} \delta^{-1-p d},
\end{aligned}
$$

where we used $\hat{f}^{\delta} \leq Z \delta^{-d}$ for the the second inequality.

On the other hand,

$$
B_{2}=d(n) \int_{\mathbb{R}^{d}} \psi_{p}^{\prime}\left(\hat{f}^{\delta}(n, x, t)\right) \Delta_{x} \hat{f}^{\delta}(n, x, t) J(x) d x=B_{21}+B_{22},
$$

where

$$
B_{21}=-d(n) \int_{\mathbb{R}^{d}} \psi_{p}^{\prime \prime}\left(\hat{f}^{\delta}(n, x, t)\right)\left|\nabla_{x} \hat{f}^{\delta}(n, x, t)\right|^{2} J(x) d x
$$


and

Note that

$$
B_{22}=-d(n) \int_{\mathbb{R}^{d}} \psi_{p}^{\prime}\left(\hat{f}^{\delta}(n, x, t)\right) \nabla_{x} \hat{f}^{\delta}(n, x, t) \cdot \nabla_{x} J(x) d x
$$

$$
\begin{aligned}
\left|B_{22}\right| \leq & R d(n) \int_{\mathbb{R}^{d}} \frac{\psi_{p}^{\prime}\left(\hat{f}^{\delta}(n, x, t)\right)}{\psi_{p}^{\prime \prime}\left(\hat{f}^{\delta}(n, x, t)\right)}\left|\nabla_{x} \hat{f}^{\delta}(n, x, t)\right| \psi_{p}^{\prime \prime}\left(\hat{f}^{\delta}(n, x, t)\right) J(x) d x \\
\leq & \frac{R d(n)}{2} \int_{\mathbb{R}^{d}} R^{2} \frac{\left|\psi_{p}^{\prime}\left(\hat{f}^{\delta}(n, x, t)\right)\right|^{2}}{\psi_{p}^{\prime \prime}\left(\hat{f}^{\delta}(n, x, t)\right)} J(x) d x \\
& +\frac{R d(n)}{2} \int_{\mathbb{R}^{d}} \frac{1}{R^{2}}\left|\nabla_{x}\left(\hat{f}^{\delta}(n, x, t)\right)\right|^{2} \psi_{p}^{\prime \prime}\left(\hat{f}^{\delta}(n, x, t)\right) J(x) d x
\end{aligned}
$$

It follows that

$$
\begin{aligned}
\left|B_{2}\right| & \leq\left(1-\frac{1}{2 R}\right) B_{21}+R^{3} \int_{\mathbb{R}^{d}} \psi_{p}\left(\hat{f}^{\delta}(n, x, t)\right) J(x) d x \\
& \leq R^{3} \int_{\mathbb{R}^{d}} \psi_{p}\left(\hat{f}^{\delta}(n, x, t)\right) J(x) d x,
\end{aligned}
$$

where in the second inequality, the fact that $B_{21} \leq 0$ was used.

Note that

where

$$
B_{3} \leq \int_{0}^{\infty} d s \ell(t-s) \int_{\mathbb{R}^{d}} \psi_{p}^{\prime}\left(\hat{f}^{\delta}(n, x, t)\right) X^{\delta}(x, n ; q(s)) J(x) d x
$$

$$
\begin{gathered}
X^{\delta}(x, n ; q)=\frac{\epsilon^{d-2}}{2} \sum_{m=1}^{n-1} \alpha(m, n-m) \sum_{i, j \in I_{q}} V_{\epsilon}\left(x_{i}-x_{j}\right) \mathbb{1}\left\{m_{i}=m, m_{j}=n-m\right\} \\
\left(\frac{m_{i}}{n} \eta^{\delta}\left(x-x_{i}\right)+\frac{m_{j}}{n} \eta^{\delta}\left(x-x_{j}\right)\right) .
\end{gathered}
$$

In summary, the expression

is bounded above by,

$$
\frac{d}{d t} \int_{\mathbb{R}^{d}} \psi_{p}\left(\hat{f}^{\delta}(n, x, t)\right) J(x) d x
$$

$$
\begin{aligned}
& -\ell(t) \int_{\mathbb{R}^{d}} \psi_{p}^{\prime}\left(\hat{f}^{\delta}(n, x, t)\right) f^{\delta}(n, x, 0) J(x) d x \\
& +R^{3} \int_{\mathbb{R}^{d}} \psi_{p}\left(\hat{f}^{\delta}(n, x, t)\right) J(x) d x \\
& +\int_{0}^{\infty} d s \ell(t-s) \int_{\mathbb{R}^{d}} \psi_{p}^{\prime}\left(\hat{f}^{\delta}(n, x, t)\right) X^{\delta}(x, n ; q(s)) J(x) d x+\hat{M}^{\varepsilon},
\end{aligned}
$$

with $\hat{M}^{\varepsilon}$ satisfying

$$
\mathbb{E}_{N}\left|\hat{M}^{\varepsilon}\right| \leq C \varepsilon^{d / 2-1} \delta^{-1-p d} .
$$

Before sending $\varepsilon \rightarrow 0$ in (4.33), recall the space $\mathcal{N}_{l}$ which was defined right before the Lemma and let us observe that the functional $\Gamma: \mathcal{M} \times \mathcal{N}_{l} \rightarrow[0, \infty)$, defined by

$$
\Gamma(g, X)=\int_{0}^{\infty} \iint \psi^{\prime}\left(\int_{0}^{\infty} \int \eta^{\delta}(x-y) \ell(t-\theta) g(d y, d \theta)\right) \eta^{\delta}(x-z) J(x) \ell(t-s) X(d z, d s) d x
$$


is continuous with respect to the weak topology. Indeed, if $g^{r} \rightarrow g$ and $X^{r} \rightarrow X$, then $F^{r} \rightarrow F$ and $Y^{r} \rightarrow Y$, where

$$
\begin{aligned}
F(x) & =\int_{0}^{\infty} \int \eta^{\delta}(x-y) \ell(t-\theta) g(d y, d \theta) \\
F^{r}(x) & =\int_{0}^{\infty} \int \eta^{\delta}(x-y) \ell(t-\theta) g^{r}(d y, d \theta) \\
Y(x) & =\int_{0}^{\infty} \int \eta^{\delta}(x-y) \ell(t-\theta) X(d y, d \theta) \\
Y^{r}(x) & =\int_{0}^{\infty} \int \eta^{\delta}(x-y) \ell(t-\theta) X^{r}(d y, d \theta) .
\end{aligned}
$$

Since both sequences $\left\{F^{r}\right\}$ and $\left\{Y^{r}\right\}$ are uniformly bounded, we deduce that

$$
\lim _{r \rightarrow \infty} \int \psi^{\prime}\left(F^{r}(x)\right) Y^{r}(x) J(x) d x=\int \psi^{\prime}(F(x)) Y(x) J(x) d x .
$$

We now fix $\delta$ and send $\varepsilon$ to 0 . From (4.33) we deduce that the measure $\hat{\mathcal{P}}$ is concentrated on the space of $\left(g_{n}, X_{n}\right)$ for which

$$
\frac{d}{d t} \int \psi_{p}^{\prime}\left(\hat{f}_{n}^{\delta}\right) J d x \leq-\ell(t) \int \psi_{p}\left(\hat{f}_{n}^{\delta}\right) h_{n}^{\delta} J d x+R^{3} \int \psi_{p}\left(\hat{f}_{n}^{\delta}\right) J d x+\int \psi_{p}^{\prime}\left(\hat{f}_{n}^{\delta}\right) X_{n}^{\delta} J d x,
$$

where,

$$
\hat{f}_{n}^{\delta}=g_{n} *_{x} \eta^{\delta} *_{t} \ell, \quad h_{n}^{\delta}=h_{n} *_{x} \eta^{\delta}, \quad X_{n}^{\delta}=X *_{x} \eta^{\delta} *_{t} \ell .
$$

We now claim that

$$
X_{n}^{\delta} \leq \sum_{m=1}^{n-1} \beta(m, n-m) k_{m} k_{n-m}
$$

$\hat{\mathcal{P}}$-almost surely. By the Stosszahlansatz, as it is recorded in (3.64),

$$
\begin{aligned}
& \lim _{\delta \rightarrow 0} \limsup _{N \rightarrow \infty} \int_{\mathbb{R}^{d}} \mid \int_{0}^{\infty} \int_{\mathbb{R}^{d}} K(x, t) \\
& \quad\left(2 X_{n}(d x, d t)-\sum_{m=1}^{n-1} \beta(m, n-m) g_{m} *_{x} \eta^{\delta}(x, t) g_{n-m} *_{x} \eta^{\delta}(x, t)\right) d x d t \mid d \hat{\mathcal{P}}_{N}=0
\end{aligned}
$$

for any smooth function $K$ of compact support. From this, the continuity of the integrand with respect to $(g, X)$, and our assumptions on $g_{j}$ for $j<n$ we deduce,

$$
\lim _{\delta \rightarrow 0} \int\left[\int_{0}^{\infty} \int K(x, t)\left(2 X_{n}(d x, d t)-\sum_{m=1}^{n-1} \beta(m, n-m) k_{m} k_{n-m}\right) d x d t\right]^{+} d \hat{\mathcal{P}}=0
$$

which implies that

$$
2 \int_{0}^{\infty} \int_{\mathbb{R}^{d}} K d X_{n} \leq \sum_{m=1}^{n-1} \beta(m, n-m) k_{m} k_{n-m} \int_{0}^{\infty} \int_{\mathbb{R}^{d}} K(x, t) d x d t
$$


for $\hat{\mathcal{P}}$ - almost all realizations of the random measure $X_{n}$. So, $X_{n}=\gamma_{n} d x d t$, with $\gamma_{n}$ bounded, implying (4.37) and the second part of the Lemma. Applying (4.37) in (4.36), using $\psi_{p}^{\prime}(z) \leq$ $p \psi_{p}(z)+p$ and Gronwall's inequality, we find that

$$
\sup _{t \in[0, T]} \int_{\mathbb{R}^{d}} \psi_{p}\left(\hat{f}^{\delta}(n, x, t)\right) J(x) d x \leq c,
$$

for a constant $c$ that is independent of $\ell$. From this we can readily deduce,

$$
\sup _{t \in[0, T]} \sup _{\delta>0} \int_{\mathbb{R}^{d}} \psi_{p}\left(g_{n} *_{x} \eta^{\delta}(x, t)\right) J(x) d x<\infty,
$$

as required to derive the first part of the Lemma.

\section{DERIVING the PDE}

The aim of this section is to carry out the second and fourth steps of the outline for deriving the system of PDE (1.1) described in Section 4. We then apply an induction on the number $n$ to complete the proof of Theorem 1.1. In each case, we choose a test function $\bar{K}:[0, \infty) \times \mathbb{R}^{d} \rightarrow \mathbb{R}$ and consider the functional

$$
Y(q, t)=\epsilon^{d-2} \sum_{i \in I_{q}} K\left(t, x_{i}, m_{i}\right),
$$

where $K(t, x, m)=\bar{K}(x, t) \mathbb{1}(m=n)$. Note that

$$
\begin{aligned}
Y(q(T), T)=Y(q(0), 0) & +\int_{0}^{T} \mathbb{A}_{C}(Y)(q(t), t) d t \\
& +\int_{0}^{T} \mathbb{A}_{0}(Y)(q(t), t) d t+\int_{0}^{T} Y_{t}(q(t), t) d t+M_{T},
\end{aligned}
$$

where

$$
\begin{gathered}
\mathbb{A}_{C} Y(q, t)=\frac{\epsilon^{d-2}}{2} \sum_{i, j \in I_{q}} \alpha\left(m_{i}, m_{j}\right) V_{\epsilon}\left(x_{i}-x_{j}\right) \\
\quad\left[\frac{m_{i}}{m_{i}+m_{j}} K\left(t, x_{i}, m_{i}+m_{j}\right)+\frac{m_{j}}{m_{i}+m_{j}} K\left(t, x_{j}, m_{i}+m_{j}\right)\right. \\
\left.\quad-K\left(t, x_{i}, m_{i}\right)-K\left(t, x_{j}, m_{j}\right)\right]
\end{gathered}
$$

and where

$$
\begin{aligned}
\mathbb{A}_{0} Y(q, t) & =\epsilon^{d-2} \sum_{i \in I_{q}} d\left(m_{i}\right) \Delta_{x} K\left(t, x_{i}, m_{i}\right), \\
Y_{t}(q, t) & =\epsilon^{d-2} \sum_{i \in I_{q}} K_{t}\left(t, x_{i}, m_{i}\right) .
\end{aligned}
$$

The term $M_{T}$ is a martingale satisfying

$$
\mathbb{E}_{N}\left[M_{T}^{2}\right]=\mathbb{E}_{N} \int_{0}^{T} A_{1}(q(t), t) d t+\mathbb{E}_{N} \int_{0}^{T} A_{2}(q(t), t) d t,
$$


where $A_{1}(q, t)$ and $A_{2}(q, t)$ are respectively set equal to

$$
A_{1}(q, t)=\varepsilon^{2(d-2)} \sum_{i \in I_{q}} d\left(m_{i}\right)\left|\nabla_{x_{i}} K\right|^{2}\left(t, x_{i}, m_{i}\right)
$$

and

$$
\begin{gathered}
A_{2}(q, t)=\frac{\epsilon^{2(d-2)}}{2} \sum_{i, j \in I_{q}} \alpha\left(m_{i}, m_{j}\right) V_{\epsilon}\left(x_{i}-x_{j}\right) \\
{\left[\frac{m_{i}}{m_{i}+m_{j}} K\left(t, x_{i}, m_{i}+m_{j}\right)+\frac{m_{j}}{m_{i}+m_{j}} K\left(t, x_{j}, m_{i}+m_{j}\right)\right.} \\
\left.-K\left(t, x_{i}, m_{i}\right)-K\left(t, x_{j}, m_{j}\right)\right]^{2} .
\end{gathered}
$$

We can readily show

$$
\begin{aligned}
& A_{1}(q, t) \leq C \epsilon^{2(d-2)} \sum_{i \in I_{q}} d\left(m_{i}\right) \mathbb{1}\left(m_{i}=n\right) \leq C \varepsilon^{d-2}, \\
& A_{2}(q, t) \leq C \epsilon^{2(d-2)} \sum_{i, j \in I_{q}} \alpha\left(m_{i}, m_{j}\right) V_{\epsilon}\left(x_{i}-x_{j}\right) \mathbb{1}\left(m_{i}+m_{j} \leq n\right) \leq C \varepsilon^{d-2},
\end{aligned}
$$

where we have used Lemma 3.1 for the last inequality. From these inequalities and Doob's inequality, we deduce that the martingale tends to zero uniformly, in the $\epsilon \downarrow 0$ limit.

We rewrite the terms of (5.1) in terms of the empirical measures introduced in (4.23). We have that

$$
Y_{q}(T)=\sum_{m \in \mathbb{N}} \int_{\mathbb{R}^{d}} \bar{K}(T, x) g_{n}(d x, T)
$$

and that

$$
\int_{0}^{T} \mathbb{A}_{0} Y_{q}(t) d t=\int_{0}^{T} d(n) \int_{\mathbb{R}^{d}} \Delta_{x} \bar{K}(t, x) g_{n}(d x, t) .
$$

The Stosszahlansatz enables us to rewrite the time-averaged action of the collision operator on $Y(q(t), t)$ in terms of these empirical measures. That is, by Proposition 2 ,

$$
\begin{aligned}
\int_{0}^{T} d t \mathbb{A}_{C} Y(q(t), t)= & \int_{0}^{T} d t \int_{\mathbb{R}^{d}} d x \bar{K}(t, x) \\
& {\left[\frac{1}{2} \sum_{m=1}^{n-1} \beta(m, n-m)\left(g_{m} *_{x} \eta^{\delta}\right)(x, t)\left(g_{n-m} *_{x} \eta^{\delta}\right)(x, t)\right.} \\
& \left.-\sum_{r=1}^{\infty} \beta(n, r)\left(g_{n} *_{x} \eta^{\delta}\right)(x, t)\left(g_{r} *_{x} \eta^{\delta}\right)(x, t)\right]+\operatorname{Err}(\epsilon, \delta) .
\end{aligned}
$$

To carry out the second step of the outline, note that by the assumption, the first $n-1$ marginals in the mass variable of any weak limit of the measures $\mathcal{P}_{N}$ have bounded densities $\left\{f_{m}:[0, \infty) \times \mathbb{R}^{d} \rightarrow\right.$ $[0, \infty): m \in\{1, \ldots, n-1\}\}$ with respect to Lesbesgue measure . By passing to the limit in low $\epsilon$ 
in (5.8), we find that any weak limit $\mathcal{P}$ is concentrated on the space of $g=\left(g_{m}: m \in \mathbb{N}\right)$ such that,

$$
\begin{aligned}
& \int_{\mathbb{R}^{d}} g_{n}(d x, T) \bar{K}(T, x) d x \\
= & \int_{\mathbb{R}^{d}} g_{n}(d x, 0) \bar{K}(0, x) d x+d(n) \int_{0}^{T} d t \int_{\mathbb{R}^{d}} g_{n}(d x, t) \Delta \bar{K}(t, x) d x \\
& +\int_{0}^{T} d t \int_{\mathbb{R}^{d}} d x \bar{K}(t, x)\left[\frac{1}{2} \sum_{m=1}^{n-1} \beta(m, n-m)\left(g_{m} *_{x} \eta^{\delta}\right)(x, t)\left(g_{n-m} *_{x} \eta^{\delta}\right)(x, t)-X_{n, \delta}\right] \\
& +\operatorname{Err}(\delta),
\end{aligned}
$$

where $X_{n, \delta} \geq 0$ and the $\mathcal{P}$-expectation of $\operatorname{Err}(\delta)$ goes to zero as $\delta \downarrow 0$. We know by the third step in the outline that $g_{m}(d x, t)=f_{m}(x, t) d x$ with $f_{m}$ being uniformly bounded for $m<n$. We find that

$$
\begin{aligned}
\int_{\mathbb{R}^{d}} g_{n}(d x, T) \bar{K}(T, x) d x= & \int_{\mathbb{R}^{d}} g_{n}(d x, 0) \bar{K}(0, x) d x+d(n) \int_{0}^{T} d s \int_{0}^{s} d t \int_{\mathbb{R}^{d}} g_{n}(d x, t) \Delta \bar{K}(t, x) d x \\
& +\int_{0}^{T} d t \bar{K}(t, x)\left[\frac{1}{2} \sum_{m=1}^{n-1} \beta(m, n-m) f_{m}(x, t) f_{n-m}(x, t)-X_{n}\right],
\end{aligned}
$$

where $X_{n} \geq 0$. From this we would like to conclude that the measure $g_{n}=f_{n} d x$ and that the sequence $\left(f_{m}: m \leq n\right)$ solves (4.5) in weak form. Since we do not know the absolute continuity of $g_{n}$ with respect to the Lebesgue measure yet, let us observe that if we set $\hat{f}_{n}=g_{n} *_{x} \eta^{\delta}$, then the sequence $\left(f_{n}: n<m ; \hat{f}_{n}\right)$ does satisfy an equation that is similar to (4.5). This equation is close enough to (4.5) so that we can apply Lemma 4.3(i) to deduce that

$$
\sup _{\delta} \sup _{t} \int\left(g_{n} *_{x} \eta^{\delta}\right)^{p} J d x<\infty
$$

for every $p>2$. From this we deduce that indeed $g_{n}=f_{n} d x$ with $f_{n}$ satisfying the same $L^{p}$-bounds.

To carry out the fourth step, note that at the end of the third, we know that any weak limit of the measures $\mathcal{P}_{N}$ is concentrated on those measures on $\mathbb{R}^{d}$ having densities with respect to Lebesgue measure $\left\{f_{m}:[0, \infty) \times \mathbb{R}^{d} \rightarrow[0, \infty): m \in \mathbb{N}\right\}$ with $f_{m}$ uniformly bounded for $m<n$ and and that $f_{n} \in L^{p}\left(\mathbb{R}^{d} \times[0, T]\right)$ for each $p>2$ and $T>0$. Thus, the families $\left\{\left(f_{n} * \eta^{\delta}\right)\left(f_{m} * \eta^{\delta}\right): \mathbb{R}^{d} \times[0, \infty) \rightarrow\right.$ $[0, \infty), \delta>0\}$ are uniformly integrable for $m \leq n$. When we pass to the limit in low $\epsilon$ of (5.8) in this case, we find that,

$$
\begin{aligned}
& \int_{\mathbb{R}^{d}} f_{n}(T, x) \bar{K}(T, x) d x \\
= & \int_{\mathbb{R}^{d}} f_{n}(0, x) \bar{K}(0, x) d x+d(n) \int_{0}^{T} d t \int_{\mathbb{R}^{d}} f_{n}(x, t) \Delta \bar{K}(t, x) d x \\
& +\int_{0}^{T} d t \int_{\mathbb{R}^{d}} d x \bar{K}(t, x)\left[\frac{1}{2} \sum_{m=1}^{n-1} \beta(m, n-m) f_{m}(x, t) f_{n-m}(x, t)\right. \\
& \left.-\sum_{m=1}^{n} \beta(n, m) f_{n}(x, t) f_{m}(x, t)-Y_{n}\right]+\operatorname{Err}(\delta),
\end{aligned}
$$

where $Y_{n} \geq 0$ and the $\mathcal{P}$-expectation of $\operatorname{Err}(\delta)$ goes to zero as $\delta \downarrow 0$. 
By induction on $n$ we deduce that the functions $\left\{f_{n}: n \in \mathbb{N}\right\}$ being known to be uniformly bounded, $\mathcal{P}$-almost surely. We then apply Lebesgue's theorem to deduce that, for each $n \in \mathbb{N}$, $f_{n} * \eta^{\delta} \rightarrow f$ almost surely, as $\delta \downarrow 0$. We find that the weak limit $\mathcal{P}$ of the sequence $\mathcal{P}_{N}$ is supported on those functions $f$ satisfying the system (1.1) in weak form.

\section{Potential theory}

The purpose of this section is twofold. Firstly, we show the existence of the function $u$ that was used in the proof of the Stosszahlansatz. Secondly, we establish a bound on $\beta$ and show that if $\alpha \rightarrow \infty$ then $\beta$ converges to macroscopic coagulation rate in the case of a hard sphere interaction. The limit can be expressed in terms of the Newtonian capacity of the support of the function $V$. We start with the statements of the main results of this section. Let $V: \mathbb{R}^{d} \rightarrow \mathbb{R}$ be a continuous

function of compact support with $V \geq 0$ and $\int_{\mathbb{R}^{d}} V(x) d x=1$. We also write $K_{0}$ for the topological closure of $U_{0}$ where $U_{0}=\{x: V(x) \neq 0\}$.

Theorem 6.1. For every $\beta>0$, there exists a unique function $u \in C^{2}\left(\mathbb{R}^{d}\right)$ such that $\lim _{|x| \rightarrow \infty} u(x)=0$ and

$$
\Delta u-\beta V u=\beta V .
$$

Moreover $-1 \leq u(x) \leq 0$ for every $x \in \mathbb{R}^{d}$.

We have applied this theorem in Section 3 with $\beta=\frac{\alpha(n, m)}{d(n)+d(m)}$. Also recall that $\beta(n, m)$ is defined by

$$
\beta(n, m)=\alpha(n, m) \int_{\mathbb{R}^{d}} V(x)(1+u(x)) d x .
$$

More generally, we define $F:(0, \infty) \rightarrow(0, \infty)$ by $F(\beta)=\beta \int_{\mathbb{R}^{d}} V\left(1+u^{\beta}\right) d x$ with $u=u^{\beta}$ as in Theorem 6.1. Recall that the (Newtonian) capacity of a compact set $K \subseteq \mathbb{R}^{d}$ is given by

$$
\operatorname{Cap}(K)=\inf \left\{\frac{1}{2} \int_{\mathbb{R}^{d}}|\nabla \psi|^{2} d x: \psi \in C^{1}\left(\mathbb{R}^{d}\right), \psi \geq 1 \text { on a neighborhood of } K\right\},
$$

or equivalently,

$$
\operatorname{Cap}(K)=\sup \left\{\mu(K): \operatorname{supp} \mu \subset K, c_{0} \int_{\mathbb{R}^{d}}|x-y|^{2-d} \mu(d y) \leq 1 \text { for every } x\right\},
$$

where $c_{0}=c_{0}(d)=(d-2)^{-1} \omega_{d}^{-1}$ with $\omega_{d}$ denoting the surface area of the unit sphere. We are now ready to state our second main result.

Theorem 6.2. We always have $F(\beta) \leq \operatorname{Cap}\left(K_{0}\right)$. Moreover $\lim _{\beta \rightarrow \infty} F(\beta)=\operatorname{Cap}\left(K_{0}\right)$.

\section{Proof of Theorem 6.1}

Step 1. Let $J$ be a bounded continuous function with $J>0, \int_{\mathbb{R}^{d}} J(x) d x=\infty$ and 
$\int_{|x| \geq 1} J(x)|x|^{4-2 d} d x<\infty$. Define

$$
\mathcal{H}=\left\{u: u \text { is measurable and } \int_{\mathbb{R}^{d}} u^{2}(x) J(x) d x<\infty\right\} .
$$

We then define $\mathcal{F}: \mathcal{H} \rightarrow \mathcal{H}$ by

$$
\mathcal{F}(u)(x)=c_{0} \int_{\mathbb{R}^{d}}|x-y|^{2-d} V(y) u(y) d y .
$$

Let us verify that $\mathcal{F}(u) \in \mathcal{H}$ for $u \in \mathcal{H}$. To see this, write

$$
\Gamma(x)=c_{0} \int_{\mathbb{R}^{d}}|x-y|^{2-d} V(y) d y .
$$

As in (3.29) we can readily show

$$
0 \leq \Gamma(x) \leq c_{1} \min \left(|x|^{2-d}, 1\right)
$$

for a constant $c_{1}$. Also, we may use Hölder's inequality to assert

$$
\begin{aligned}
(\mathcal{F}(u)(x))^{2} & =\left[\Gamma(x) \int_{\mathbb{R}^{d}} c_{0}|x-y|^{2-d} V(y) u(y) \frac{d y}{\Gamma(x)}\right]^{2} \\
& \leq \Gamma(x) \int_{\mathbb{R}^{d}} c_{0}|x-y|^{2-d} V(y) u^{2}(y) d y .
\end{aligned}
$$

From this and (6.8) we deduce

$$
\int_{\mathbb{R}^{d}}(\mathcal{F}(u)(x))^{2} J(x) d x \leq c_{0} c_{1} \int_{\mathbb{R}^{d}} V(y) u^{2}(y)\left[\int_{\mathbb{R}^{d}} \min \left(|x|^{2-d}, 1\right)|x-y|^{2-d} J(x) d x\right] .
$$

If $V(y) \neq 0$ then $|y| \leq R_{0}$ for a suitable $R_{0}$. If

$$
I(y)=\int_{\mathbb{R}^{d}} \min \left(|x|^{2-d}, 1\right)|x-y|^{2-d} J(x) d x,
$$

then we have $\sup _{|y| \leq R_{0}} I(y)<\infty$ because

$$
\begin{aligned}
I(y) & \leq \int_{|x| \leq 2 R_{0}}+\int_{|x|>2 R_{0}} \min \left(|x|^{2-d}, 1\right)|x-y|^{2-d} J(x) d x \\
& \leq C \int_{|x-y| \leq 2 R_{0}}|x-y|^{2-d} d x+C \int_{|x|>2 R_{0}}|x|^{2-d}|x|^{2-d} J(x) d x<\infty
\end{aligned}
$$

by our assumption on $J$. As a result,

$$
\int_{\mathbb{R}^{d}}(\mathcal{F}(u)(x))^{2} J(x) d x \leq C \int_{\mathbb{R}^{d}} V(y) u^{2}(y) d y<\infty
$$

because $V$ is of compact support. This shows that $\mathcal{F}(u) \in \mathcal{H}$ whenever $u \in \mathcal{H}$.

Step 2. Observe that $\mathcal{H}$ is a Hilbert space with respect to the inner product

$$
\langle u, v\rangle=\int_{\mathbb{R}^{d}} u(x) v(x) J(x) d x .
$$

Note that if $u$ solves (6.1), then

$$
u+\beta(-\Delta)^{-1}((1+u) V)=0
$$


As a result,

$$
(i d+\beta \mathcal{F})(u)=g
$$

where $g(x)=-\beta \Gamma(x)$ with $\Gamma$ as in (6.7) and $i d$ means the identity transformation. The formulation (6.10) allows us to use some standard arguments to establish the existence of a solution of (6.1). Note that our assumption on $J$ implies that $\Gamma \in \mathcal{H}$ because of (6.8). Hence as a strategy for the existence, we show that the range of the operator $i d+\beta \mathcal{F}$ is the space $\mathcal{H}$. By the Fredholm Alternative Theorem, it suffices to show that the operator $\mathcal{F}$ is compact and that the operator $i d+\beta \mathcal{F}$ has a trivial kernel.

Step 3. In this step we verify the injectivity of the operator $i d+\beta \mathcal{F}$. Let us assume that for some $u \in \mathcal{H}, \beta \mathcal{F} u=-u$. From this we deduce $\Delta u=\beta u V$ weakly by standard arguments. Using Sobolev's inequalities and a bootstrap we can readily show that $u \in C^{2}$ because $V$ is continuous. As a result, $\Delta u=\beta u V$ in the classical sense. We also have

$$
u(x)=-c_{0} \beta \int_{\mathbb{R}^{d}} u(y)|x-y|^{2-d} V(y) d y .
$$

We now assert

$$
\begin{gathered}
\lim _{|x| \rightarrow \infty} u(x)|x|^{d-2}=-c_{0} \beta \int_{\mathbb{R}^{d}} V(y) u(y) d y, \\
\lim _{|x| \rightarrow \infty}|\nabla u(x)||x|^{d-1} \leq c_{0}(d-2) \beta \int_{\mathbb{R}^{d}} V(y)|u(y)| d y .
\end{gathered}
$$

The proof of (6.12) follows from

$$
\lim _{|x| \rightarrow \infty}|x|^{d-2}|x-y|^{2-d}=1
$$

for every $y$, and the elementary inequality

$$
|x|^{d-2}|x-y|^{2-d} \leq 2^{d-2}
$$

for $|x| \geq 2 R_{0}$ (because $|y| \leq R_{0}$ and $|x-y| \geq|x| / 2$ whenever $|x| \geq 2 R_{0}$ ). The proof of (6.13) is similar. We now choose $R>R_{0}$ and use $\Delta u=\beta V u$ to write

$$
\int_{|x| \leq R} u \Delta u d x=\beta \int_{|x| \leq R} V u^{2} d x .
$$

After an integration by parts we obtain

$$
-\int_{|x| \leq R}|\nabla u|^{2} d x+\int_{|x|=R} u \nabla u \cdot n d \sigma=\beta \int_{|x| \leq R} V u^{2} d x
$$

where $n=\frac{x}{|x|}$ is the normal vector and $d \sigma$ is the surface measure on $|x|=R$. From (6.12) $-(6.13)$ we can readily deduce

$$
\int_{|x|=R} u \nabla u \cdot n d \sigma=O\left(R^{2-d}\right)
$$

As a result,

$$
-\int_{\mathbb{R}^{d}}|\nabla u|^{2} d x=\beta \int_{\mathbb{R}^{d}} V u^{2} d x
$$


From this we deduce $\int_{\mathbb{R}^{d}}|\nabla u|^{2} d x=\int_{\mathbb{R}^{d}} V u^{2} d x=0$. This in turn implies $u \equiv 0$.

Step 4. In this step we verify the compactness of the operator $\mathcal{F}$. Set $\phi(x)=c_{0}|x|^{2-d}$ so that $\mathcal{F}(u)=(V u) * \phi$. We then have $\nabla \mathcal{F}(u)=(V u) * \nabla \phi$. Let $B_{R}$ denote the ball $\{x:|x| \leq R\}$. We then use the Young's inequality to write

$$
\begin{gathered}
\|\nabla \mathcal{F}(u)\|_{L^{2}\left(B_{R}\right)} \leq\|V u\|_{L^{2}\left(B_{R}\right)}\|\nabla \phi\|_{L^{1}\left(B_{R}\right)} \leq C R\|V u\|_{L^{2}\left(B_{R}\right)}, \\
\|\mathcal{F}(u)\|_{L^{2}\left(B_{R}\right)} \leq\|V u\|_{L^{2}\left(B_{R}\right)}\|\phi\|_{L^{1}\left(B_{R}\right)} \leq C R^{2}\|V u\|_{L^{2}\left(B_{R}\right)},
\end{gathered}
$$

for some constant $C$. Now if $\left\{u_{n}\right\}$ is bounded in $\mathcal{H}$, then by positivity of $J$ we deduce that $\sup _{n}\left\|u_{n}\right\|_{L^{2}\left(B_{R}\right)}<\infty$. This and (6.14)-(6.15) imply

$$
\sup _{n}\left(\left\|\mathcal{F}\left(u_{n}\right)\right\|_{L^{2}\left(B_{R}\right)}+\left\|\nabla \mathcal{F}\left(u_{n}\right)\right\|_{L^{2}\left(B_{R}\right)}\right)<\infty .
$$

From this and Rellich's theorem we learn that $\left\{\mathcal{F}\left(u_{n}\right)\right\}$ has a convergent subsequence in $L_{l o c}^{2}\left(\mathbb{R}^{d}\right)$. As in Step 1 we have

$$
\int_{|x| \geq l}\left(\mathcal{F}\left(u_{n}\right)(x)\right)^{2} J(x) d x \leq C\left[\int_{|x| \geq l}|x|^{4-2 d} J(x) d x\right] \cdot \int_{\mathbb{R}^{d}} V(y) u_{n}^{2}(y) d y,
$$

for $l \geq 2 R_{0}$. As a result,

$$
\lim _{l \rightarrow \infty} \sup _{n} \int_{|x| \geq l}\left(\mathcal{F}\left(u_{n}\right)(x)\right)^{2} J(x) d x=0 .
$$

This and the precompactness of $\left\{\mathcal{F}\left(u_{n}\right)\right\}$ in $L_{l o c}^{2}\left(\mathbb{R}^{d}\right)$ imply the precompactness of $\left\{\mathcal{F}\left(u_{n}\right)\right\}$ in $\mathcal{H}$. Step 5. So far we have shown the existence of a unique solution $u \in \mathcal{H}$ of $u+\beta \mathcal{F}(u)=g$. As in Step 3 we can readily show that $u$ is a weak solution of (6.1). Again we can use the Sobolev's inequality and a bootstrap to show that in fact $u \in C^{2}\left(\mathbb{R}^{d}\right)$ and satisfies (6.1) in the classical sense. Step 6. It remains to show that for the unique solution $u$ of (6.1), we have $-1 \leq u(x) \leq 0$ for every $x$. First take a smooth function $\varphi_{\delta}: \mathbb{R} \rightarrow(-\infty, 0]$ such that $\varphi_{\delta}^{\prime} \geq 0$ and

$$
\varphi_{\delta}(r)= \begin{cases}0 & r>-1 \\ 1+r & r<-1-\delta\end{cases}
$$

We then have

$$
-\int_{\mathbb{R}^{d}} \varphi_{\delta}^{\prime}(u)|\nabla u|^{2} d x=\int_{\mathbb{R}^{d}} \varphi_{\delta}(u) \Delta u d x=\beta \int_{\mathbb{R}^{d}} V(1+u) \varphi_{\delta}(u) d x
$$

by an integration by parts. The formula (6.17) is established with the aid of (6.12) as in Step 3 . Since the left-hand side of (6.17) is negative and $(1+u) \varphi_{\delta}(u) \geq 0$ we deduce

$$
\int_{\mathbb{R}^{d}} \varphi_{\delta}^{\prime}(u)|\nabla u|^{2} d x=\int_{\mathbb{R}^{d}} V(1+u) \varphi_{\delta}(u) d x=0 .
$$

We now send $\delta \rightarrow 0$ to deduce

$$
0=\int_{\mathbb{R}^{d}}|\nabla u|^{2} \mathbb{1}(1+u \leq 0) d x=\int_{\mathbb{R}^{d}} V(1+u)^{2} \mathbb{1}(1+u \leq 0) d x .
$$

As a result, on the set $A=\{x: 1+u(x)<0\}$ we have $\nabla u=0$. Hence $u$ is constant on each component $B$ of $A$. But this constant can only be -1 because on the boundary of $A$ we have 
$1+u=0$. This is impossible unless $A$ is empty and we deduce that $u \geq-1$ everywhere. Finally, from

$$
u(x)=c_{0} \int_{\mathbb{R}^{d}} V(y)(1+u(y))|x-y|^{2-d} d y,
$$

and $1+u \geq 0$ we deduce that $u \leq 0$.

Proof of Theorem 6.2 Let us write $u^{\beta}$ for the unique solution of (6.1). Note that by Theorem6.1,

$$
-1 \leq u^{\beta}(x) \leq 0
$$

for every $x$. Since $\Delta u^{\beta}=\beta\left(1+u^{\beta}\right) V$, we also have

$$
u^{\beta}(x)=-c_{0} \int_{\mathbb{R}^{d}}|x-y|^{2-d} \mu^{\beta}(d y) .
$$

where $\mu^{\beta}(d y)=\beta\left(1+u^{\beta}(y)\right) V(y) d y$. From this, (6.18) and (6.4) we learn

$$
F(\beta)=\int_{\mathbb{R}^{d}} \beta\left(1+u^{\beta}(y)\right) V(y) d y=\mu^{\beta}\left(\mathbb{R}^{d}\right) \leq \operatorname{Cap}\left(K_{0}\right) .
$$

It remains to show $\lim _{\beta \rightarrow \infty} F(\beta)=\operatorname{Cap}\left(K_{0}\right)$. By (6.20) the family $\left\{\mu^{\beta}\right\}$ is precompact with respect to the weak topology. Let $\mu$ be a limit point and put

$$
u(x)=-c_{0} \int_{\mathbb{R}^{d}}|x-y|^{2-d} \mu(d y) .
$$

Assume $\lim _{\beta^{\prime} \rightarrow \infty} \mu^{\beta^{\prime}}=\mu$ where $\left\{\mu^{\beta^{\prime}}\right\}$ is a subsequence of $\left\{\mu^{\beta}\right\}$. It is not hard to see

$$
\limsup _{\beta^{\prime} \rightarrow \infty} u^{\beta^{\prime}}(x) \leq u(x)
$$

by replacing the integrand in (6.21) and (6.19) with a bounded cutoff function and passing to the limit. Since $u^{\beta} \geq-1$, we deduce that $u \geq-1$. Also (6.21) implies that $u \leq 0$. Hence $-1 \leq u \leq 0$.

In fact it is well known that $\limsup _{\beta \rightarrow \infty} u^{\beta^{\prime}}(x)=u(x)$ except on a set of zero capacity. (See for example page 161 or the proof of Theorem 5.7.1 in [1].) Since $\Delta u^{\beta}=\mu^{\beta}, \Delta u=\mu$ and $\mu^{\beta} \Rightarrow \mu$, we can readily deduce that $u^{\beta} \rightarrow u$ weakly. As a result,

$$
\lim _{\beta \rightarrow \infty} \int_{\mathbb{R}^{d}}\left(1+u^{\beta}\right) V d x=\int_{\mathbb{R}^{d}}(1+u) V d x .
$$

Since $\beta \int_{\mathbb{R}^{d}}\left(1+u^{\beta}\right) V d x$ is bounded in $\beta$, we deduce

$$
\int_{\mathbb{R}^{d}}(1+u) V d x=0
$$

As a result, $u \equiv-1$ almost everywhere in $U_{0}=\{x: V(x) \neq 0\}$. Since $u(x)=\limsup _{y \rightarrow x} u(y)$ except for a set of zero capacity, we learn $u \equiv-1$ in $U_{0}$ except for a set of zero capacity. (See, for example, Theorem 3.7.5 of [1].) Let $\nu$ denote the equilibrium measure of $K_{0}$. That is, $\operatorname{supp}(\nu) \subset K_{0}$, $\nu\left(K_{0}\right)=C a p\left(K_{0}\right)$, and that the function $v(x)=c_{0} \int_{\mathbb{R}^{d}}|x-y|^{2-d} \nu(d y)$ satisfies $v \equiv 1$ in $K_{0}$ except 
for a set of zero capacity. Also, since $u$ is finite everywhere, we learn that $\mu(A)=0$ whenever $\operatorname{Cap}(A)=0$. The same is also true for $\nu$. (See for example Theorem 5.1 .9 of [1].) As a result,

$$
\mu\left(K_{0}\right)=\int_{\mathbb{R}^{d}} v d \mu=c_{0} \int_{\mathbb{R}^{2 d}}|x-y|^{2-d} \nu(d y) \mu(d x)=-\int_{\mathbb{R}^{d}} u(y) \nu(d y) .
$$

But $\nu(A)=0$ if $\operatorname{Cap}(A)=0$ and $-u \equiv 1$ on $K_{0}$ except for a set of zero capacity. This and (6.22) imply

$$
\mu\left(K_{0}\right)=\nu\left(K_{0}\right)=\operatorname{Cap}\left(K_{0}\right)
$$

\section{REFERENCES}

[1] David H. Armitage and Stephen J. Gardiner. Classical potential theory. Springer, 2001.

[2] Schlein B. Erdos, L. and H.T. Yau. Derivation of the gross-pitaevskii equation for the dynamics of bose-einstein condensate. www.arxiv.org/math-ph/0410005, 3:3, 3 .

[3] S. Großkinsky, C. Klingenberg, and K. Oelschläger. A rigorous derivation of Smoluchowski's equation in the moderate limit. Stochastic Anal. Appl., 22(1):113-141, 2004.

[4] Alan Hammond and Fraydoun Rezakhanlou. Moment bounds for the Smoluchowski equation and their consequences. Comm. Math. Phys., 276(3):645-670, 2007.

[5] O.E. III Lanford. Time evolution of large classical systems. Lecture Notes in Physics, 38:1-111, 1975.

[6] Reinhard Lang and Xuan-Xanh Nyugen. Smoluchowski's theory of coagulation in colloids holds rigorously in the Boltzmann-Grad limit. Z. Wahrsch. Verw. Gebiete, 54:227-280, 1980.

[7] J. R. Norris. Cluster coagulation. Comm. Math. Phys., 209(2):407-435, 2000.

[8] M Smoluchowski. Drei vortrage uber diffusion, Brown'sche molekularbewegung und koagulation von kolloidteilchen. Phys. Z. XVII, pages 557-571,585-599, 1916.

[9] A.-S. Sznitman. Propagation of chaos for a system of annihilating Brownian spheres. Comm. Pure Appl. Math., 40(6):663-690, 1987.

[10] Dariusz Wrzosek. Existence of solutions for the discrete coagulation-fragmentation model with diffusion. Topol. Methods Nonlinear Anal., 9(2):279-296, 1997.

[11] Dariusz Wrzosek. Mass-conserving solutions to the discrete coagulation-fragmentation model with diffusion. Nonlinear Anal., 49(3, Ser. A: Theory Methods):297-314, 2002. 